\title{
Performance Analysis of Two-Dimensional Maximum Likelihood Direction-of-Arrival Estimation Algorithm Using the UCA
}

\author{
Yun-Seong Cho, Jeong-Min Seo, and Joon-Ho Lee \\ Department of Information \& Communication Engineering, Sejong University, 98 Kunja-dong, Kwangjin-ku, \\ Seoul 143-747, Republic of Korea
}

Correspondence should be addressed to Joon-Ho Lee; joonhlee@sejong.ac.kr

Received 2 June 2016; Revised 23 August 2016; Accepted 7 September 2016; Published 9 February 2017

Academic Editor: Sergiy A. Vorobyov

Copyright (C) 2017 Yun-Seong Cho et al. This is an open access article distributed under the Creative Commons Attribution License, which permits unrestricted use, distribution, and reproduction in any medium, provided the original work is properly cited.

\begin{abstract}
We address the performance analysis of the maximum likelihood (ML) direction-of-arrival (DOA) estimation algorithm in the case of azimuth/elevation estimation of two incident signals using the uniform circular array (UCA). Based on the Taylor series expansion and approximation, we get explicit expressions of the root mean square errors (RMSEs) of the azimuths and elevations. The validity of the derived expressions is shown by comparing the analytic results with the simulation results. The derivation in this paper is further verified by illustrating the consistency of the analytic results with the Cramer-Rao lower bound (CRLB).
\end{abstract}

\section{Introduction}

There have been many studies on the direction-of-arrival (DOA) estimation [1-5]. Our concern in this paper is the performance analysis of the maximum likelihood- (ML-) based DOA estimation algorithm.

Many studies have been conducted on asymptotic performance analysis of maximum likelihood DOA estimation algorithm [6-8]. The shortcoming of the asymptotic performance analysis is that it is only applicable to high SNR region since it is based on approximation which is valid only for small amount of noise. To circumvent this problem, many studies on nonasymptotic performance analysis have been conducted for performance analysis which is valid for low SNR as well as high SNR.

In [9-12], the authors dealt with threshold performance analysis for single incident signal. On the other hand, our scheme presented in this paper is applicable to multiple incident signal as well as single incident signal.

In this paper, we address the performance analysis of the ML algorithm for the estimation of the DOA of incident signals. Our interest is in the case of multiple incident signals. The ML algorithm exploits the fact that, in the absence of the noise, the incident signal on the array elements can be expressed as a linear combination of the array steering vectors corresponding to the true incident angles.

Based on this observation, in the ML algorithm, the estimate of the DOA is obtained from the angles whose steering vectors can span the subspace to which the signal on the array elements belongs.

In practical situation, the noise is inevitable. Therefore, the noisy incident signal on the array elements cannot be expressed as a linear combination of the array steering vectors associated with true incident angles, which accounts for why estimation error occurs for noisy array response.

To quantify the bias of the estimate due to the noise on the antenna elements, we adopt the Taylor series approximation around the true incident angle. We derive the closed-form expression of the biases of the estimates, where different estimates are due to some approximations. For one of the many estimates, we derive the closed-form expression of the MSE of the estimate.

In Figure 1, the mathematical and statistical theories used for the performance analysis of the ML algorithm are enumerated.

Novelty of the proposed method over the existing methods is as follows. 


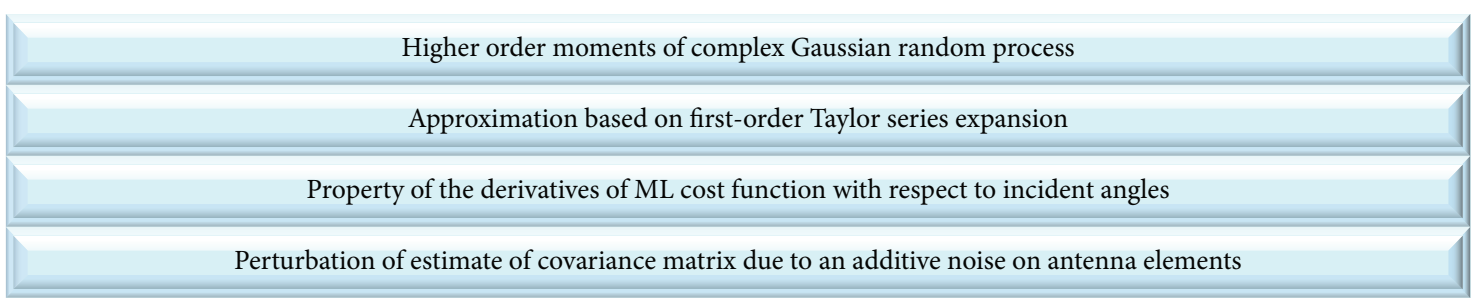

FIGURE 1: Mathematical/statistical foundation of the proposed analytical performance analysis of ML algorithm.

Performance Analysis for Multiple Incident Signals and Simultaneous Estimation of Azimuth/Elevation. In [9-11], the authors made performance analysis of ML DOA algorithm for single incident signals. On the other hand, the scheme presented in this paper handles multiple incident signals as well as single incident signal. In addition, the studies presented in [9-11] consider the estimation of azimuth, not azimuth and elevation. Note that, in this paper, we consider the estimation of azimuth and elevation of multiple incident signals.

Derivation of Explicit Expressions of Bias and MSE, Not the Lower Bound of MSE. In [13], the authors derived the CRLB of the DOA algorithm, which is a lower bound of the variance of the DOA algorithm. Note that the CRLB is the lower bound of the variance, not the variance itself of the DOA algorithm. In this paper, explicit expression of the bias of the estimate and explicit expression of the MSE itself, not the CRLB, have been derived.

Intuition on the Effect of Each Approximation on the Estimation Accuracy. In this paper, we obtain, for $c=1,2$, the expressions of $\left[\widehat{\theta}_{c}, \widehat{\phi}_{c}\right],\left[\widehat{\theta}_{c}^{(u=1)}, \widehat{\phi}_{c}^{(u=1)}\right]$, and $\left[\widehat{\theta}_{c}^{(u=1, v)}\right.$, $\left.\widehat{\phi}_{c}^{(u=1, v)}\right]$. The superscripts $u$ and $v$ denote $U$ approximation and $V$ approximation, respectively. Therefore, $\left[\widehat{\theta}_{c}, \widehat{\phi}_{c}\right],\left[\widehat{\theta}_{c}^{(u=1)}\right.$, $\left.\hat{\phi}_{c}^{(u=1)}\right]$, and $\left[\hat{\theta}_{c}^{(u=1, v)}, \hat{\phi}_{c}^{(u=1, v)}\right]$ are the estimates with no approximation, $U$ approximation, and $U / V$ approximations, respectively. By comparing these three values, we can intuitively recognize which approximation of $U$ approximation and $V$ approximation results in larger error. The importance of this observation is that the proposed scheme gives us an intuition on which approximation of all the approximations results in large approximation error. The approach presented in [14] does not show any intuition on which approximation results in large approximation error, since in [14] the authors only explicitly consider Taylor series expansion for general problem. Since the approach in [14] is a general approach applicable to many estimation problems, it does not give explicit expressions specific to DOA estimation problem. Since the scheme presented in this paper only considers the estimation of DOA estimation, rather than general estimation problem, the expressions presented in this paper are more explicit than the results presented in [14].

Explicit Expressions of Various Estimates for Each Step of Successive Approximations. To the best of our knowledge, there has been no study where explicit expressions of successive estimates have been derived [6-13]. Successive estimates imply various estimates obtained as each approximation has been successively applied. In [14], no explicit expression of estimate for each step of successive approximations has been presented. Although the study presented in [14] deals with the estimation of vector parameter as well as scalar parameter, the results in [14] for vector parameter estimation, equations (41) and (42) in [14], are quite implicit, rather than explicit, and it is very difficult to get intuition on how the bias and the covariance matrix are dependent on the amount of an additive noise.

Search-Free Approximate ML DOA Estimation and Derivation of Closed-Form Estimation Error. For getting $\widehat{\theta}_{c}$ and $\widehat{\phi}_{c}$ in (13) for $c=1,2$, four-dimensional search with respect to $\theta_{1}, \theta_{2}$, $\phi_{1}$, and $\phi_{2}$ has to be performed. In general, for estimation of $d$ incident signals, $2 d$ dimensional search has to be performed for simultaneous estimation of $\left[\theta_{1}, \phi_{1}\right], \ldots,\left[\theta_{d}, \phi_{d}\right]$, which is computationally very intensive. In addition, in practical implementation of (13), the estimates are highly dependent on the search range and search step, which implies that the estimates in (13) may be wrong if search step and search range are not properly chosen. In summary, the original ML estimate $\left[\widehat{\theta}_{c}, \widehat{\phi}_{c}\right]$ obtained from implementation of (13) has two demerits of computational cost and dependence on search step and search range.

On the other hand, $\left[\delta \theta_{c}^{(u=1)}, \delta \phi_{c}^{(u=1)}\right]$ and $\left[\delta \theta_{c}^{(u=1, v)}\right.$, $\left.\delta \phi_{c}^{(u=1, v)}\right]$ are obtained from (42) and (45), respectively. Note that $\left[\delta \theta_{c}^{(u=1)}, \delta \phi_{c}^{(u=1)}\right]$ and $\left[\delta \theta_{c}^{(u=1, v)}, \delta \phi_{c}^{(u=1, v)}\right]$ are obtained in closed-form and that evaluation of $\left[\delta \theta_{c}^{(u=1)}, \delta \phi_{c}^{(u=1)}\right]$ and $\left[\delta \theta_{c}^{(u=1, v)}, \delta \phi_{c}^{(u=1, v)}\right]$ is search-free, which implies that evaluation of $\left[\delta \theta_{c}^{(u=1)}, \delta \phi_{c}^{(u=1)}\right]$ and $\left[\delta \theta_{c}^{(u=1, v)}, \delta \phi_{c}^{(u=1, v)}\right]$ is much less computationally intensive than evaluation of $\left[\theta_{c}^{(u=1)}, \phi_{c}^{(u=1)}\right]$. In addition, since evaluation of $\left[\delta \theta_{c}^{(u=1)}, \delta \phi_{c}^{(u=1)}\right]$ and $\left[\delta \theta_{c}^{(u=1, v)}, \delta \phi_{c}^{(u=1, v)}\right]$ is search-free, $\left[\delta \theta_{c}^{(u=1)}, \delta \phi_{c}^{(u=1)}\right]$ and $\left[\delta \theta_{c}^{(u=1, v)}, \delta \phi_{c}^{(u=1, v)}\right]$ are not dependent on search parameters such as search step and search range.

$$
\left[\delta \theta_{c}^{(u=1)}, \delta \phi_{c}^{(u=1)}\right] \text { and }\left[\delta \theta_{c}^{(u=1, v)}, \delta \phi_{c}^{(u=1, v)}\right] \text { in (42) and (45) }
$$
can be regarded as estimation errors for approximate ML DOA algorithm. $\left[\delta \theta_{c}^{(u=1)}, \delta \phi_{c}^{(u=1)}\right]$ and $\left[\delta \theta_{c}^{(u=1, v)}, \delta \phi_{c}^{(u=1, v)}\right]$ can be obtained in closed-form, although $\left[\delta \theta_{c}^{(u=1)}, \delta \phi_{c}^{(u=1)}\right]$ and $\left[\delta \theta_{c}^{(u=1, v)}, \delta \phi_{c}^{(u=1, v)}\right]$ are less accurate than $\left[\delta \theta_{c}, \delta \phi_{c}\right]$ in the sense that $U$ approximation is applied in getting 
$\left[\delta \theta_{c}^{(u=1)}, \delta \phi_{c}^{(u=1)}\right]$ and that $U / V$ approximations are applied in getting $\left[\delta \theta_{c}^{(u=1, v)}, \delta \phi_{c}^{(u=1, v)}\right]$.

Derivation of Analytic Expression of Closed-Form MSE of $\left[\delta \theta_{c}^{(u=1, v)}, \delta \phi_{c}^{(u=1, v)}\right]$. Many evaluations of (42) and (45) are required for getting empirical performance analysis. Single evaluation of (42) and (45) is not computationally intensive since they are search-free closed-form solutions. Note that main computational cost of evaluating (42) and (45) is inversion of $2 \times 2$ matrices, which is not computationally intensive at all. For estimation of $d$ incident signals, $d \times d$ matrices have to be inverted to implement (42) and (45). But for empirical performance analysis based on Monte-Carlo simulation, (42) and (45) have to be evaluated many times. Note that the number of repetitions should be large for getting reliable empirical performance analysis, which implies that the empirical performance analysis based on many evaluations of (42) and (45) is computationally intensive. In this paper, it has been shown that empirical performance of $\left[\delta \theta_{c}^{(u=1, v)}, \delta \phi_{c}^{(u=1, v)}\right]$ can be obtained from (47), without many evaluations of (45). Note that single evaluation of (47) results in analytic performance analysis, which should be equivalent to empirical performance based on many repetitions of (45). Therefore, although it is not necessary to repeatedly evaluate (45) to get empirical MSE, (45) is evaluated many times for validating the derived expression (47). Also, note that since analytic expression of the MSE of $\left[\theta_{c}^{(u=1)}, \phi_{c}^{(u=1)}\right]$ is not available, the MSE of $\left[\theta_{c}^{(u=1)}, \phi_{c}^{(u=1)}\right]$ has to be empirically obtained via many evaluations of (42) with different realizations of additive noise for each evaluation.

In $\hat{\theta}_{c}^{(u=1)}$ and $\widehat{\theta}_{c}^{(u=1, v)}$, the superscripts $u$ and $v$ denote $U$ approximation and $V$ approximation, respectively. On the other hand, in [14], no explicit expression of MSE is presented, and only the implicit expression of final estimate is presented. Note that, in this paper, explicit expressions of all the estimates associated with each successive approximation have been presented.

\section{ML DOA Algorithm}

For simplicity, we present performance analysis for two incident signals on the UCA in this paper. But, extension to more incident signals and general planar array structure is straightforward. Let $\Theta, \widehat{\Theta}, \Theta^{(0)}$, and $\delta \Theta$ represent

$$
\begin{aligned}
\Theta & =\left(\theta_{1}, \theta_{2}, \phi_{1}, \phi_{2}\right), \\
\widehat{\Theta} & =\left(\widehat{\theta}_{1}, \widehat{\theta}_{2}, \widehat{\phi}_{1}, \widehat{\phi}_{2}\right), \\
\Theta^{(0)} & =\left(\theta_{1}^{(0)}, \theta_{2}^{(0)}, \phi_{1}^{(0)}, \phi_{2}^{(0)}\right), \\
\delta \Theta & =\left(\delta \theta_{1}, \delta \theta_{2}, \delta \phi_{1}, \delta \phi_{2}\right) .
\end{aligned}
$$

The number of the antenna elements is denoted by $M$. When there is no noise, the received signals on the antenna elements are expressed as

$$
\mathbf{x}\left(t_{n}\right)=\mathbf{A}(\Theta) \mathbf{s}\left(t_{n}\right),
$$

where $\mathbf{s}\left(t_{n}\right)$ is given by

$$
\mathbf{s}\left(t_{n}\right)=\left[s_{1}\left(t_{n}\right) s_{2}\left(t_{n}\right)\right]^{T}
$$

Array matrix in (2) is written as

$$
\mathbf{A}(\Theta)=\left[\mathbf{a}\left(\theta_{1}, \phi_{1}\right) \mathbf{a}\left(\theta_{2}, \phi_{2}\right)\right]
$$

where array vector is defined as

$$
\begin{aligned}
& \mathbf{a}\left(\theta_{n}, \phi_{n}\right) \\
& \quad=\left[\begin{array}{llll}
a_{1}\left(\theta_{n}, \phi_{n}\right) & a_{2}\left(\theta_{n}, \phi_{n}\right) & \cdots & a_{M}\left(\theta_{n}, \phi_{n}\right)
\end{array}\right]^{T}, \\
& a_{m}\left(\theta_{n}, \phi_{n}\right)=\exp \left[j \psi_{m}\left(\theta_{n}, \phi_{n}\right)\right], \\
& \psi_{m}\left(\theta_{n}, \phi_{n}\right)=\frac{2 \pi r}{\lambda} \cos \left(\theta_{n}-\frac{2 \pi(m-1)}{M}\right) \cos \phi_{n} .
\end{aligned}
$$

The corresponding expression for the noisy signals is

$$
\mathbf{x}^{\prime}\left(t_{n}\right)=\mathbf{A}(\Theta) \mathbf{s}\left(t_{n}\right)+\mathbf{n}\left(t_{n}\right)
$$

where $\theta$ and $\phi$ denote the azimuth and the elevation, respectively.

Expression (7) for a general planar array with $L \times M$ elements can be written as

$$
\begin{aligned}
& \psi_{l, m}\left(\theta_{n}, \phi_{n}\right)=\frac{2 \pi}{\lambda}\left((l-1) \Delta x \sin \theta_{n} \cos \phi_{n}\right. \\
& \left.+(m-1) \Delta y \cos \theta_{n} \cos \phi_{n}\right), \\
& \qquad=1 \cdots L, m=1 \cdots M,
\end{aligned}
$$

where $\Delta x$ and $\Delta y$ denote the element spacing in the $x$ direction and $y$-direction, respectively, and $l$ and $m$ denote the index in the $x$-direction and $y$-direction, respectively.

Also, (8) for general planar array is written as

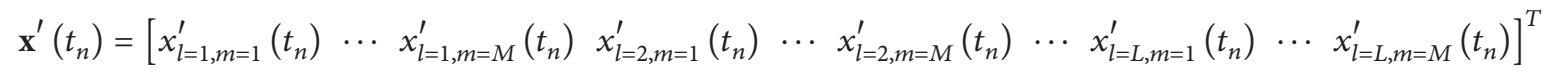




$$
\left[\begin{array}{ccc}
a_{l=1, m=1}\left(\theta_{1}, \phi_{1}\right) & \cdots & a_{l=1, m=1}\left(\theta_{N}, \phi_{N}\right) \\
\vdots & & \vdots \\
a_{l=1, m=M}\left(\theta_{1}, \phi_{1}\right) & \cdots & a_{l=1, m=M}\left(\theta_{N}, \phi_{N}\right) \\
a_{l=2, m=1}\left(\theta_{1}, \phi_{1}\right) & \cdots & a_{l=2, m=1}\left(\theta_{N}, \phi_{N}\right) \\
\vdots & & \vdots \\
a_{l=2, m=M}\left(\theta_{1}, \phi_{1}\right) & \cdots & a_{l=2, m=M}\left(\theta_{N}, \phi_{N}\right) \\
\vdots & & \vdots \\
a_{l=L, m=1}\left(\theta_{1}, \phi_{1}\right) & \cdots & a_{l=L, m=1}\left(\theta_{N}, \phi_{N}\right) \\
\vdots & \vdots \\
a_{l=L, m=M}\left(\theta_{1}, \phi_{1}\right) & \cdots & a_{l=L, m=M}\left(\theta_{N}, \phi_{N}\right)
\end{array}\right]\left[\begin{array}{c}
s_{1}\left(t_{n}\right) \\
\vdots \\
s_{N}\left(t_{n}\right)
\end{array}\right]+\left[\begin{array}{c}
n_{l=1, m=1}\left(t_{n}\right) \\
\vdots \\
n_{l=1, m=M}\left(t_{n}\right) \\
n_{l=2, m=1}\left(t_{n}\right) \\
\vdots \\
n_{l=2, m=M}\left(t_{n}\right) \\
\vdots \\
n_{l=L, m=1}\left(t_{n}\right) \\
\vdots \\
n_{l=L, m=M}\left(t_{n}\right)
\end{array}\right] .
$$

We have explicitly shown how (7) and (8) should be modified for a two-dimensional planar array. Note that, for an arbitrary array structure, (7) and (8) are modified appropriately to apply the proposed algorithm to the specific array structure. (7) for an arbitrary array structure can be easily obtained from the phase difference between antenna elements, given the azimuth and the elevation of the $n$th incident signal. Note that the phase difference is both dependent on the array geometry for a specific array structure and the direction of the incident signal.

The projection matrix onto the column space of $\mathbf{A}$ can be expressed as [15]. It is assumed that the entries of the Gaussian vector are independent and identically distributed Gaussian random variables with zero-mean and the same variance. Note that the noise is complex-valued and that the real part and the imaginary part of the noise are independent Gaussian random variables with zero-mean. The variance of the real part is denoted by $\sigma^{2} / 2$, which is equal to the variance of the imaginary part, $\sigma^{2} / 2$ :

$$
\mathbf{P}_{\mathbf{A}}(\Theta)=\mathbf{A}(\Theta)\left(\mathbf{A}^{H}(\Theta) \mathbf{A}(\Theta)\right)^{-1} \mathbf{A}^{H}(\Theta) .
$$

Note that, in this paper, the formulation is for the case where there are two incident signals. But, it can be easily extended to the case of more incident signals. Using the maximum likelihood (ML) algorithm, the estimates are given by [16]

$$
\widehat{\Theta}=\arg \max _{\Theta} \operatorname{tr}\left(\mathbf{P}_{\mathbf{A}}(\Theta) \widehat{\mathbf{R}}^{\prime}\right)
$$

More specifically, (12) for $d=2$ is written as

$$
\left[\widehat{\theta}_{1}, \widehat{\theta}_{2}, \widehat{\phi}_{1}, \widehat{\phi}_{2}\right]=\arg \max _{\theta_{1}, \theta_{2}, \phi_{1}, \phi_{2}} \operatorname{tr}\left(\mathbf{P}_{\mathbf{A}}\left(\theta_{1}, \theta_{2}, \phi_{1}, \phi_{2}\right) \mathbf{R}\right) .
$$

For notational simplicity, $\mathbf{P}(\Theta)$ is used to denote $\mathbf{P}_{\mathbf{A}}(\Theta)$ :

$$
\mathbf{P}(\Theta) \equiv \mathbf{P}_{\mathbf{A}}(\Theta)
$$

$\widehat{\mathbf{R}}^{\prime}$ is defined from

$$
\widehat{\mathbf{R}}^{\prime}=\frac{1}{T} \sum_{n=1}^{T} \mathbf{x}^{\prime}\left(t_{n}\right) \mathbf{x}^{\prime H}\left(t_{n}\right) .
$$

The explicit expression of the entries of the matrix $\left(\mathbf{A}^{H}(\Theta) \mathbf{A}(\Theta)\right)^{-1}$ in (11) is given by

$$
\mathbf{A}^{H}(\Theta) \mathbf{A}(\Theta)=\left[\begin{array}{cc}
M & \left(\mathbf{A}^{H} \mathbf{A}\right)_{12}(\Theta) \\
\left(\left(\mathbf{A}^{H} \mathbf{A}\right)_{12}(\Theta)\right)^{*} & M
\end{array}\right] .
$$

Using (4) and (5), $\left(\mathbf{A}^{H} \mathbf{A}\right)_{12}(\Theta)$ in (16) is given by

$$
\mathrm{Q}(\Theta) \equiv\left(\mathbf{A}^{H} \mathbf{A}\right)_{12}(\Theta)=\sum_{k=1}^{M}\left(a_{k}\left(\theta_{1}, \phi_{1}\right)\right)^{*} a_{k}\left(\theta_{2}, \phi_{2}\right) \text {. }
$$

Let $a_{m, n}$ represent $a_{m}\left(\theta_{n}, \phi_{n}\right)$ :

$$
a_{m, n} \equiv a_{m}\left(\theta_{n}, \phi_{n}\right) \text {. }
$$

Using (16) and (17), $\left(\mathbf{A}^{H}(\Theta) \mathbf{A}(\Theta)\right)^{-1}$ can be expressed as

$$
\begin{aligned}
\left(\mathbf{A}^{H}(\Theta) \mathbf{A}(\Theta)\right)^{-1} & =\frac{1}{D(\Theta)}\left[\begin{array}{cc}
M & -Q(\Theta) \\
-(Q(\Theta))^{*} & M
\end{array}\right] \\
D(\Theta) & \equiv \operatorname{det}\left(\mathbf{A}^{H}(\Theta) \mathbf{A}(\Theta)\right) \\
& =M^{2}-|Q(\Theta)|^{2} .
\end{aligned}
$$

Using (17), $|Q(\Theta)|^{2}$ can be written as

$$
|Q(\Theta)|^{2}=\sum_{k=1}^{M} \sum_{k^{\prime}=1}^{M}\left(a_{k, 1}\right)^{*} a_{k, 2} a_{k, 1}\left(a_{k, 2}\right)^{*}
$$

The explicit expression of the entries of the matrix $\mathbf{P}(\Theta)$ in (11) is given by

$$
P_{k l}(\Theta)=\frac{S_{k l}(\Theta)}{D(\Theta)} .
$$


Using (16)-(21), $S_{k l}(\Theta)$ in (22) is defined as

$$
\begin{aligned}
S_{k l}(\Theta) \equiv & \left(a_{l, 1}\right)^{*}\left(a_{k, 1} M-a_{k, 2} Q(\Theta)\right) \\
& +\left(a_{l, 2}\right)^{*}\left(a_{k, 2} M-a_{k, 1} Q(\Theta)\right) .
\end{aligned}
$$

Note that $P_{k l}(\Theta)$ denotes the entry at the $k$ th row and the lth column of $\mathbf{P}(\Theta)$. The explicit expression of the entries of the matrix $\operatorname{tr}\left(\mathbf{P}(\Theta) \widehat{\mathbf{R}}^{\prime}\right)$ in (12) is given by

$$
\begin{aligned}
& \operatorname{tr}\left(\mathbf{P}(\Theta) \widehat{\mathbf{R}}^{\prime}\right) \\
& =\operatorname{tr}\left(\left[\begin{array}{ccc}
P_{11}(\Theta) & \cdots & P_{1 M}(\Theta) \\
\vdots & \ddots & \vdots \\
P_{M 1}(\Theta) & \cdots & P_{M M}(\Theta)
\end{array}\right]\left[\begin{array}{ccc}
\widehat{R}_{11}^{\prime} & \cdots & \widehat{R}_{1 M}^{\prime} \\
\vdots & \ddots & \vdots \\
\widehat{R}_{M 1}^{\prime} & \cdots & \widehat{R}_{M M}^{\prime}
\end{array}\right]\right) \\
& =\sum_{k=1}^{M} \sum_{l=1}^{M} P_{k l}(\Theta) \widehat{R}_{l k}^{\prime} .
\end{aligned}
$$

Using (24) in (12), we get $\widehat{\Theta}$. The biases of the estimates are defined as

$$
\begin{aligned}
& \delta \theta_{1}=\widehat{\theta}_{1}-\theta_{1}^{(0)}, \\
& \delta \theta_{2}=\widehat{\theta}_{2}-\theta_{2}^{(0)}, \\
& \delta \phi_{1}=\widehat{\phi}_{1}-\phi_{1}^{(0)}, \\
& \delta \phi_{2}=\widehat{\phi}_{2}-\phi_{2}^{(0)} .
\end{aligned}
$$

\section{Closed-Form Expression of Estimation Error}

Since $\theta_{1}, \theta_{2}, \phi_{1}$, and $\phi_{2}$ should be the maximizing arguments of $\operatorname{tr}\left(\mathbf{P}(\Theta) \widehat{\mathbf{R}}^{\prime}\right)$, the first-order derivative of $\operatorname{tr}\left(\mathbf{P}(\Theta) \widehat{\mathbf{R}}^{\prime}\right)$ with respect to each argument should be zero at $\Theta=\widehat{\Theta}$

$$
\begin{gathered}
\frac{\partial}{\partial \theta_{1}} \operatorname{tr}\left(\left.\mathbf{P}(\Theta) \widehat{\mathbf{R}}^{\prime}\right|_{\Theta=\widehat{\Theta}}\right) \\
=\sum_{k=1}^{M} \sum_{l=1}^{M}\left(\left.\frac{\partial}{\partial \theta_{1}} P_{k l}(\Theta)\right|_{\Theta=\widehat{\Theta}}\right) \widehat{R}_{l k}^{\prime} \\
=\sum_{k=1}^{M} \sum_{l=1}^{M}\left(\left.P_{k l, \theta_{1}}(\Theta)\right|_{\Theta=\widehat{\Theta}}\right) \widehat{R}_{l k}^{\prime}=0, \\
\frac{\partial}{\partial \theta_{2}} \operatorname{tr}\left(\left.\mathbf{P}(\Theta) \widehat{\mathbf{R}}^{\prime}\right|_{\Theta=\widehat{\Theta}}\right) \\
=\sum_{k=1}^{M} \sum_{l=1}^{M}\left(\left.\frac{\partial}{\partial \theta_{2}} P_{k l}(\Theta)\right|_{\Theta=\widehat{\Theta}}\right) \widehat{R}_{l k}^{\prime} \\
=\sum_{k=1}^{M} \sum_{l=1}^{M}\left(\left.P_{k l, \theta_{2}}(\Theta)\right|_{\Theta=\widehat{\Theta}}\right) \widehat{R}_{l k}^{\prime}=0,
\end{gathered}
$$

$$
\begin{gathered}
\frac{\partial}{\partial \phi_{1}} \operatorname{tr}\left(\left.\mathbf{P}(\Theta) \widehat{\mathbf{R}}^{\prime}\right|_{\Theta=\widehat{\Theta}}\right) \\
=\sum_{k=1}^{M} \sum_{l=1}^{M}\left(\left.\frac{\partial}{\partial \phi_{1}} P_{k l}(\Theta)\right|_{\Theta=\widehat{\Theta}}\right) \widehat{R}_{l k}^{\prime} \\
=\sum_{k=1}^{M} \sum_{l=1}^{M}\left(\left.P_{k l, \phi_{1}}(\Theta)\right|_{\Theta=\widehat{\Theta}}\right) \widehat{R}_{l k}^{\prime}=0, \\
\frac{\partial}{\partial \phi_{2}} \operatorname{tr}\left(\left.\mathbf{P}(\Theta) \widehat{\mathbf{R}}^{\prime}\right|_{\Theta=\widehat{\Theta}}\right) \\
=\sum_{k=1}^{M} \sum_{l=1}^{M}\left(\left.\frac{\partial}{\partial \phi_{2}} P_{k l}(\Theta)\right|_{\Theta=\widehat{\Theta}}\right) \widehat{R}_{l k}^{\prime} \\
=\sum_{k=1}^{M} \sum_{l=1}^{M}\left(\left.P_{k l, \phi_{2}}(\Theta)\right|_{\Theta=\widehat{\Theta}}\right) \widehat{R}_{l k}^{\prime}=0,
\end{gathered}
$$

where the first-order partial derivatives of $P_{k l}(\Theta)$ in (26) with respect to each argument are expressed as, using (22),

$$
\begin{aligned}
& P_{k l, \theta_{1}}(\Theta) \equiv \frac{\partial}{\partial \theta_{1}} P_{k l}(\Theta) \\
& =\frac{\left(\left(\partial / \partial \theta_{1}\right) S_{k l}(\Theta)\right) D(\Theta)-S_{k l}(\Theta)\left(\left(\partial / \partial \theta_{1}\right) D(\Theta)\right)}{(D(\Theta))^{2}} \\
& P_{k l, \theta_{2}}(\Theta) \equiv \frac{\partial}{\partial \theta_{2}} P_{k l}(\Theta) \\
& =\frac{\left(\left(\partial / \partial \theta_{2}\right) S_{k l}(\Theta)\right) D(\Theta)-S_{k l}(\Theta)\left(\left(\partial / \partial \theta_{2}\right) D(\Theta)\right)}{(D(\Theta))^{2}} \\
& P_{k l, \phi_{1}}(\Theta) \equiv \frac{\partial}{\partial \phi_{1}} P_{k l}(\Theta) \\
& =\frac{\left(\left(\partial / \partial \phi_{1}\right) S_{k l}(\Theta)\right) D(\Theta)-S_{k l}(\Theta)\left(\left(\partial / \partial \phi_{1}\right) D(\Theta)\right)}{(D(\Theta))^{2}} \\
& P_{k l, \phi_{2}}(\Theta) \equiv \frac{\partial}{\partial \phi_{2}} P_{k l}(\Theta) \\
& =\frac{\left(\left(\partial / \partial \phi_{2}\right) S_{k l}(\Theta)\right) D(\Theta)-S_{k l}(\Theta)\left(\left(\partial / \partial \phi_{2}\right) D(\Theta)\right)}{(D(\Theta))^{2}} .
\end{aligned}
$$

The first-order partial derivatives of $D(\Theta)$ in $(20)$ and $S_{k l}(\Theta)$ in (23) with respect to each argument in (27) are expressed in the Appendices A and B. Denominator of equation (27) can be written as

$$
\begin{aligned}
(D(\Theta))^{2} & =D(\Theta) D(\Theta) \\
& =M^{4}-2 M^{2}|Q(\Theta)|^{2}+|Q(\Theta)|^{4} .
\end{aligned}
$$

Using (21), $|Q(\Theta)|^{4}$ is defined as

$$
\begin{aligned}
& |Q(\Theta)|^{4}=|Q(\Theta)|^{2}|Q(\Theta)|^{2} \\
& =\sum_{k=1}^{M} \sum_{k^{\prime}=1}^{M} \sum_{q=1}^{M} \sum_{q^{\prime}=1}^{M}\left(a_{k, 1}\right)^{*} a_{k, 2} a_{k^{\prime}, 1}\left(a_{k^{\prime}, 2}\right)^{*}\left(a_{q, 1}\right)^{*} a_{q, 2} a_{q^{\prime}, 1}\left(a_{q^{\prime}, 2}\right)^{*} .
\end{aligned}
$$


We define $\widehat{\mathbf{R}}$ in (26) as

$$
\widehat{\mathbf{R}}=\frac{1}{T} \sum_{n=1}^{T} \mathbf{x}\left(t_{n}\right) \mathbf{x}^{H}\left(t_{n}\right),
$$

where $\mathbf{x}\left(t_{n}\right)$ is given in (2). Let $\delta \mathbf{R}$ represent the difference between $\widehat{\mathbf{R}}^{\prime}$ and $\widehat{\mathbf{R}}$ :

$$
\begin{gathered}
\delta \mathbf{R}=\widehat{\mathbf{R}}^{\prime}-\widehat{\mathbf{R}}, \\
\delta R_{l k}=\widehat{R}_{l k}^{\prime}-\widehat{R}_{l k} .
\end{gathered}
$$

Substituting (32) in (26) and applying the first-order Taylor expansion of $P_{k l, \theta_{1}}(\Theta), P_{k l, \theta_{2}}(\Theta), P_{k l, \phi_{1}}(\Theta)$, and $P_{k l, \phi_{2}}(\Theta)$ yield

$$
\begin{aligned}
& \sum_{k=1}^{M} \sum_{l=1}^{M}\left(\left.P_{k l, \theta_{i}}(\Theta)\right|_{\Theta=\Theta^{(0)}}+\sum_{j=1}^{2}\left(\left.\delta \theta_{j} P_{k l, \theta_{i}, \theta_{j}}(\Theta)\right|_{\Theta=\Theta^{(0)}}\right)\right. \\
& \left.+\sum_{j=1}^{2}\left(\left.\delta \phi_{j} P_{k l, \theta_{i}, \theta_{j}}(\Theta)\right|_{\Theta=\Theta^{(0)}}\right) i=1,2\right)\left(\widehat{R}_{l k}\right. \\
& \left.+\delta R_{l k}\right)=0, \\
& \sum_{k=1}^{M} \sum_{l=1}^{M}\left(\left.P_{k l, \phi_{i}}(\Theta)\right|_{\Theta=\Theta^{(0)}}+\sum_{j=1}^{2}\left(\left.\delta \theta_{j} P_{k l, \phi_{i}, \theta_{j}}(\Theta)\right|_{\Theta=\Theta^{(0)}}\right)\right. \\
& \left.+\sum_{j=1}^{2}\left(\left.\delta \phi_{j} P_{k l, \phi_{i}, \theta_{j}}(\Theta)\right|_{\Theta=\Theta^{(0)}}\right) i=3,4\right)\left(\widehat{R}_{l k}\right. \\
& \left.\quad+\delta R_{l k}\right)=0 .
\end{aligned}
$$

Let $\mathbf{w}_{1}^{(u=1)}, \mathbf{w}_{2}^{(u=1)}, \mathbf{w}_{3}^{(u=1)}, \mathbf{w}_{4}^{(u=1)}, \mathbf{b}$, and $\mathbf{e}^{(u=1)}$ be defined as

$$
\begin{aligned}
& w_{i, j}^{(u=1)} \\
& = \begin{cases}\left.\sum_{k=1}^{M} \sum_{l=1}^{M} P_{k l, \theta_{j}, \theta_{i}}(\Theta)\right|_{\Theta=\Theta^{(0)}}\left(\widehat{R}_{l k}+\delta R_{l k}\right) & i=1,2 j=1,2 \\
\left.\sum_{k=1}^{M} \sum_{l=1}^{M} P_{k l, \phi_{j-2}, \theta_{i}}(\Theta)\right|_{\Theta=\Theta^{(0)}}\left(\widehat{R}_{l k}+\delta R_{l k}\right) & i=1,2 j=3,4 \\
\left.\sum_{k=1}^{M} \sum_{l=1}^{M} P_{k l, \theta_{j}, \phi_{i-2}}(\Theta)\right|_{\Theta=\Theta^{(0)}}\left(\widehat{R}_{l k}+\delta R_{l k}\right) & i=3,4 j=1,2 \\
\left.\sum_{k=1}^{M} \sum_{l=1}^{M} P_{k l, \phi_{j-2}, \phi_{i-2}}(\Theta)\right|_{\Theta=\Theta^{(0)}}\left(\widehat{R}_{l k}+\delta R_{l k}\right) & i=3,4 j=3,4,\end{cases} \\
& b_{i}= \begin{cases}-\left.\sum_{k=1}^{M} \sum_{l=1}^{M} P_{k l, \theta_{i}}(\Theta)\right|_{\Theta=\Theta^{(0)}}\left(\widehat{R}_{l k}+\delta R_{l k}\right) & i=1,2 \\
-\left.\sum_{k=1}^{M} \sum_{l=1}^{M} P_{k l, \phi_{i-2}}(\Theta)\right|_{\Theta=\Theta^{(0)}}\left(\widehat{R}_{l k}+\delta R_{l k}\right) & i=3,4,\end{cases} \\
& e_{i}^{(u=1)}= \begin{cases}\delta \theta_{i}^{(u=1)} & i=1,2 \\
\delta \phi_{i-2}^{(u=1)} & i=3,4,\end{cases}
\end{aligned}
$$

where $w_{i, j}^{(u=1)}$ denotes the $j$ th component of $\mathbf{w}_{i}$ and $b_{i}$ and $e_{i}$ denote the $i$ th component of $\mathbf{b}$ and $\mathbf{e}$, respectively. Using (34)(36), (33) can be expressed in matrix form

$$
\mathbf{W}^{(u=1)} \mathbf{e}^{(u=1)}=\mathbf{b},
$$

where $\mathbf{W}^{(u=1)}$ is defined as

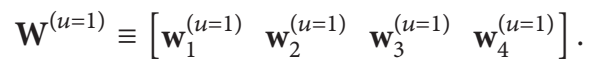

Note that $w_{i, j}^{(u=1)}$ in (34) denotes the entry at the $j$ th row and the $i$ th column of $\mathbf{W}$ in (38), not at the $i$ th row and the $j$ th column. $\operatorname{tr}(\mathbf{P}(\Theta) \widehat{\mathbf{R}})$ should be maximized at $\Theta=\Theta^{(0)}$, since, with the sample covariance matrix from noiseless response, the ML algorithm estimates the DOA accurately. Therefore, the partial derivatives of $\operatorname{tr}(\mathbf{P}(\Theta) \widehat{\mathbf{R}})$ with respect to the each entry of $\Theta$ should be zero at $\Theta=\widehat{\Theta}$

$$
\begin{aligned}
\frac{\partial}{\partial \theta_{i}} \operatorname{tr}\left(\left.\mathbf{P}(\Theta) \widehat{\mathbf{R}}\right|_{\Theta=\widehat{\Theta}}\right) & =\sum_{k=1}^{M} \sum_{l=1}^{M}\left(\left.\frac{\partial}{\partial \theta_{i}} P_{k l}(\Theta)\right|_{\Theta=\widehat{\Theta}}\right) \widehat{R}_{l k} \\
& =\sum_{k=1}^{M} \sum_{l=1}^{M}\left(\left.P_{k l, \theta_{i}}(\Theta)\right|_{\Theta=\widehat{\Theta}}\right) \widehat{R}_{l k} \\
& =0 \quad i=1,2, \\
\frac{\partial}{\partial \phi_{i}} \operatorname{tr}\left(\left.\mathbf{P}(\Theta) \widehat{\mathbf{R}}\right|_{\Theta=\widehat{\Theta}}\right) & =\sum_{k=1}^{M} \sum_{l=1}^{M}\left(\left.\frac{\partial}{\partial \phi_{i}} P_{k l}(\Theta)\right|_{\Theta=\widehat{\Theta}}\right) \widehat{R}_{l k} \\
& =\sum_{k=1}^{M} \sum_{l=1}^{M}\left(\left.P_{k l, \phi_{i}}(\Theta)\right|_{\Theta=\widehat{\Theta}}\right) \widehat{R}_{l k} \\
& =0 \quad i=1,2 .
\end{aligned}
$$

The last equalities of (39) can be expressed as

$$
\begin{aligned}
& \left.\sum_{k=1}^{M} \sum_{l=1}^{M} P_{k l, \theta_{i}}(\Theta)\right|_{\Theta=\Theta^{(0)}} \widehat{R}_{l k}=0 \quad i=1,2, \\
& \left.\sum_{k=1}^{M} \sum_{l=1}^{M} P_{k l, \phi_{i}}(\Theta)\right|_{\Theta=\Theta^{(0)}} \widehat{R}_{l k}=0 \quad i=1,2 .
\end{aligned}
$$

Substituting (40) in (35), b is simplified to

$$
b_{i}= \begin{cases}-\left.\sum_{k=1}^{M} \sum_{l=1}^{M} P_{k l, \theta_{i}}(\Theta)\right|_{\Theta=\Theta^{(0)}} \delta R_{l k} & i=1,2 \\ -\left.\sum_{k=1}^{M} \sum_{l=1}^{M} P_{k l, \phi_{i-2}}(\Theta)\right|_{\Theta=\Theta^{(0)}} \delta R_{l k} & i=3,4 .\end{cases}
$$

Using (38) and (35), $\mathbf{e}^{(u=1)}$ in (37) is defined as

$$
\mathbf{e}^{(u=1)}=\left(\mathbf{W}^{(u=1)}\right)^{-1} \mathbf{b} .
$$


Since $\widehat{R}_{l k} \gg \delta R_{l k}$ is true in (34), (34) can be approximated as

$$
\begin{aligned}
& w_{i, j}^{(u=1, v)} \\
& = \begin{cases}\left.\sum_{k=1}^{M} \sum_{l=1}^{M} P_{k l, \theta_{j}, \theta_{i}}(\Theta)\right|_{\Theta=\Theta^{(0)}} \widehat{R}_{l k} & i=1,2 j=1,2 \\
\left.\sum_{k=1}^{M} \sum_{l=1}^{M} P_{k l, \phi_{j-2}, \theta_{i}}(\Theta)\right|_{\Theta=\Theta^{(0)}} \widehat{R}_{l k} & i=1,2 j=3,4 \\
\left.\sum_{k=1}^{M} \sum_{l=1}^{M} P_{k l, \theta_{j}, \phi_{i-2}}(\Theta)\right|_{\Theta=\Theta^{(0)}} \widehat{R}_{l k} & i=3,4 j=1,2 \\
\left.\sum_{k=1}^{M} \sum_{l=1}^{M} P_{k l, \phi_{j-2}, \phi_{i-2}}(\Theta)\right|_{\Theta=\Theta^{(0)}} \widehat{R}_{l k} & i=3,4 j=3,4,\end{cases}
\end{aligned}
$$

where the superscript $v$ denotes that the approximation based on $\widehat{R}_{l k} \gg \delta R_{l k}$ is applied. Accordingly, $\mathbf{W}^{(u=1, v)}$ is defined as

$$
\mathbf{W}^{(u=1, v)}=\left[\begin{array}{llll}
\mathbf{w}_{1}^{(u=1, v)} & \mathbf{w}_{2}^{(u=1, v)} & \mathbf{w}_{3}^{(u=1, v)} & \mathbf{w}_{4}^{(u=1, v)}
\end{array}\right] .
$$

The first-order derivatives of $P_{k l, \theta_{1}}(\Theta), P_{k l, \theta_{2}}(\Theta), P_{k l, \phi_{1}}(\Theta)$, and $P_{k l, \phi_{2}}(\Theta)$ in (34) are derived in Appendix G. Applying $V$ approximation to (37), $\mathbf{e}^{(u=1, v)}$ is given by

$$
\mathbf{e}^{(u=1, v)}=\left(\mathbf{W}^{(u=1, v)}\right)^{-1} \mathbf{b},
$$

where an explicit expression of the entries of $\mathbf{e}^{(u=1, v)}$ is

$$
e_{i}^{(u=1, v)}= \begin{cases}\delta \theta_{i}^{(u=1, v)} & i=1,2 \\ \delta \phi_{i-2}^{(u=1, v)} & i=3,4 .\end{cases}
$$

\section{Closed-Form Expression of Mean Square Error}

Using (45), an analytic MSE of $\mathbf{e}^{(u=1, v)}$ can be expressed as

$$
\begin{aligned}
E & {\left[\mathbf{e}^{(u=1, v)}\left(\mathbf{e}^{(u=1, v)}\right)^{H}\right] } \\
& =\left(\mathbf{W}^{(u=1, v)}\right)^{-1} E\left[\mathbf{b b}^{H}\right]\left(\left(\mathbf{W}^{(u=1, v)}\right)^{-1}\right)^{H} .
\end{aligned}
$$

The entry at the $i$ th row and the $j$ th column of $E\left[\mathbf{b b} \mathbf{b}^{H}\right]$ can be expressed as

$$
\begin{aligned}
& \left(E\left[\mathbf{b}^{H} \mathbf{b}\right]\right)_{i, j} \\
& = \begin{cases}\sum_{k=1}^{M} \sum_{k^{\prime}=1}^{M} \sum_{l=1}^{M} \sum_{l^{\prime}=1}^{M} P_{k l, \theta_{i}, \theta_{j}} E\left[\delta R_{l k} \delta R_{l^{\prime} k^{\prime}}^{*}\right] & i=1,2 j=1,2 \\
\sum_{k=1}^{M} \sum_{k^{\prime}=1}^{M} \sum_{l=1}^{M} \sum_{l^{\prime}=1}^{M} P_{k l, \phi_{i-2,},} E\left[\delta R_{l k} \delta R_{l^{\prime} k^{\prime}}^{*}\right] & i=3,4 j=1,2 \\
\sum_{k=1}^{M} \sum_{k^{\prime}=1}^{M} \sum_{l=1}^{M} \sum_{l^{\prime}=1}^{M} P_{k l, \theta_{i}, \phi_{j-2}} E\left[\delta R_{l k} \delta R_{l^{\prime} k^{\prime}}^{*}\right] & i=1,2 j=3,4 \\
\sum_{k=1}^{M} \sum_{k^{\prime}=1}^{M} \sum_{l=1}^{M} \sum_{l^{\prime}=1}^{M} P_{k l, \phi_{i-2,}, \phi_{j-2}} E\left[\delta R_{l k} \delta R_{l^{\prime} k^{\prime}}^{*}\right] & i=3,4 j=3,4 .\end{cases}
\end{aligned}
$$

$E\left[\delta R_{l k} \delta R_{l^{\prime} k^{\prime}}^{*}\right]$ in (48) is derived in Appendix P. Entries of $E\left[\mathbf{e}^{(u=1, v)}\left(\mathbf{e}^{(u=1, v)}\right)^{H}\right]$ in (47) are written as

$$
\begin{aligned}
&\left(E\left[\mathbf{e}^{(u=1, v)}\left(\mathbf{e}^{(u=1, v)}\right)^{H}\right]\right)_{i, j} \\
& \quad= \begin{cases}E\left[\delta \theta_{i}^{(u=1, v)} \delta \theta_{j}^{*(u=1, v)}\right] & i=1,2 j=1,2 \\
E\left[\delta \theta_{i}^{(u=1, v)} \delta \phi_{j-2}^{*(u=1, v)}\right] & i=1,2 j=3,4 \\
E\left[\delta \phi_{i-2}^{(u=1, v)} \delta \theta_{j}^{*(u=1, v)}\right] & i=3,4 j=1,2 \\
E\left[\delta \phi_{i-2}^{(u=1, v)} \delta \phi_{j-2}^{*(u=1, v)}\right] & i=3,4 j=3,4 .\end{cases}
\end{aligned}
$$

From (49), it is clear that the MSEs of $\delta \theta_{1}, \delta \theta_{2}, \delta \phi_{1}$, and $\delta \phi_{2}$ are given by

$$
\begin{aligned}
& E\left[\left(\delta \theta_{i}^{(u=1, v)}\right)^{2}\right]=E\left[\delta \theta_{i}^{(u=1, v)}\left(\delta \theta_{i}^{(u=1, v)}\right)^{*}\right] \\
&=\left(E\left[\mathbf{e}^{(u=1, v)}\left(\mathbf{e}^{(u=1, v)}\right)^{H}\right]\right)_{i, i} \\
& E\left[\left(\delta \phi_{i-2}^{(u=1, v)}\right)^{2}\right]=E\left[\delta \phi_{i-2}^{(u=1, v)}\left(\delta \phi_{i-2}^{(u=1, v)}\right)^{*}\right] \\
&=\left(E\left[\mathbf{e}^{(u=1, v)}\left(\mathbf{e}^{(u=1, v)}\right)^{H}\right]\right)_{i, i} \\
& i=3,4,
\end{aligned}
$$

where $\left(\delta \theta_{i}^{(u=1, v)}\right)^{2}=\delta \theta_{i}^{(u=1, v)}\left(\delta \theta_{i}^{(u=1, v)}\right)^{*}$ and $\left(\delta \phi_{i}^{(u=1, v)}\right)^{2}=$ $\delta \phi_{i}^{(u=1, v)}\left(\delta \phi_{i}^{(u=1, v)}\right)^{*}$ are true since $\delta \theta_{i}$ and $\delta \phi_{i}$ are real-valued. Mathematical details on the derivation are summarized as follows.

Mathematical Details on the Performance Analysis of ML DOA Estimation. Number of snapshots is $L$, number of arrays is $M$, and number of signals is 2 .

$$
\begin{aligned}
& \Theta=\left(\theta_{1}, \phi_{1}, \theta_{2}, \phi_{2}\right), \\
& \Theta^{(0)}=\left(\theta_{1}^{(0)}, \phi_{1}^{(0)}, \theta_{2}^{(0)}, \phi_{2}^{(0)}\right), \\
& \widehat{\Theta}=\left(\widehat{\theta}_{1}, \widehat{\phi}_{1}, \widehat{\theta}_{2}, \widehat{\phi}_{2}\right) \Longrightarrow \\
& \delta \Theta \equiv \widehat{\Theta}-\Theta^{(0)}=\left(\delta \theta_{1}, \delta \phi_{1}, \delta \theta_{2}, \delta \phi_{2}\right), \\
& \mathbf{A}\left(\theta_{1}, \phi_{1}, \theta_{2}, \phi_{2}\right)=\left[\mathbf{a}\left(\theta_{1}, \phi_{1}\right) \mathbf{a}\left(\theta_{2}, \phi_{2}\right)\right], \\
& \mathbf{a}(\theta, \phi)=\left[a_{1}(\theta, \phi) \cdots a_{M}(\theta, \phi)\right]^{T} \\
& \quad=\left[\exp \left(j \psi_{1}(\theta, \phi)\right) \cdots \exp \left(j \psi_{M}(\theta, \phi)\right)\right]^{T}, \\
& \mathbf{s}(t)=\left[s_{1}(t), s_{2}(t)\right]^{T}, \\
& \mathbf{x}(t) \equiv \mathbf{A}(\Theta) \mathbf{s}(t), \\
& \mathbf{x}^{\prime}(t) \equiv \mathbf{A}(\Theta) \mathbf{s}(t)+\mathbf{n}(t) .
\end{aligned}
$$


Projection matrix of $\mathbf{A}(\Theta) \quad$ : $\mathbf{P}(\Theta)=$ $\mathbf{A}(\Theta)\left(\mathbf{A}^{H}(\Theta) \mathbf{A}(\Theta)\right)^{-1} \mathbf{A}^{H}(\Theta)$

$$
\begin{aligned}
\widehat{\mathbf{R}} & =\frac{1}{L} \sum_{i=1}^{L} \mathbf{x}\left(t_{i}\right) \mathbf{x}^{H}\left(t_{i}\right), \\
\widehat{\mathbf{R}}^{\prime} & =\frac{1}{L} \sum_{i=1}^{L} \mathbf{x}^{\prime}\left(t_{i}\right) \mathbf{x}^{\prime H}\left(t_{i}\right) \Longrightarrow \\
\delta \mathbf{R} & \equiv \widehat{\mathbf{R}}^{\prime}-\widehat{\mathbf{R}} .
\end{aligned}
$$

The derivatives of the ML cost function, based on the covariance matrix associated with noisy array response, with respect to each incident signal, are not identically zero at true incident angles. From the angles at which the derivative is identically zero, the expression of the estimation error can be derived

$$
\begin{aligned}
P_{k l, \theta_{n}}(\Theta) & \equiv \frac{\partial}{\partial \theta_{n}} \mathbf{P}_{k l}(\Theta), \\
P_{k l, \phi_{n}}(\Theta) & \equiv \frac{\partial}{\partial \phi_{n}} \mathbf{P}_{k l}(\Theta), \\
\operatorname{tr}\left(\mathbf{P}_{k l}(\Theta) \widehat{\mathbf{R}}^{\prime}\right) & =\sum_{k=1}^{M} \sum_{l=1}^{M} P_{k l}(\Theta) \widehat{R}_{l k}^{\prime} .
\end{aligned}
$$

The derivative of the ML cost function, based on the covariance matrix associated with noiseless array response, with respect to each incident signal, is identically zero at true incident angles

$$
\begin{aligned}
& \left.\frac{\partial}{\partial \theta_{n}} \operatorname{tr}\left(\mathbf{P}_{k l}(\Theta) \widehat{\mathbf{R}}\right)\right|_{\Theta=\Theta^{(0)}}=\left.\sum_{k=1}^{M} \sum_{l=1}^{M} P_{k l, \theta_{n}}(\Theta) \widehat{R}_{l k}\right|_{\Theta=\Theta^{(0)}} \\
& \quad=0, \quad n=1,2, \\
& \left.\frac{\partial}{\partial \phi_{n}} \operatorname{tr}\left(\mathbf{P}_{k l}(\Theta) \widehat{\mathbf{R}}\right)\right|_{\Theta=\Theta^{(0)}}=\left.\sum_{k=1}^{M} \sum_{l=1}^{M} P_{k l, \phi_{n}}(\Theta) \widehat{R}_{l k}\right|_{\Theta=\Theta^{(0)}} \\
& =0, \quad n=1,2,\left.\quad\right|^{\frac{\partial}{\partial \theta_{n}}} \operatorname{tr}\left(\left.\mathbf{P}_{k l}(\Theta)\right|_{\Theta=\widehat{\Theta}} \widehat{\mathbf{R}}^{\prime}\right)=\operatorname{tr}\left(\left.\frac{\partial}{\partial \theta_{n}} \mathbf{P}_{k l}(\Theta)\right|_{\Theta=\widehat{\mathbb{R}}^{\prime}} \widehat{\mathbf{R}}^{\prime}\right) \\
& =\left.\sum_{k=1}^{M} \sum_{l=1}^{M} P_{k l, \theta_{n}}(\Theta)\left(\widehat{R}_{l k}+\delta R_{l k}\right)\right|_{\Theta=\widehat{\Theta}}=0,
\end{aligned}
$$

$$
\begin{aligned}
& \left.\sum_{k=1}^{M} \sum_{l=1}^{M}\left(g_{n}\right)_{k l}(\Theta)\left(\widehat{R}_{l k}+\delta R_{l k}\right)\right|_{\Theta=\Theta^{(0)}+\delta \Theta} \\
& \quad=\sum_{k=1}^{M} \sum_{l=1}^{M}\left(\left.\left(g_{n}\right)_{k l}(\Theta)\right|_{\Theta=\Theta^{(0)}}\right. \\
& \quad+\left.\delta \theta_{1} \frac{\partial}{\partial \theta_{1}}\left(g_{n}\right)_{k l}(\Theta)\right|_{\Theta=\Theta^{(0)}} \\
& \quad+\left.\delta \theta_{2} \frac{\partial}{\partial \theta_{2}}\left(g_{n}\right)_{k l}(\Theta)\right|_{\Theta=\Theta^{(0)}} \\
& \quad+\left.\delta \phi_{1} \frac{\partial}{\partial \phi_{1}}\left(g_{n}\right)_{k l}(\Theta)\right|_{\Theta=\Theta^{(0)}} \\
& \quad+\left.\delta \phi_{2} \frac{\partial}{\partial \phi_{2}}\left(g_{n}\right)_{k l}(\Theta)\right|_{\Theta=\Theta^{(0)}}\left(\widehat{R}_{l k}+\delta R_{l k}\right)=0
\end{aligned}
$$$$
n=1, \ldots, N
$$

Substituting (55) and (56) in (58) results in

$$
\left[\begin{array}{lllll}
\left.\frac{\partial}{\partial \theta_{1}}\left(f_{1}\right)_{k l}(\Theta)\right|_{\Theta=\Theta^{(0)}}\left(\widehat{R}_{l k}+\delta R_{l k}\right) & \left.\frac{\partial}{\partial \theta_{2}}\left(f_{1}\right)_{k l}(\Theta)\right|_{\Theta=\Theta^{(0)}}\left(\widehat{R}_{l k}+\delta R_{l k}\right) & \left.\frac{\partial}{\partial \phi_{1}}\left(f_{1}\right)_{k l}(\Theta)\right|_{\Theta=\Theta^{(0)}}\left(\widehat{R}_{l k}+\delta R_{l k}\right) & \left.\frac{\partial}{\partial \phi_{2}}\left(f_{1}\right)_{k l}(\Theta)\right|_{\Theta=\Theta^{(0)}}\left(\widehat{R}_{l k}+\delta R_{l k}\right) \\
\left.\frac{\partial}{\partial \theta_{1}}\left(f_{2}\right)_{k l}(\Theta)\right|_{\Theta=\Theta^{(0)}}\left(\widehat{R}_{l k}+\delta R_{l k}\right) & \left.\frac{\partial}{\partial \theta_{2}}\left(f_{2}\right)_{k l}(\Theta)\right|_{\Theta=\Theta^{(0)}}\left(\widehat{R}_{l k}+\delta R_{l k}\right) & \left.\frac{\partial}{\partial \phi_{1}}\left(f_{2}\right)_{k l}(\Theta)\right|_{\Theta=\Theta^{(0)}}\left(\widehat{R}_{l k}+\delta R_{l k}\right) & \left.\frac{\partial}{\partial \phi_{2}}\left(f_{2}\right)_{k l}(\Theta)\right|_{\Theta=\Theta^{(0)}}\left(\widehat{R}_{l k}+\delta R_{l k}\right) \\
\left.\frac{\partial}{\partial \theta_{1}}\left(g_{1}\right)_{k l}(\Theta)\right|_{\Theta=\Theta^{(0)}}\left(\widehat{R}_{l k}+\delta R_{l k}\right) & \left.\frac{\partial}{\partial \theta_{2}}\left(g_{1}\right)_{k l}(\Theta)\right|_{\Theta=\Theta^{(0)}}\left(\widehat{R}_{l k}+\delta R_{l k}\right) & \left.\frac{\partial}{\partial \phi_{1}}\left(f_{2}\right)_{k l}(\Theta)\right|_{\Theta=\Theta^{(0)}}\left(\widehat{R}_{l k}+\delta R_{l k}\right) & \left.\frac{\partial}{\partial \phi_{2}}\left(f_{2}\right)_{k l}(\Theta)\right|_{\Theta=\Theta^{(0)}}\left(\widehat{R}_{l k}+\delta R_{l k}\right) \\
\left.\frac{\partial}{\partial \theta_{1}}\left(g_{2}\right)_{k l}(\Theta)\right|_{\Theta=\Theta^{(0)}}\left(\widehat{R}_{l k}+\delta R_{l k}\right) & \left.\frac{\partial}{\partial \theta_{2}}\left(g_{2}\right)_{k l}(\Theta)\right|_{\Theta=\Theta^{(0)}}\left(\widehat{R}_{l k}+\delta R_{l k}\right) & \left.\frac{\partial}{\partial \phi_{1}}\left(g_{2}\right)_{k l}(\Theta)\right|_{\Theta=\Theta^{(0)}}\left(\widehat{R}_{l k}+\delta R_{l k}\right) & \left.\frac{\partial}{\partial \phi_{2}}\left(g_{2}\right)_{k l}(\Theta)\right|_{\Theta=\Theta^{(0)}}\left(\widehat{R}_{l k}+\delta R_{l k}\right)
\end{array}\right]\left[\begin{array}{l}
\delta \theta_{1}^{(v=1)} \\
\delta \phi_{1}^{(v=1)} \\
\delta \phi_{N}^{(v=1)}
\end{array}\right]
$$




$$
=\left[\begin{array}{c}
-\left.\sum_{k=1}^{M} \sum_{l=1}^{M}\left(f_{1}\right)_{k l}(\Theta)\right|_{\Theta=\Theta^{(0)}} \delta R_{l k} \\
-\left.\sum_{k=1}^{M} \sum_{l=1}^{M}\left(f_{2}\right)_{k l}(\Theta)\right|_{\Theta=\Theta^{(0)}} \delta R_{l k} \\
-\left.\sum_{k=1}^{M} \sum_{l=1}^{M}\left(g_{1}\right)_{k l}(\Theta)\right|_{\Theta=\Theta^{(0)}} \delta R_{l k} \\
-\left.\sum_{k=1}^{M} \sum_{l=1}^{M}\left(g_{2}\right)_{k l}(\Theta)\right|_{\Theta=\Theta^{(0)}} \delta R_{l k}
\end{array}\right] .
$$

Using $\widehat{R}_{l k}+\delta R_{l k} \approx \widehat{R}_{l k}$ in (59) results in

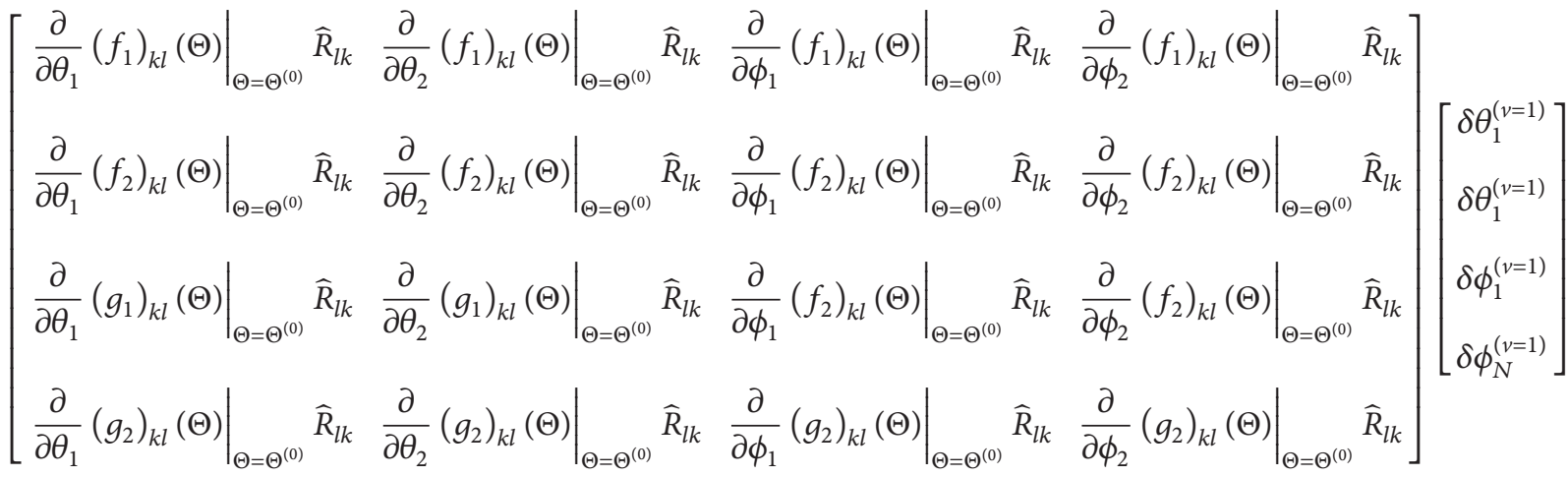

$$
\begin{aligned}
& {\left[\begin{array}{c}
-\left.\sum_{k=1}^{M} \sum_{l=1}^{M}\left(f_{1}\right)_{k l}(\Theta)\right|_{\Theta=\Theta^{(0)}} \delta R_{l k} \\
-\left.\sum_{k=1}^{M} \sum_{l=1}^{M}\left(f_{2}\right)_{k l}(\Theta)\right|_{\Theta=\Theta^{(0)}} \delta R_{l k} \\
-\left.\sum_{k=1}^{M} \sum_{l=1}^{M}\left(g_{1}\right)_{k l}(\Theta)\right|_{\Theta=\Theta^{(0)}} \delta R_{l k} \\
-\left.\sum_{k=1}^{M} \sum_{l=1}^{M}\left(g_{2}\right)_{k l}(\Theta)\right|_{\Theta=\Theta^{(0)}} \delta R_{l k}
\end{array}\right] \Longrightarrow} \\
& \mathbf{W}^{(v=1)} \mathbf{e}^{(v=1)}=\mathbf{b}, \\
& \mathbf{e}^{(v=1)}=\left(\mathbf{W}^{(v=1)}\right)^{-1} \mathbf{b}, \\
& E\left[\mathbf{e}^{(v=1)}\left(\mathbf{e}^{(v=1)}\right)^{H}\right]=E\left[\left(\mathbf{W}^{(v=1)}\right)^{-1} \mathbf{b}\left(\left(\mathbf{W}^{(v=1)}\right)^{-1} \mathbf{b}\right)^{H}\right]=E\left[\left(\mathbf{W}^{(v=1)}\right)^{-1} \mathbf{b b}^{H}\left(\left(\mathbf{W}^{(v=1)}\right)^{-1}\right)^{H}\right] \\
& =\left(\mathbf{W}^{(v=1)}\right)^{-1} E\left[\mathbf{b b}^{H}\right]\left(\left(\mathbf{W}^{(v=1)}\right)^{-1}\right)^{H} .
\end{aligned}
$$


An analytic expression of $E\left[\mathbf{b} \mathbf{b}^{H}\right]$ can be derived by exploiting statistical distribution of $\delta R_{l k}$.

\section{Numerical Results}

MSEs of $\widehat{\theta}_{1}, \widehat{\theta}_{1}^{(u=1)}$, and $\widehat{\theta}_{1}^{(u=1, v)}$ are defined as

$$
\begin{gathered}
\text { Simulation } E\left[\left(\widehat{\theta}_{1}-\theta_{1}^{(0)}\right)^{2}\right]=\frac{1}{T} \sum_{t=1}^{T}\left(\delta \theta_{1,(t)}\right)^{2}, \\
\text { Simulation } E\left[\left(\hat{\theta}_{1}^{(u=1)}-\theta_{1}^{(0)}\right)^{2}\right]=\frac{1}{T} \sum_{t=1}^{T}\left(\delta \theta_{1,(t)}^{(u=1)}\right)^{2}, \\
\text { Simulation } E\left[\left(\hat{\theta}_{1}^{(u=1, v)}-\theta_{1}^{(0)}\right)^{2}\right]=\frac{1}{T} \sum_{t=1}^{T}\left(\delta \theta_{1,(t)}^{(u=1, v)}\right)^{2},
\end{gathered}
$$

where $T$ denotes the number of repetitions. The subscript $t$ denotes the estimate associated with the $t$ th repetition out of $T$ repetitions. $\left(\delta \theta_{1,(t)}\right)^{2},\left(\delta \theta_{1,(t)}^{(u=1)}\right)^{2}$, and $\left(\delta \theta_{1,(t)}^{(u=1, v)}\right)^{2}$ in (61) are given by (42), (45), and (13), respectively.

In Figure 2(a), we illustrate how accurate the MSEs of $\widehat{\theta}_{1}, \widehat{\theta}_{1}^{(u=1)}$, and $\widehat{\theta}_{1}^{(u=1, v)}$ are. The results with "Simulation $E\left[\left(\widehat{\theta}_{1}-\theta_{1}^{(0)}\right)^{2}\right]$," "Simulation $E\left[\left(\widehat{\theta}_{1}^{(u=1)}-\theta_{1}^{(0)}\right)^{2}\right]$," "Simulation $E\left[\left(\widehat{\theta}_{1}^{(u=1, v)}-\theta_{1}^{(0)}\right)^{2}\right]$, " and "Analytic $E\left[\left(\hat{\theta}_{1}^{(u=1, v)}-\theta_{1}^{(0)}\right)^{2}\right]$ " are obtained using (61) and (50), respectively. It has been clearly shown that the results with "Simulation $E\left[\left(\widehat{\theta}_{1}^{(u=1, v)}-\theta_{1}^{(0)}\right)^{2}\right]$ " show good agreement with those with "Analytic $E\left[\left(\widehat{\theta}_{1}^{(u=1, v)}-\right.\right.$ $\left.\left.\theta_{1}^{(0)}\right)^{2}\right]$ ". In addition, the results with "Simulation $E\left[\left(\widehat{\theta}_{1}^{(u=1, v)}-\right.\right.$ $\left.\left.\theta_{1}^{(0)}\right)^{2}\right]$ " get closer to those with "Simulation $E\left[\left(\hat{\theta}_{1}-\theta_{1}^{(0)}\right)^{2}\right]$ " as SNR increases. Therefore, at high SNR, the results with "Analytic $E\left[\left(\widehat{\theta}_{1}^{(u=1, v)}-\theta_{1}^{(0)}\right)^{2}\right]$ " can be used for determining how accurate the results with " $E\left[\left(\hat{\theta}_{1}-\theta_{1}^{(0)}\right)^{2}\right]$ " are. The lower bound of the MSE for an unbiased estimator is called the CRLB. The CRLB for $\theta_{1}$ is also illustrated in Figure 2(a), where it is verified that the CRLB is actually below the analytic MSEs. Generally, the CRLB of each parameter can be obtained from the diagonal entry of $\mathbf{B}_{\mathrm{STO}}$ :

$$
\begin{aligned}
& E\left[\left\{\widehat{\Theta}-\Theta^{(0)}\right\}\left\{\widehat{\Theta}-\Theta^{(0)}\right\}^{T}\right] \geq \mathbf{B}_{\text {STO }} \\
& \left(\mathbf{B}_{\mathrm{STO}}^{-1}\right)_{i j}=\frac{2 N}{\sigma^{2}} \operatorname{Re}\left[\operatorname{tr}\left\{\mathbf{A}_{j}^{H} \mathbf{P}_{\mathbf{A}}^{\perp} \mathbf{A}_{i} \mathbf{S} \mathbf{A}^{H} \mathbf{R}^{-1} \mathbf{A S}\right\}\right], \\
& \quad i, j=1, \ldots, 4,
\end{aligned}
$$

where $\mathbf{A}_{i}$ is defined in [13]. The CRLB of $\theta_{1}$ is obtained from $(1,1)$ element of $\mathbf{B}_{\text {STO }}$ in $(63)$.

$$
\begin{gathered}
\text { MSEs of } \widehat{\theta}_{2}, \widehat{\theta}_{2}^{(u=1)} \text {, and } \widehat{\theta}_{2}^{(u=1, v)} \text { are defined as } \\
\text { Simulation } E\left[\left(\widehat{\theta}_{2}-\theta_{2}^{(0)}\right)^{2}\right]=\frac{1}{T} \sum_{t=1}^{T}\left(\delta \theta_{2,(t)}^{2}\right)^{2}, \\
\text { Simulation } E\left[\left(\widehat{\theta}_{2}^{(u=1)}-\theta_{2}^{(0)}\right)^{2}\right]=\frac{1}{T} \sum_{t=1}^{T}\left(\delta \theta_{2,(t)}^{(u=1)}\right)^{2}, \\
\text { Simulation } E\left[\left(\hat{\theta}_{2}^{(u=1, v)}-\theta_{2}^{(0)}\right)^{2}\right]=\frac{1}{T} \sum_{t=1}^{T}\left(\delta \theta_{2,(t)}^{(u=1, v)}\right)^{2} .
\end{gathered}
$$

$\left(\delta \theta_{2,(t)}\right)^{2},\left(\delta \theta_{2,(t)}^{(u=1)}\right)^{2}$, and $\left(\delta \theta_{2,(t)}^{(u=1, v)}\right)^{2}$ in (64) are given by (13), (42), and (45), respectively. In Figure 2(b), we illustrate how accurate the MSEs of $\widehat{\theta}_{2}, \widehat{\theta}_{2}^{(u=1)}$, and $\widehat{\theta}_{2}^{(u=1, v)}$ are. The results with "Simulation $E\left[\left(\widehat{\theta}_{2}-\theta_{2}^{(0)}\right)^{2}\right]$," "Simulation $E\left[\left(\hat{\theta}_{2}^{(u=1)}-\theta_{2}^{(0)}\right)^{2}\right]$," “Simulation $E\left[\left(\hat{\theta}_{2}^{(u=1, v)}-\theta_{2}^{(0)}\right)^{2}\right]$," and "Analytic $E\left[\left(\widehat{\theta}_{2}^{(u=1, v)}-\theta_{2}^{(0)}\right)^{2}\right]$ " are obtained using (64) and (51), respectively. We can easily see that the observation for $\widehat{\theta}_{1} \widehat{\theta}_{1}^{(u=1)}$ and $\widehat{\theta}_{1}^{(u=1, v)}$ in Figure 2(a) is also true for $\widehat{\theta}_{2} \widehat{\theta}_{2}^{(u=1)}$ and $\widehat{\theta}_{2}^{(u=1, v)}$ in Figure 2(b). The CRLB of $\theta_{2}$ is obtained from the $(3,3)$ element of the $\mathbf{B}_{\text {STO }}$ in $(63)$.

MSEs of the $\widehat{\phi}_{1}, \widehat{\phi}_{1}^{(u=1)}$, and $\widehat{\phi}_{1}^{(u=1, v)}$ are defined as

Simulation $E\left[\left(\widehat{\phi}_{1}-\phi_{1}^{(0)}\right)^{2}\right]=\frac{1}{T} \sum_{t=1}^{T}\left(\delta \phi_{1,(t)}^{2}\right)^{2}$,

Simulation $E\left[\left(\hat{\phi}_{1}^{(u=1)}-\phi_{1}^{(0)}\right)^{2}\right]=\frac{1}{T} \sum_{t=1}^{T}\left(\delta \phi_{1,(t)}^{(u=1)}\right)^{2}$,

Simulation $E\left[\left(\hat{\phi}_{1}^{(u=1, v)}-\phi_{1}^{(0)}\right)^{2}\right]$

$$
=\frac{1}{T} \sum_{t=1}^{T}\left(\delta \phi_{1,(t)}^{(u=1, v)}\right)^{2} .
$$

$\left(\delta \phi_{1,(t)}\right)^{2},\left(\delta \phi_{1,(t)}^{(u=1)}\right)^{2}$, and $\left(\delta \phi_{1,(t)}^{(u=1, v)}\right)^{2}$ in (65) are given by (13), (42), and (45), respectively.

In Figure 2(c), we illustrate how accurate the MSEs of $\widehat{\phi}_{1}, \widehat{\phi}_{1}^{(u=1)}$, and $\hat{\phi}_{1}^{(u=1, v)}$ are. The results with "Simulation $E\left[\left(\widehat{\phi}_{1}-\phi_{1}^{(0)}\right)^{2}\right]$," "Simulation $E\left[\left(\widehat{\phi}_{1}^{(u=1)}-\phi_{1}^{(0)}\right)^{2}\right]$," "Simulation $E\left[\left(\widehat{\phi}_{1}^{(u=1, v)}-\phi_{1}^{(0)}\right)^{2}\right]$," and "Analytic $E\left[\left(\widehat{\phi}_{1}^{(u=1, v)}-\phi_{1}^{(0)}\right)^{2}\right]$ " are obtained using (65) and (51), respectively. It is clear in Figure $2(\mathrm{c})$ that $\widehat{\phi}_{1}^{(u=1, v)}$ can be used to approximate $\widehat{\phi}_{1}$ and that empirical MSE of $\widehat{\phi}_{1}^{(u=1, v)}$ is equal to an analytic MSE of $\widehat{\phi}_{1}^{(u=1, v)}$ in (51). The CRLB of $\phi_{1}$ is obtained from the $(2,2)$ element of $\mathbf{B}_{\mathrm{STO}}$ in (63).

MSEs of $\widehat{\phi}_{2}, \widehat{\phi}_{2}^{(u=1)}$, and $\widehat{\phi}_{2}^{(u=1, v)}$ are defined as

Simulation $E\left[\left(\hat{\phi}_{2}-\phi_{2}^{(0)}\right)^{2}\right]=\frac{1}{T} \sum_{t=1}^{T}\left(\delta \phi_{2,(t)}\right)^{2}$,

Simulation $E\left[\left(\hat{\phi}_{2}^{(u=1)}-\phi_{2}^{(0)}\right)^{2}\right]=\frac{1}{T} \sum_{t=1}^{T}\left(\delta \phi_{2,(t)}^{(u=1)}\right)^{2}$,

Simulation $E\left[\left(\widehat{\phi}_{2}^{(u=1, v)}-\phi_{2}^{(0)}\right)^{2}\right]$

$$
=\frac{1}{T} \sum_{t=1}^{T}\left(\delta \phi_{2,(t)}^{(u=1, v)}\right)^{2} .
$$

$\left(\delta \phi_{2,(t)}\right)^{2},\left(\delta \phi_{2,(t)}^{(u=1)}\right)^{2}$, and $\left(\delta \phi_{2,(t)}^{(u=1, v)}\right)^{2}$ in (66) are given by (13), (42), and (45), respectively. In Figure 2(d), we illustrate how accurate the MSEs of $\widehat{\phi}_{2}, \widehat{\phi}_{2}^{(u=1)}$, and $\widehat{\phi}_{2}^{(u=1, v)}$ are. 

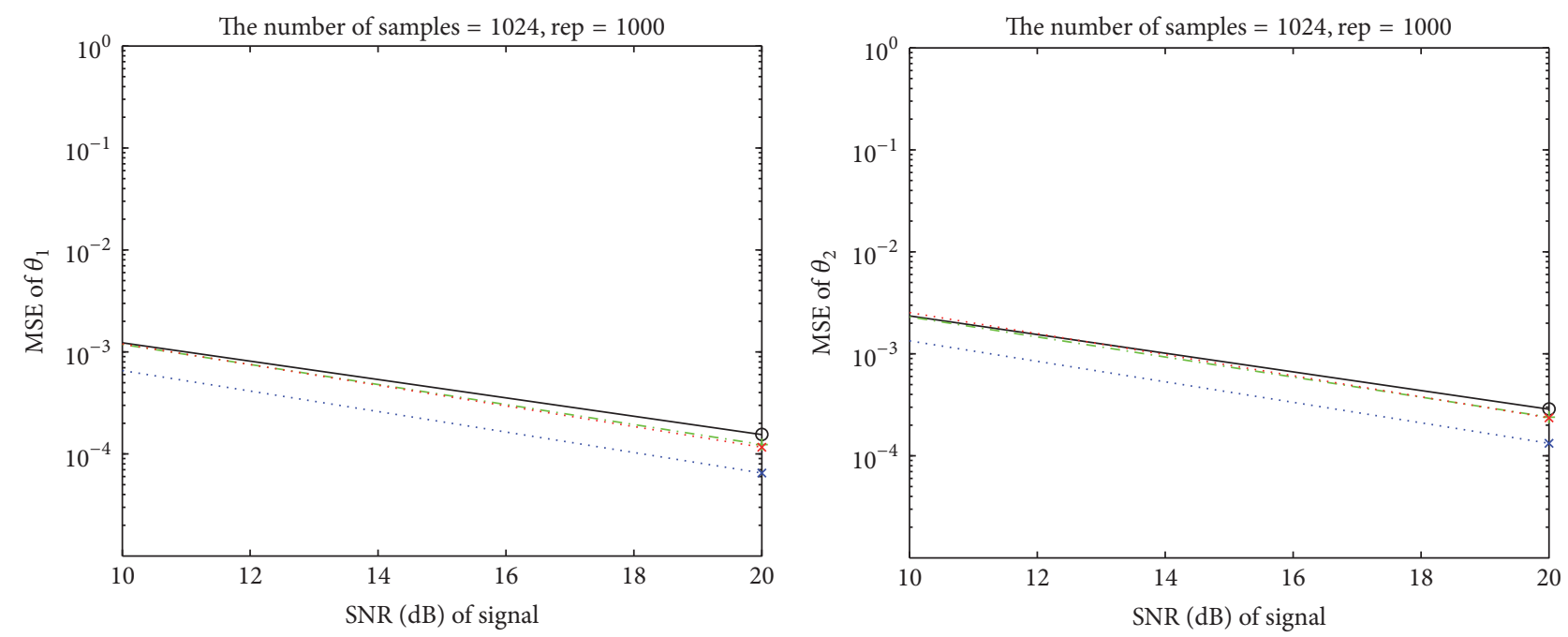

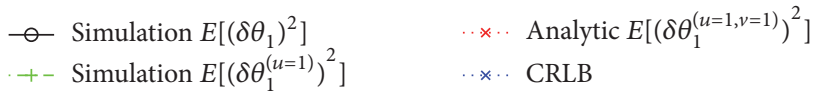

(a) Mean square error of $\theta_{1}$

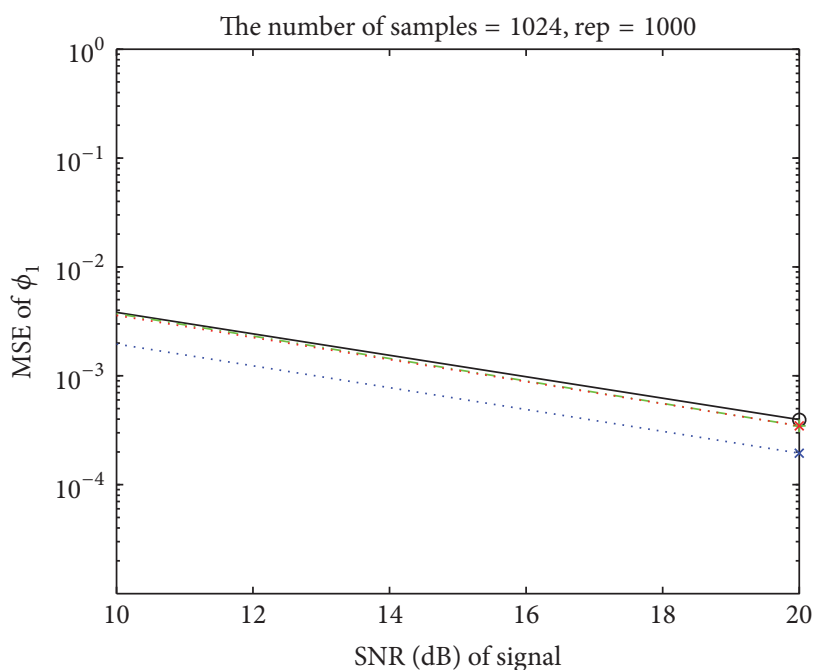

$\begin{array}{ll}\curvearrowleft \text { Simulation } E\left[\left(\delta \phi_{1}\right)^{2}\right] & \ldots * \cdot \text { Analytic } E\left[\left(\delta \phi_{1}^{(u=1, v=1)}\right)^{2}\right] \\ +- \text { Simulation } E\left[\left(\delta \phi_{1}^{(u=1)}\right)^{2}\right] & \cdots * \cdot \text { CRLB }\end{array}$

(c) Mean square error of $\phi_{1}$

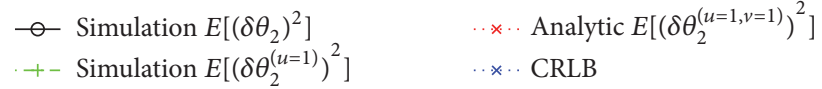

(b) Mean square error of $\theta_{2}$

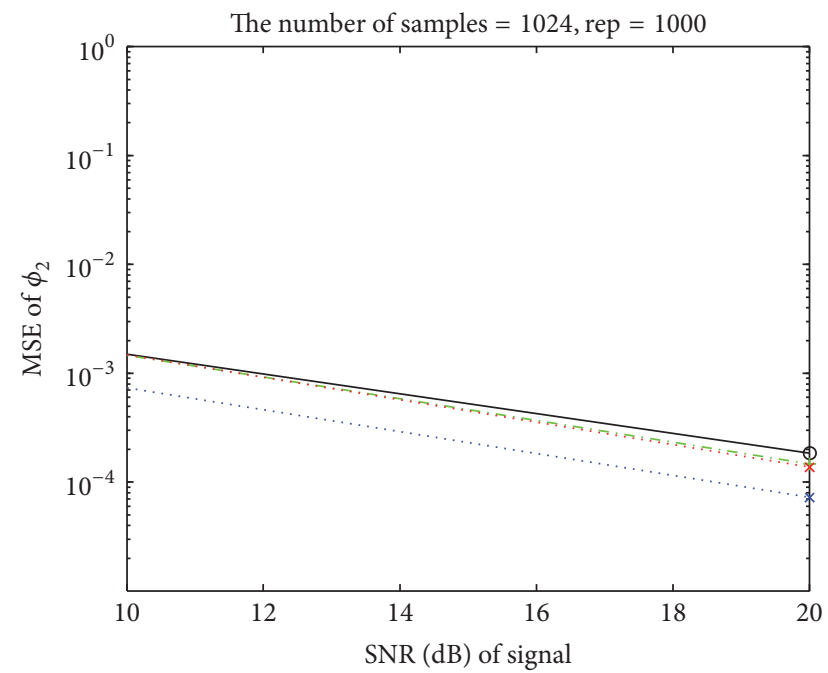

$\begin{array}{lll}- \text { Simulation } E\left[\left(\delta \phi_{2}\right)^{2}\right] & \cdots * \cdots & \text { Analytic } E\left[\left(\delta \phi_{2}^{(u=1, v=1)}\right)^{2}\right] \\ +- \text { Simulation } E\left[\left(\delta \phi_{2}^{(u=1)}\right)^{2}\right] & \cdots * \cdots & \text { CRLB }\end{array}$

(d) Mean square error of $\phi_{2}$

Figure 2: Mean square error of $\theta_{1}, \theta_{2}, \phi_{1}$, and $\phi_{2}$ for SNR $10 \mathrm{~dB}$ to $20 \mathrm{~dB}$.

The results with "Simulation $E\left[\left(\widehat{\phi}_{2}-\phi_{2}^{(0)}\right)^{2}\right]$," "Simulation $E\left[\left(\widehat{\phi}_{2}^{(u=1)}-\phi_{2}^{(0)}\right)^{2}\right]$," "Simulation $E\left[\left(\widehat{\phi}_{2}^{(u=1, v)}-\phi_{2}^{(0)}\right)^{2}\right]$," and "Analytic $E\left[\left(\widehat{\phi}_{2}^{(u=1, v)}-\phi_{2}^{(0)}\right)^{2}\right]$ " are obtained using (66) and (51), respectively. It is clear that the observation on the various estimates of $\phi_{1}$, in Figure 2(c), also applies to the various estimates of $\phi_{2}$ in Figure 2(d). The CRLB of $\phi_{2}$ is obtained from the $(4,4)$ element of $\mathbf{B}_{\mathrm{STO}}$ in $(63)$.

In Figures 3-5, the results with respect to SNR are shown. It has been shown in the new numerical results that a gap between the MSE of the MLE and the Cramer-Rao lower bound gets smaller as the SNR varies from $-20 \mathrm{~dB}$ to $20 \mathrm{~dB}$ in increments of $10 \mathrm{~dB}$. Note that, in Figure 3, the gap for $\mathrm{SNR}=20 \mathrm{~dB}$ is much smaller than that for $\mathrm{SNR}=-20 \mathrm{~dB}$, which implies that the MSE of the MLE attains CRLB as SNR increases. Similar observation can be found in Figures 4 and 5.

Closed-form expression is only available for the estimate both with $U$-approximation and $V$-approximation. $U$ approximation in our scheme is based on the first-order Taylor series expansion, which implies that $U$-approximation becomes less accurate when the variance of the noise is large since the first-order Taylor series expansion does not take into account higher-order terms. Note that high variance of noise 

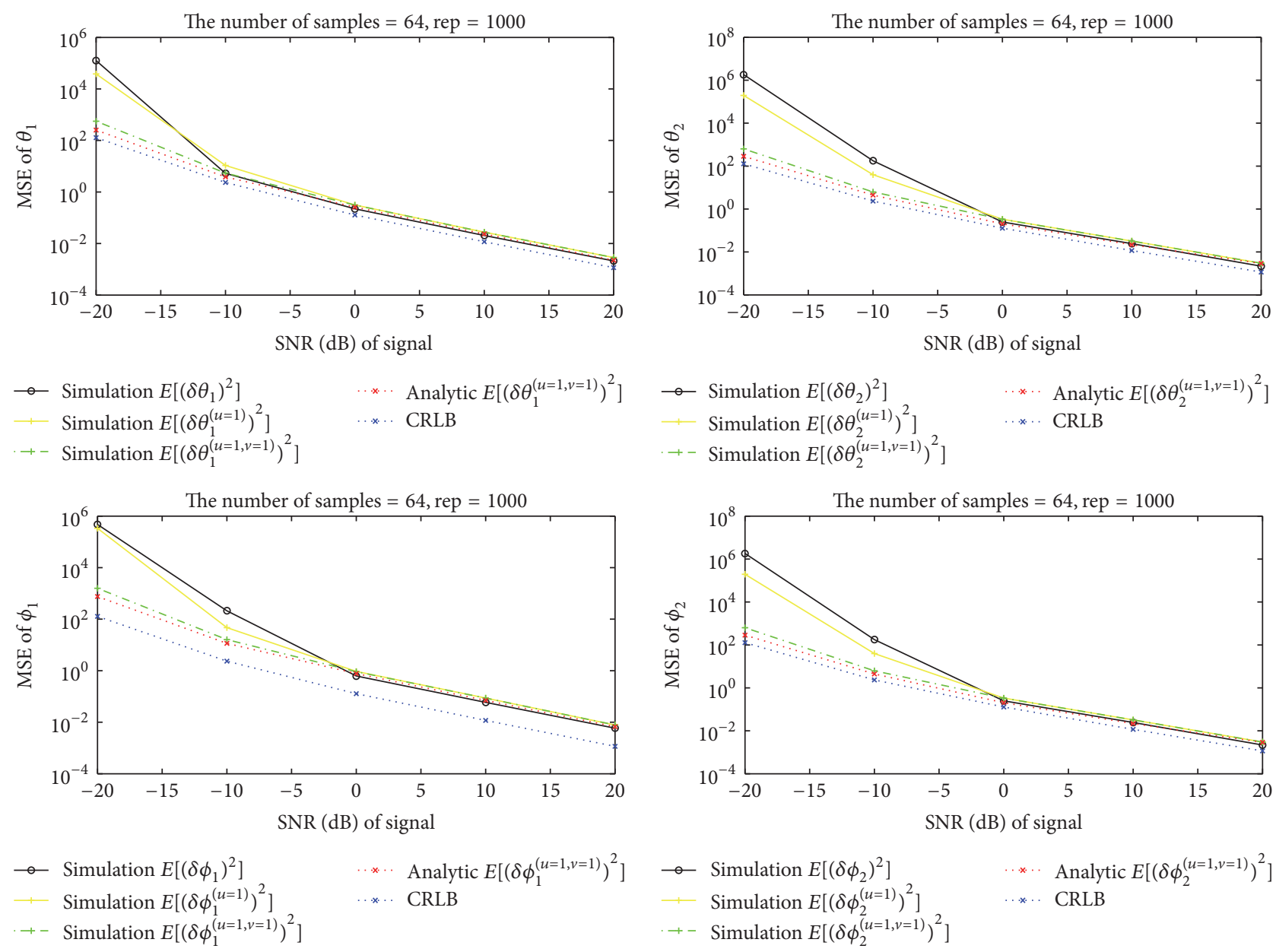

Figure 3: MSE of $\theta_{1}, \theta_{2}, \phi_{1}$, and $\phi_{2}$ for SNR $-20 \mathrm{~dB}$ to $20 \mathrm{~dB}$ (the number of samples $=2^{6}$ ).

corresponds to low SNR. $V$-approximation in our scheme is based on the fact that the difference between the covariance matrix with noisy array response and that with noiseless antenna response is much smaller than the covariance matrix with noiseless antenna response. $V$-approximation is accurate when the noisy antenna response is similar to the noiseless antenna response, which is true for high SNR. That is, $V$ approximation is valid for high SNR.

In conclusion, both $U$-approximation and $V$-approximation are valid for high SNR, and therefore the closed-form expression for the estimate with $U$-approximation and $V$ approximation is also valid for high SNR.

\section{Summary and Conclusion}

In Figures 6 and 7, we illustrate the outline of our derivation and how the performance analysis of the ML algorithm method is conducted.

In the case of azimuth/elevation estimation of multiple incident signals using the UCA, it has been shown that the bias of the estimate associated with the ML algorithm can be expressed in the closed-form at high SNR where the estimation error is small. The closed-form expression of the MSE of the one of the estimates has been derived, which implies that the MSE of the estimates can be obtained without computationally intensive Monte-Carlo simulation. The derivation is based on the fact that, for small estimation error, we can make the Taylor series approximation of the array vector, which makes it possible for us to get a closed-form expression of the MSE of the estimate.

We assume that an additive noise on the antenna elements is complex Gaussian distributed and that noise at each antenna is spatially white. Future works include how to extend the results in this paper to the case of non-Gaussian noise and spatially correlated noise. Although the formulation and the numerical results for two incident signals are presented in this paper, an extension to multiple incident signals is quite intuitively clear and straightforward. The number of columns $\mathbf{A}(\Theta)$ in (4) is equal to the number of incident signals. For $N$ incident signals, $\mathbf{A}^{\mathbf{H}}(\boldsymbol{\Theta}) \mathbf{A}(\Theta)$ in (14) is $N \times N$ matrix and $\left(\mathbf{A}^{\mathbf{H}}(\boldsymbol{\Theta}) \mathbf{A}(\boldsymbol{\Theta})\right)^{-1}$ in (17) can be explicitly expressed using the adjoint method. More specifically, $D(\Theta)$ in (18), which is a determinant of $\mathbf{A}^{\mathbf{H}}(\boldsymbol{\Theta}) \mathbf{A}(\boldsymbol{\Theta})$, can be obtained from the cofactor expansion along any row or any column of $\mathbf{A}^{\mathbf{H}}(\boldsymbol{\Theta}) \mathbf{A}(\boldsymbol{\Theta})$. The entry at the $k$ th row and the $l$ th colmn of $\mathbf{S}, S_{k l}(\Theta)$ in (21), is the entry at the $k$ th row and the $l$ th colmn 

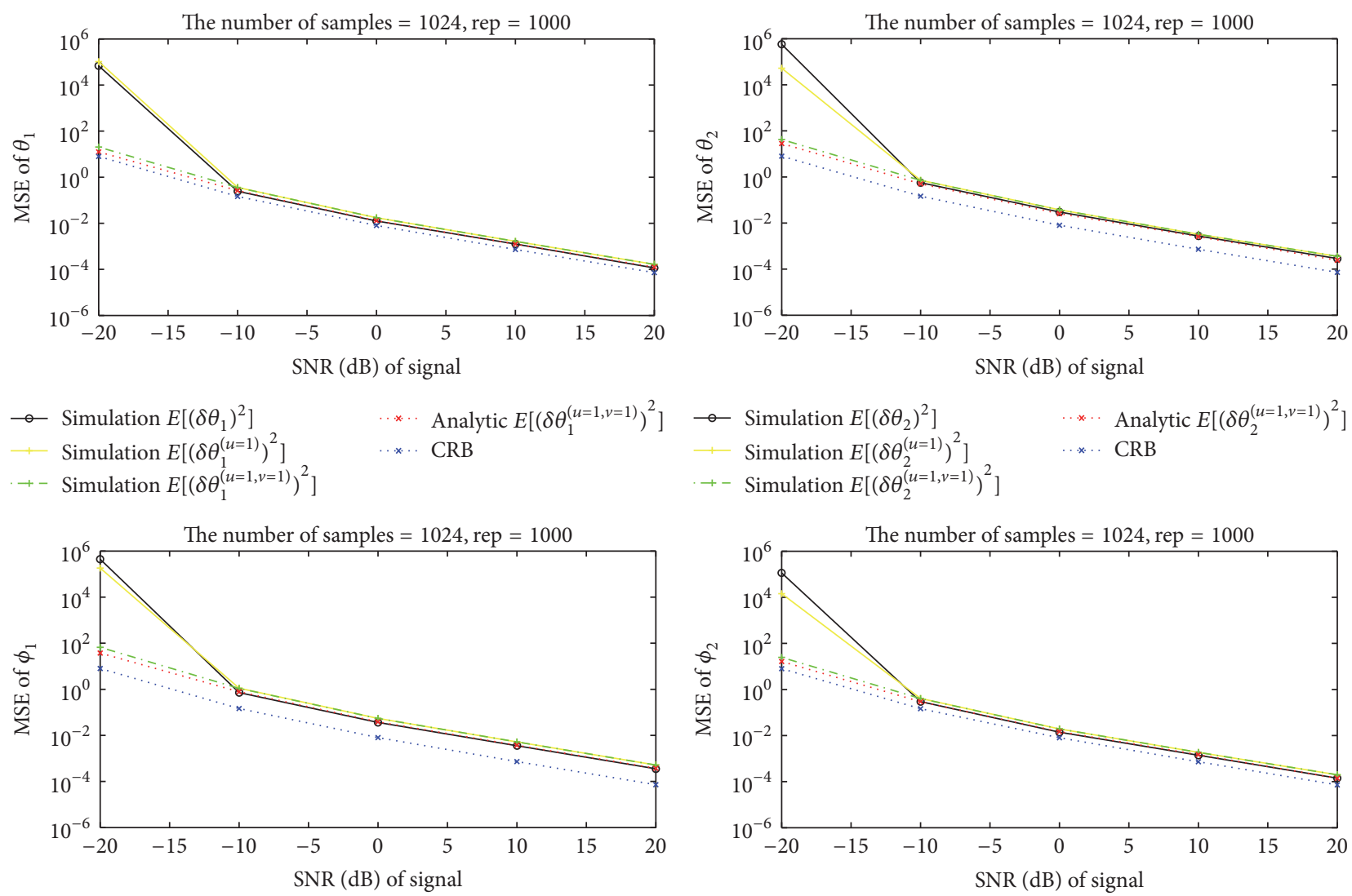

$E\left[\left(\delta \theta_{2},-1,\right)^{2}\right]$

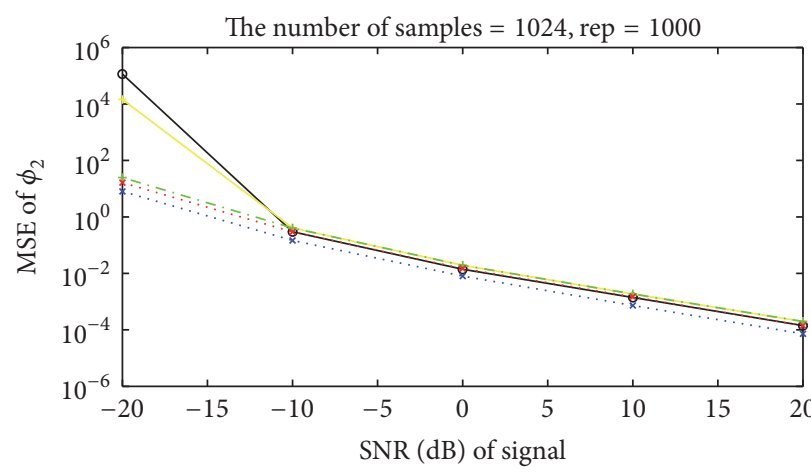

$\begin{array}{llll}\rightarrow & \text { Simulation } E\left[\left(\delta \phi_{1}\right)^{2}\right] & \cdots * \cdots & \text { Analytic } E\left[\left(\delta \phi_{1}^{(u=1, v=1)}\right)^{2}\right] \\ -+ & \text { Simulation } E\left[\left(\delta \phi_{1}^{(u=1)}\right)^{2}\right] & \cdots * \cdots & \text { CRB } \\ \cdots+ & \text { Simulation } E\left[\left(\delta \phi_{1}^{(u=1, v=1)}\right)^{2}\right] & & \end{array}$

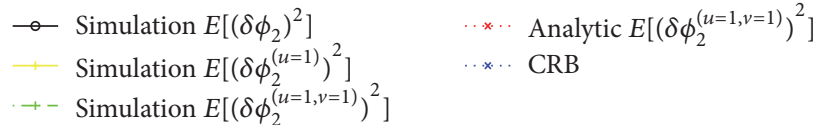

Figure 4: MSE of $\theta_{1}, \theta_{2}, \phi_{1}$, and $\phi_{2}$ for SNR $-20 \mathrm{~dB}$ to $20 \mathrm{~dB}$ (the number of samples $=2^{10}$ ).

of the adjoint matrix of $\mathbf{A}^{\mathrm{H}}(\boldsymbol{\Theta}) \mathbf{A}(\boldsymbol{\Theta})$. Note also that $\mathbf{P}_{\mathbf{A}(\Theta)}=$ $\mathbf{A}(\Theta)\left(\mathbf{A}^{\mathbf{H}}(\Theta) \mathbf{A}(\Theta)\right)^{-1} \mathbf{A}^{\mathbf{H}}(\Theta)$ is $M \times M$ matrix, where $M$ is the number of antenna elements. Therefore, the number of rows and the number of columns of $\mathbf{P}_{\mathbf{A}(\Theta)}$ are not dependent on the number of incident signals, $N$. Therefore, simple modification of the scheme presented in this paper results in more general scheme, which can be used irrespective of the number of incident signals.

Equation (7) of this manuscript is valid only for the uniform circular array. For different array structure, (7) should be modified appropriately. In that case, we only have to simply modify

(i) the expressions associated with the first derivatives of (7), which are (D.1),

(ii) the expressions associated with the second derivatives of (7), which are (K.1).

Simply modifying (D.1) and (K.1) for specific array structure is not so hard. Therefore, although we presented the formulation for the UCA structure, it can be very easily extended to any array structure.

\section{Appendix}

\section{A. The First-Order Derivatives of $D(\Theta)$ with respect to $\theta_{1}, \theta_{2}, \phi_{1}$, and $\phi_{2}$}

For use in (27), Appendices F and G, the first-order partial derivatives of $D(\Theta)$ in $(20)$ with respect to each argument are expressed as

$$
\begin{aligned}
& \frac{\partial}{\partial \theta_{1}} D(\Theta) \\
& \quad=-\sum_{k=1}^{M} \sum_{k^{\prime}=1}^{M}\left(\left(\frac{\partial}{\partial \theta_{1}}\left(a_{k, 1}\right)^{*}\right) a_{k, 2} a_{k^{\prime}, 1}\left(a_{k^{\prime}, 2}\right)^{*}\right. \\
& \left.\quad+\left(a_{k, 1}\right)^{*} a_{k, 2}\left(\frac{\partial}{\partial \theta_{1}} a_{k^{\prime}, 1}\right)\left(a_{k^{\prime}, 2}\right)^{*}\right), \\
& \frac{\partial}{\partial \theta_{2}} D(\Theta) \\
& \quad=-\sum_{k=1}^{M} \sum_{k^{\prime}=1}^{M}\left(\left(a_{k, 1}\right)^{*}\left(\frac{\partial}{\partial \theta_{2}} a_{k, 2}\right) a_{k^{\prime}, 1}\left(a_{k^{\prime}, 2}\right)^{*}\right.
\end{aligned}
$$



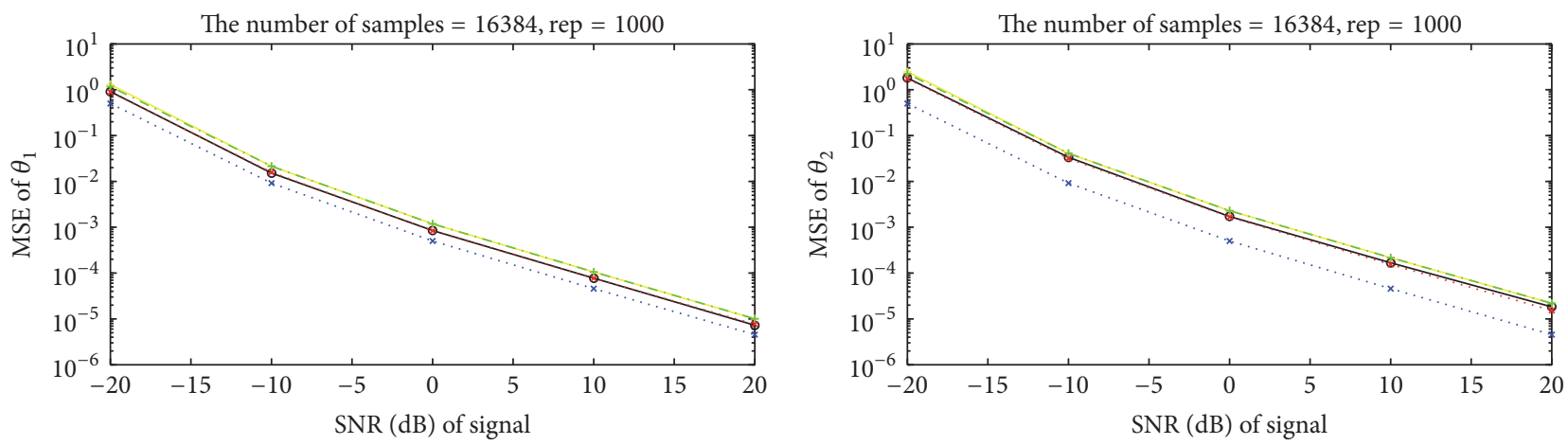

$\begin{array}{llll}\rightarrow & \text { Simulation } E\left[\left(\delta \theta_{1}\right)^{2}\right] & \cdots * \cdots & \text { Analytic } E\left[\left(\delta \theta_{1}^{(u=1, v=1)}\right)^{2}\right] \\ \rightarrow & \text { Simulation } E\left[\left(\delta \theta_{1}^{(u=1)}\right)^{2}\right] & \cdots * \cdots & \text { CRLB } \\ \cdots & \text { Simulation } E\left[\left(\delta \theta_{1}^{(u=1, v=1)}\right)^{2}\right] & & \end{array}$
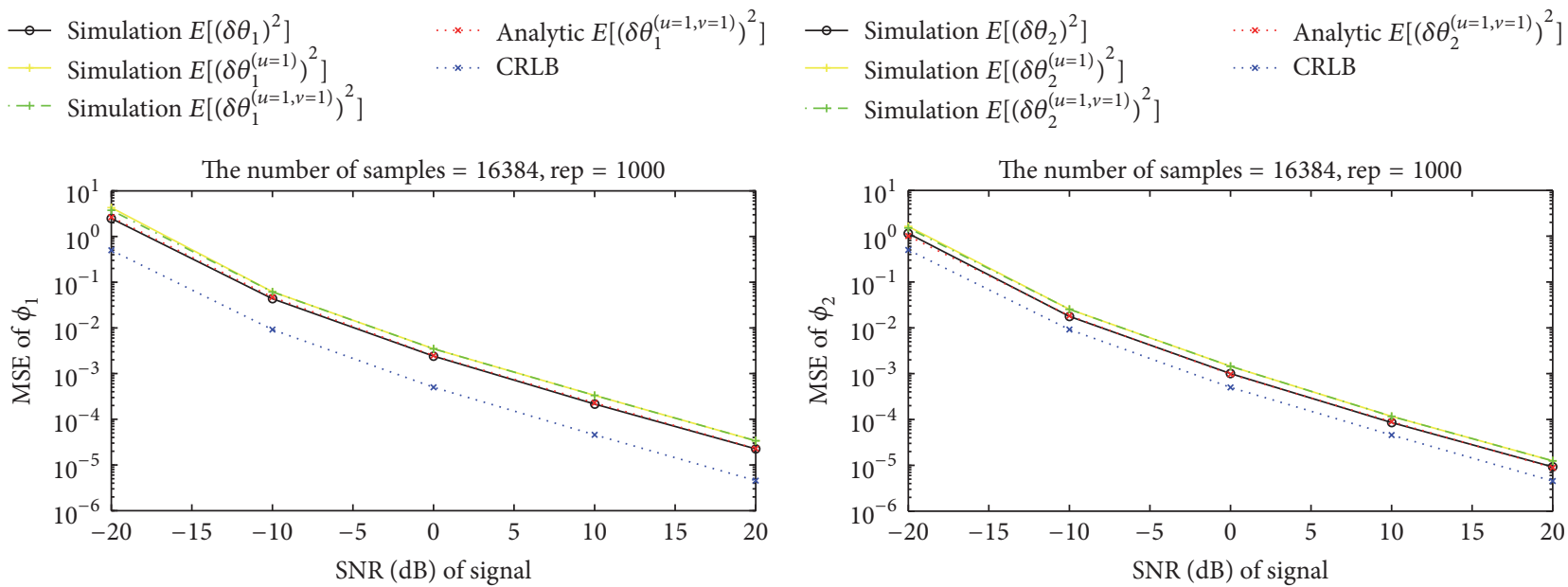
$\rightarrow$ Simulation $E\left[\left(\delta \phi_{2}\right)^{2}\right]$
Simulation $E\left[\left(\delta \phi_{2}^{(u=1)}\right)^{2}\right]$
* Analytic $E\left[\left(\delta \phi_{2}^{(u=1, v=1)}\right)^{2}\right]$
$\rightarrow-$ Simulation $E\left[\left(\delta \phi_{2}^{(u=1, v=1)}\right)^{2}\right.$

Figure 5: MSE of $\theta_{1}, \theta_{2}, \phi_{1}$, and $\phi_{2}$ for SNR $-20 \mathrm{~dB}$ to $20 \mathrm{~dB}$ (the number of samples $=2^{14}$ ).
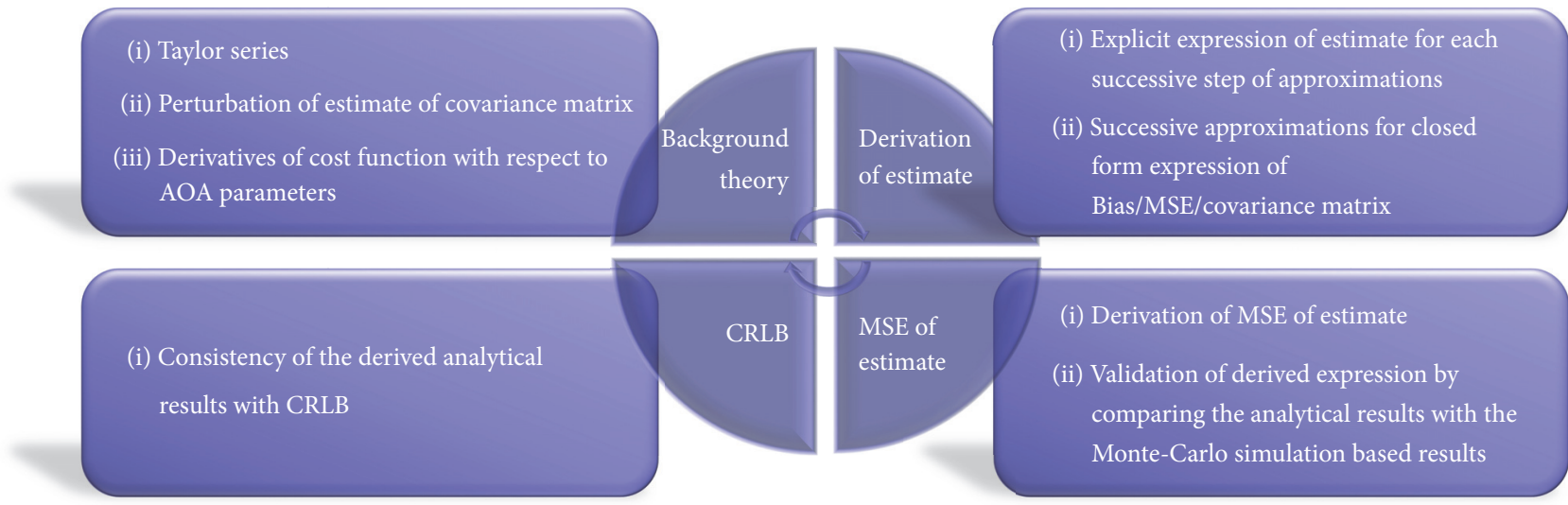

FIGURE 6: Overview of the proposed analytical performance analysis of ML algorithm.

$$
\begin{aligned}
& \left.+\left(a_{k, 1}\right)^{*} a_{k, 2} a_{k^{\prime}, 1}\left(\frac{\partial}{\partial \theta_{2}}\left(a_{k^{\prime}, 2}\right)^{*}\right)\right), \\
& \frac{\partial}{\partial \phi_{1}} D(\Theta)
\end{aligned}
$$

$$
\begin{aligned}
& =-\sum_{k=1}^{M} \sum_{k^{\prime}=1}^{M}\left(\left(\frac{\partial}{\partial \phi_{1}}\left(a_{k, 1}\right)^{*}\right) a_{k, 2} a_{k^{\prime}, 1}\left(a_{k^{\prime}, 2}\right)^{*}\right. \\
& \left.+\left(a_{k, 1}\right)^{*} a_{k, 2}\left(\frac{\partial}{\partial \phi_{1}} a_{k^{\prime}, 1}\right)\left(a_{k^{\prime}, 2}\right)^{*}\right),
\end{aligned}
$$




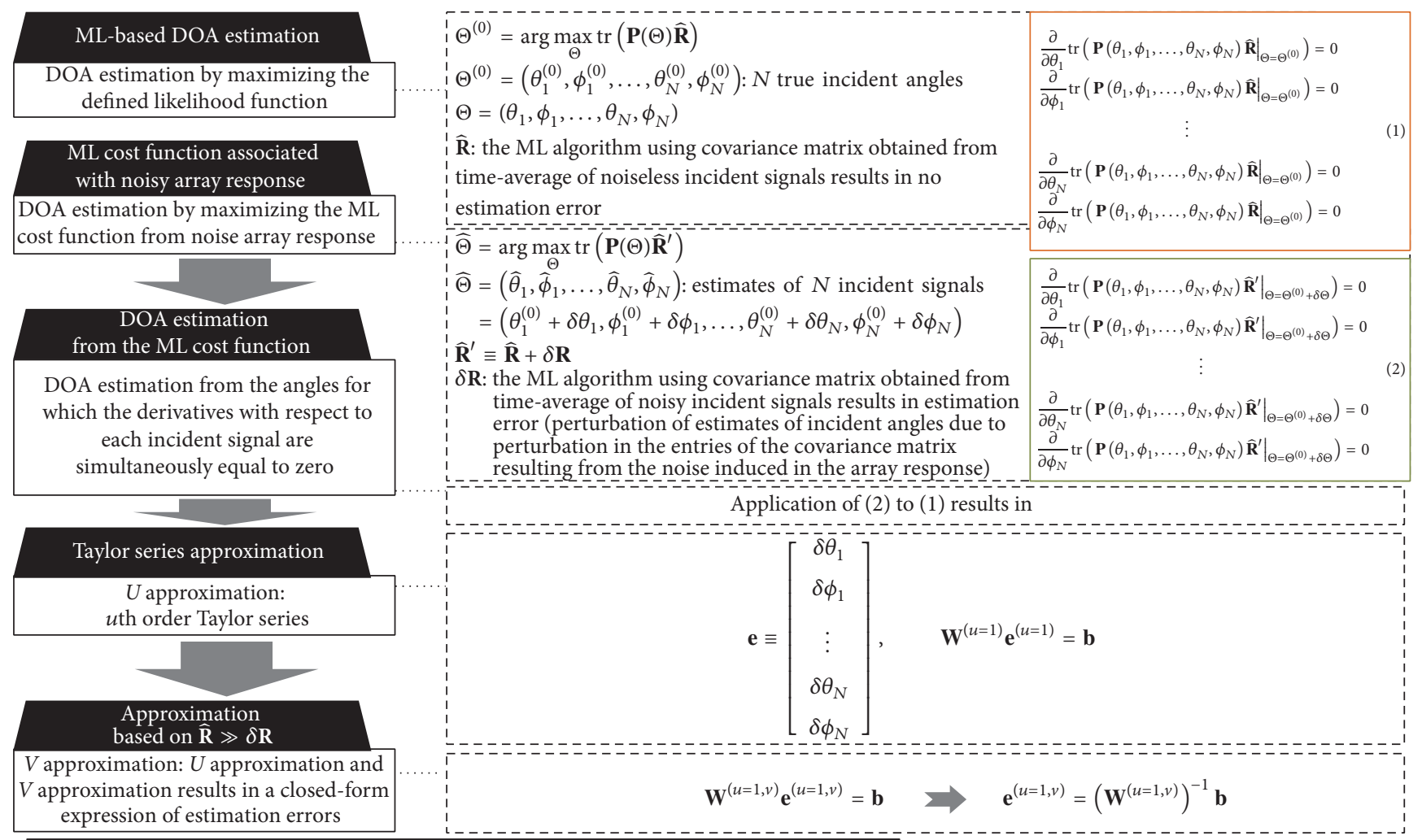

Approximation used for derivation of analytic expression of MSE of estimate

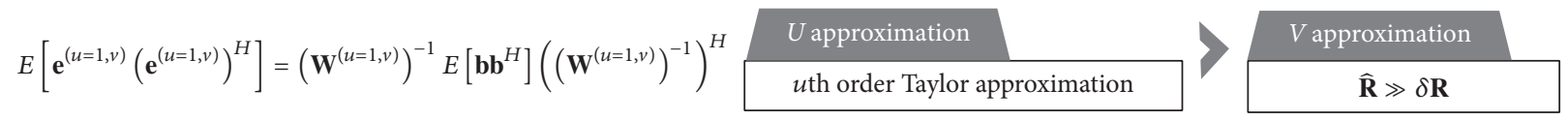

FIGURE 7: Summary of performance analysis of ML DOA estimation algorithm.

$$
\begin{aligned}
& \frac{\partial}{\partial \phi_{2}} D(\Theta)=\sum_{k=1}^{M} \sum_{k^{\prime}=1}^{M}\left(\left(a_{k, 1}\right)^{*}\left(\frac{\partial}{\partial \phi_{2}} a_{k, 2}\right) a_{k^{\prime}, 1}\left(a_{k^{\prime}, 2}\right)^{*}\right. \\
& \left.\quad+\left(a_{k, 1}\right)^{*} a_{k, 2} a_{k^{\prime}, 1}\left(\frac{\partial}{\partial \phi_{2}}\left(a_{k^{\prime}, 2}\right)^{*}\right)\right) .
\end{aligned}
$$

\section{B. The First-Order Derivatives of $S_{k l}(\Theta)$ with respect to $\theta_{1}, \theta_{2}, \phi_{1}$, and $\phi_{2}$}

For use in (27), Appendices E and G, the first-order partial derivatives of $S_{k l}$ in (23) with respect to each argument are expressed as

$$
\begin{aligned}
& \frac{\partial}{\partial \theta_{1}} S_{k l}(\Theta)=\left(\frac{\partial}{\partial \theta_{1}}\left(a_{l, 1}\right)^{*}\right)\left(a_{k, 1} M-a_{k, 2}(Q(\Theta))^{*}\right) \\
& +\left(a_{l, 1}\right)^{*}\left(\left(\frac{\partial}{\partial \theta_{1}} a_{k, 1}\right) M-a_{k, 2}\left(\frac{\partial}{\partial \theta_{1}}(Q(\Theta))^{*}\right)\right) \\
& +\left(a_{l, 2}\right)^{*}\left(-\left(\frac{\partial}{\partial \theta_{1}} a_{k, 1}\right) Q(\Theta)\right.
\end{aligned}
$$

$$
\begin{aligned}
& \left.-a_{k, 1}\left(\frac{\partial}{\partial \theta_{1}} Q(\Theta)\right)\right) \\
& \frac{\partial}{\partial \theta_{2}} S_{k l}(\Theta)=\left(a_{l, 1}\right)^{*}\left(-\left(\frac{\partial}{\partial \theta_{2}} a_{k, 2}\right)(Q(\Theta))^{*}\right. \\
& \left.-a_{k, 2}\left(\frac{\partial}{\partial \theta_{2}}(Q(\Theta))^{*}\right)\right)+\left(\frac{\partial}{\partial \theta_{2}}\left(a_{l, 2}\right)^{*}\right)\left(a_{k, 2} M\right. \\
& \left.-a_{k, 1} Q(\Theta)\right)+\left(a_{l, 2}\right)^{*}\left(\left(\frac{\partial}{\partial \theta_{2}} a_{k, 2}\right) M\right. \\
& \left.\quad-a_{k, 1}\left(\frac{\partial}{\partial \theta_{2}} Q(\Theta)\right)\right), \\
& \frac{\partial}{\partial \phi_{1}} S_{k l}(\Theta)=\left(\frac{\partial}{\partial \phi_{1}}\left(a_{l, 1}\right)^{*}\right)\left(a_{k, 1} M-a_{k, 2}(Q(\Theta))^{*}\right) \\
& +\left(a_{l, 1}\right)^{*}\left(\left(\frac{\partial}{\partial \phi_{1}} a_{k, 1}\right) M-a_{k, 2}\left(\frac{\partial}{\partial \phi_{1}}(Q(\Theta))^{*}\right)\right) \\
& \quad+\left(a_{l, 2}\right)^{*}\left(-\left(\frac{\partial}{\partial \phi_{1}} a_{k, 1}\right) Q(\Theta)\right. \\
& \left.\quad-a_{k, 1}\left(\frac{\partial}{\partial \phi_{1}} Q(\Theta)\right)\right),
\end{aligned}
$$




$$
\begin{aligned}
& \frac{\partial}{\partial \phi_{2}} S_{k l}(\Theta)=\left(a_{l, 1}\right)^{*}\left(-\left(\frac{\partial}{\partial \phi_{2}} a_{k, 2}\right)(Q(\Theta))^{*}\right. \\
& \left.-a_{k, 2}\left(\frac{\partial}{\partial \phi_{2}}(Q(\Theta))^{*}\right)\right)+\left(\frac{\partial}{\partial \phi_{2}}\left(a_{l, 2}\right)^{*}\right)\left(a_{k, 2} M\right. \\
& \left.\quad-a_{k, 1} Q(\Theta)\right)+\left(a_{l, 2}\right)^{*}\left(\left(\frac{\partial}{\partial \phi_{2}} a_{k, 2}\right) M\right. \\
& \left.\quad-a_{k, 1}\left(\frac{\partial}{\partial \phi_{2}} Q(\Theta)\right)\right) .
\end{aligned}
$$

\section{The First-Order Derivatives of $Q(\Theta)$ with respect to $\theta_{1}, \theta_{2}, \phi_{1}$, and $\phi_{2}$}

For use in Appendices B and L, the first-order partial derivatives of $Q(\Theta)$ in (17) with respect to each argument are expressed as

$$
\begin{aligned}
\frac{\partial}{\partial \theta_{1}} Q(\Theta) & =\sum_{k=1}^{M}\left(\left(\frac{\partial}{\partial \theta_{1}}\left(a_{k, 1}\right)^{*}\right) a_{k, 2}\right), \\
\frac{\partial}{\partial \theta_{2}} Q(\Theta) & =\sum_{k=1}^{M}\left(\left(a_{k, 1}\right)^{*}\left(\frac{\partial}{\partial \theta_{2}} a_{k, 2}\right)\right), \\
\frac{\partial}{\partial \phi_{1}} Q(\Theta) & =\sum_{k=1}^{M}\left(\left(\frac{\partial}{\partial \phi_{1}}\left(a_{k, 1}\right)^{*}\right) a_{k, 2}\right), \\
\frac{\partial}{\partial \phi_{2}} Q(\Theta) & =\sum_{k=1}^{M}\left(\left(a_{k, 1}\right)^{*}\left(\frac{\partial}{\partial \phi_{2}} a_{k, 2}\right)\right) .
\end{aligned}
$$

\section{The First-Order Derivatives of $a_{m}\left(\theta_{n}, \phi_{n}\right)$ with respect to $\theta_{n}$ and $\phi_{n}$}

For use in Appendices A, B, C, E, F, I, J, K, and L, the firstorder partial derivatives of $a_{m}\left(\theta_{n}, \phi_{n}\right)$ in (18) with respect to each argument are expressed as

$$
\begin{aligned}
& \frac{\partial}{\partial \theta_{n}} a_{m}\left(\theta_{n}, \phi_{n}\right) \\
& =-j \frac{2 \pi r}{\lambda} \sin \left(\theta_{n}-\frac{2 \pi}{M}(m-1)\right) \cos \phi_{n} a_{m}\left(\theta_{n}, \phi_{n}\right), \\
& \frac{\partial}{\partial \phi_{n}} a_{m}\left(\theta_{n}, \phi_{n}\right) \\
& =-j \frac{2 \pi r}{\lambda} \cos \left(\theta_{n}-\frac{2 \pi}{M}(m-1)\right) \sin \phi_{n} a_{m}\left(\theta_{n}, \phi_{n}\right) .
\end{aligned}
$$

\section{E. The Second-Order Partial Derivatives of $S_{k l}(\Theta)$}

For use in Appendix G, the second partial derivatives of $S_{k l}(\Theta)$ in (23) with respect to each argument are expressed as

$$
\begin{aligned}
& \frac{\partial^{2}}{\partial \theta_{1}^{2}} S_{k l}(\Theta)=\left(\frac{\partial^{2}}{\partial \theta_{1}^{2}}\left(a_{l, 1}\right)^{*}\right)\left(a_{k, 1} M-a_{k, 2}(Q(\Theta))^{*}\right) \\
& +\left(a_{l, 1}\right)^{*}\left(\left(\frac{\partial^{2}}{\partial \theta_{1}^{2}} a_{k, 1}\right) M-a_{k, 2}\left(\frac{\partial^{2}}{\partial \theta_{1}^{2}}(Q(\Theta))^{*}\right)\right) \\
& +\left(\frac{\partial}{\partial \theta_{1}}\left(a_{l, 1}\right)^{*}\right)\left(\left(\frac{\partial}{\partial \theta_{1}} a_{k, 1}\right) M\right. \\
& \left.-a_{k, 2}\left(\frac{\partial}{\partial \theta_{1}}(Q(\Theta))^{*}\right)\right)+\left(\frac{\partial}{\partial \theta_{1}}\left(a_{l, 1}\right)^{*}\right) \\
& \cdot\left(\left(\frac{\partial}{\partial \theta_{1}} a_{k, 1}\right) M-a_{k, 2}\left(\frac{\partial}{\partial \theta_{1}}(Q(\Theta))^{*}\right)\right) \\
& +\left(a_{l, 2}\right)^{*}\left(-\left(\frac{\partial^{2}}{\partial \theta_{1}^{2}} a_{k, 1}\right) Q(\Theta)\right. \\
& \left.-\left(\frac{\partial}{\partial \theta_{1}} a_{k, 1}\right)\left(\frac{\partial}{\partial \theta_{1}} Q(\Theta)\right)\right)+\left(a_{l, 2}\right)^{*} \\
& \cdot\left(-\left(\frac{\partial}{\partial \theta_{1}} a_{k, 1}\right)\left(\frac{\partial}{\partial \theta_{1}} Q(\Theta)\right)\right. \\
& \left.-a_{k, 1}\left(\frac{\partial^{2}}{\partial \theta_{1}^{2}} Q(\Theta)\right)\right) \text {, } \\
& \frac{\partial}{\partial \theta_{2}} \frac{\partial}{\partial \theta_{1}} S_{k l}(\Theta)=\left(\frac{\partial}{\partial \theta_{1}}\left(a_{l, 1}\right)^{*}\right) \\
& \cdot\left(-\left(\frac{\partial}{\partial \theta_{2}} a_{k, 2}\right)(Q(\Theta))^{*}-a_{k, 2}\left(\frac{\partial}{\partial \theta_{2}}(Q(\Theta))^{*}\right)\right) \\
& +\left(a_{l, 1}\right)^{*}\left(\left(\frac{\partial}{\partial \theta_{2}} a_{k, 2}\right)\left(\frac{\partial}{\partial \theta_{1}}(Q(\Theta))^{*}\right)\right. \\
& \left.-a_{k, 2}\left(\frac{\partial}{\partial \theta_{2}} \frac{\partial}{\partial \theta_{1}}(Q(\Theta))^{*}\right)\right)+\left(\frac{\partial}{\partial \theta_{2}}\left(a_{l, 2}\right)^{*}\right) \\
& \cdot\left(-\left(\frac{\partial}{\partial \theta_{1}} a_{k, 1}\right) Q(\Theta)-a_{k, 1}\left(\frac{\partial}{\partial \theta_{1}} Q(\Theta)\right)\right) \\
& +\left(a_{l, 2}\right)^{*}\left(-\left(\frac{\partial}{\partial \theta_{1}} a_{k, 1}\right)\left(\frac{\partial}{\partial \theta_{2}} Q(\Theta)\right)\right. \\
& \left.-a_{k, 1}\left(\frac{\partial}{\partial \theta_{2}} \frac{\partial}{\partial \theta_{1}} Q(\Theta)\right)\right), \\
& \frac{\partial}{\partial \phi_{1}} \frac{\partial}{\partial \theta_{1}} S_{k l}(\Theta)=\left(\frac{\partial}{\partial \phi_{1}} \frac{\partial}{\partial \theta_{1}}\left(a_{l, 1}\right)^{*}\right)\left(a_{k, 1} M\right. \\
& \left.-a_{k, 2}(Q(\Theta))^{*}\right)+\left(\frac{\partial}{\partial \theta_{1}}\left(a_{l, 1}\right)^{*}\right)\left(\left(\frac{\partial}{\partial \phi_{1}} a_{k, 1}\right) M\right. \\
& \left.-a_{k, 2}\left(\frac{\partial}{\partial \phi_{1}}(Q(\Theta))^{*}\right)\right)+\left(\frac{\partial}{\partial \phi_{1}}\left(a_{l, 1}\right)^{*}\right) \\
& \cdot\left(\left(\frac{\partial}{\partial \theta_{1}} a_{k, 1}\right) M-a_{k, 2}\left(\frac{\partial}{\partial \theta_{1}}(Q(\Theta))^{*}\right)\right) \\
& +\left(a_{l, 1}\right)^{*}\left(\left(\frac{\partial}{\partial \phi_{1}} \frac{\partial}{\partial \theta_{1}} a_{k, 1}\right) M\right.
\end{aligned}
$$




$$
\begin{aligned}
& \left.-a_{k, 2}\left(\frac{\partial}{\partial \phi_{1}} \frac{\partial}{\partial \theta_{1}}(Q(\Theta))^{*}\right)\right)+\left(a_{l, 2}\right)^{*} \\
& \cdot\left(-\left(\frac{\partial}{\partial \phi_{1}} \frac{\partial}{\partial \theta_{1}} a_{k, 1}\right) Q(\Theta)\right. \\
& \left.-\left(\frac{\partial}{\partial \theta_{1}} a_{k, 1}\right)\left(\frac{\partial}{\partial \phi_{1}} Q(\Theta)\right)\right)+\left(a_{l, 2}\right)^{*} \\
& \cdot\left(-\left(\frac{\partial}{\partial \phi_{1}} a_{k, 1}\right)\left(\frac{\partial}{\partial \theta_{1}} Q(\Theta)\right)\right. \\
& \left.-a_{k, 1}\left(\frac{\partial}{\partial \phi_{1}} \frac{\partial}{\partial \theta_{1}} Q(\Theta)\right)\right), \\
& \frac{\partial}{\partial \phi_{2}} \frac{\partial}{\partial \theta_{1}} S_{k l}(\Theta)=\left(\frac{\partial}{\partial \theta_{1}}\left(a_{l, 1}\right)^{*}\right) \\
& \cdot\left(-\left(\frac{\partial}{\partial \phi_{2}} a_{k, 2}\right)(Q(\Theta))^{*}\right. \\
& \left.-a_{k, 2}\left(\frac{\partial}{\partial \phi_{2}}(Q(\Theta))^{*}\right)\right)+\left(a_{l, 1}\right)^{*} \\
& \cdot\left(-\left(\frac{\partial}{\partial \phi_{2}} a_{k, 2}\right)\left(\frac{\partial}{\partial \theta_{1}}(Q(\Theta))^{*}\right)\right. \\
& \left.-a_{k, 2}\left(\frac{\partial}{\partial \phi_{2}} \frac{\partial}{\partial \theta_{1}}(Q(\Theta))^{*}\right)\right)+\left(\frac{\partial}{\partial \phi_{2}}\left(a_{l, 2}\right)^{*}\right) \\
& \cdot\left(-\left(\frac{\partial}{\partial \theta_{1}} a_{k, 1}\right) Q(\Theta)-a_{k, 1}\left(\frac{\partial}{\partial \theta_{1}} Q(\Theta)\right)\right) \\
& +\left(a_{l, 2}\right)^{*}\left(-\left(\frac{\partial}{\partial \theta_{1}} a_{k, 1}\right)\left(\frac{\partial}{\partial \phi_{2}} Q(\Theta)\right)\right. \\
& \left.-a_{k, 1}\left(\frac{\partial}{\partial \phi_{2}} \frac{\partial}{\partial \theta_{1}} Q(\Theta)\right)\right), \\
& \frac{\partial^{2}}{\partial \theta_{2}^{2}} S_{k l}(\Theta)=\left(a_{l, 1}\right)^{*}\left(-\left(\frac{\partial^{2}}{\partial \theta_{2}^{2}} a_{k, 2}\right)(Q(\Theta))^{*}\right. \\
& \left.-\left(\frac{\partial}{\partial \theta_{2}} a_{k, 2}\right)\left(\frac{\partial}{\partial \theta_{2}}(Q(\Theta))^{*}\right)\right)+\left(a_{l, 1}\right)^{*} \\
& \cdot\left(-\left(\frac{\partial}{\partial \theta_{2}} a_{k, 2}\right)\left(\frac{\partial}{\partial \theta_{2}}(Q(\Theta))^{*}\right)\right. \\
& \left.-a_{k, 2}\left(\frac{\partial^{2}}{\partial \theta_{2}^{2}}(Q(\Theta))^{*}\right)\right)+\left(\frac{\partial^{2}}{\partial \theta_{2}^{2}}\left(a_{l, 2}\right)^{*}\right)\left(a_{k, 2} M\right. \\
& \left.-a_{k, 1} Q(\Theta)\right)+\left(\frac{\partial}{\partial \theta_{2}}\left(a_{l, 2}\right)^{*}\right)\left(\left(\frac{\partial}{\partial \theta_{2}} a_{k, 2}\right) M\right. \\
& \left.-a_{k, 1}\left(\frac{\partial}{\partial \theta_{2}} Q(\Theta)\right)\right)+\left(\frac{\partial}{\partial \theta_{2}}\left(a_{l, 2}\right)^{*}\right) \\
& \cdot\left(\left(\frac{\partial}{\partial \theta_{2}} a_{k, 2}\right) M-a_{k, 1}\left(\frac{\partial}{\partial \theta_{2}} Q(\Theta)\right)\right)+\left(a_{l, 2}\right)^{*} \\
& \cdot\left(\left(\frac{\partial^{2}}{\partial \theta_{2}^{2}} a_{k, 2}\right) M-a_{k, 1}\left(\frac{\partial^{2}}{\partial \theta_{2}^{2}} Q(\Theta)\right)\right), \\
& \frac{\partial}{\partial \phi_{1}} \frac{\partial}{\partial \theta_{2}} S_{k l}(\Theta)=\left(\frac{\partial}{\partial \phi_{1}}\left(a_{l, 1}\right)^{*}\right) \\
& \cdot\left(-\left(\frac{\partial}{\partial \theta_{2}} a_{k, 2}\right)(Q(\Theta))^{*}-a_{k, 2}\left(\frac{\partial}{\partial \theta_{2}}(Q(\Theta))^{*}\right)\right) \\
& +\left(a_{l, 1}\right)^{*}\left(-\left(\frac{\partial}{\partial \theta_{2}} a_{k, 2}\right)\left(\frac{\partial}{\partial \phi_{1}}(Q(\Theta))^{*}\right)\right. \\
& \left.-a_{k, 2}\left(\frac{\partial}{\partial \phi_{1}} \frac{\partial}{\partial \theta_{2}} Q^{*}(\Theta)\right)\right)+\left(\frac{\partial}{\partial \theta_{2}}\left(a_{l, 2}\right)^{*}\right) \\
& \cdot\left(-\left(\frac{\partial}{\partial \phi_{1}} a_{k, 1}\right) Q(\Theta)-a_{k, 1}\left(\frac{\partial}{\partial \phi_{1}} Q(\Theta)\right)\right) \\
& +\left(a_{l, 2}\right)^{*}\left(\left(\frac{\partial}{\partial \phi_{1}} a_{k, 1}\right)\left(\frac{\partial}{\partial \theta_{2}} Q(\Theta)\right)\right. \\
& \left.-a_{k, 1}\left(\frac{\partial}{\partial \phi_{1}} \frac{\partial}{\partial \theta_{2}} Q(\Theta)\right)\right), \\
& \frac{\partial}{\partial \phi_{2}} \frac{\partial}{\partial \theta_{2}} S_{k l}(\Theta)=\left(a_{l, 1}\right)^{*}\left(-\left(\frac{\partial}{\partial \phi_{2}} \frac{\partial}{\partial \theta_{2}} a_{k, 2}\right)(Q(\Theta))^{*}\right. \\
& \left.-\left(\frac{\partial}{\partial \theta_{2}} a_{k, 2}\right)\left(\frac{\partial}{\partial \phi_{2}}(Q(\Theta))^{*}\right)\right)+\left(a_{l, 1}\right)^{*} \\
& \cdot\left(-\left(\frac{\partial}{\partial \phi_{2}} a_{k, 2}\right)\left(\frac{\partial}{\partial \theta_{2}}(Q(\Theta))^{*}\right)\right. \\
& \left.-a_{k, 2}\left(\frac{\partial}{\partial \phi_{2}} \frac{\partial}{\partial \theta_{2}}(Q(\Theta))^{*}\right)\right)+\left(\frac{\partial}{\partial \phi_{2}} \frac{\partial}{\partial \theta_{2}}\left(a_{l, 2}\right)^{*}\right) \\
& \cdot\left(a_{k, 2} M-a_{k, 1} Q(\Theta)\right)+\left(\frac{\partial}{\partial \theta_{2}}\left(a_{l, 2}\right)^{*}\right) \\
& \cdot\left(\left(\frac{\partial}{\partial \phi_{2}} a_{k, 2}\right) M-a_{k, 1}\left(\frac{\partial}{\partial \phi_{2}} Q(\Theta)\right)\right) \\
& +\left(\frac{\partial}{\partial \phi_{2}}\left(a_{l, 2}\right)^{*}\right)\left(\left(\frac{\partial}{\partial \theta_{2}} a_{k, 2}\right) M\right. \\
& \left.-a_{k, 1}\left(\frac{\partial}{\partial \theta_{2}} Q(\Theta)\right)\right)+\left(a_{l, 2}\right)^{*}\left(\left(\frac{\partial}{\partial \phi_{2}} \frac{\partial}{\partial \theta_{2}} a_{k, 2}\right) M\right. \\
& \left.-a_{k, 1}\left(\frac{\partial}{\partial \phi_{2}} \frac{\partial}{\partial \theta_{2}} Q(\Theta)\right)\right), \\
& \frac{\partial^{2}}{\partial \phi_{1}^{2}} S_{k l}(\Theta)=\left(\frac{\partial^{2}}{\partial \phi_{1}^{2}}\left(a_{l, 1}\right)^{*}\right)\left(a_{k, 1} M-a_{k, 2}(Q(\Theta))\right) \\
& +\left(\frac{\partial}{\partial \phi_{1}}\left(a_{l, 1}\right)^{*}\right)\left(\left(\frac{\partial}{\partial \phi_{1}} a_{k, 1}\right) M\right. \\
& \left.-a_{k, 2}\left(\frac{\partial}{\partial \phi_{1}}(Q(\Theta))^{*}\right)\right)+\left(\frac{\partial}{\partial \phi_{1}}\left(a_{l, 1}\right)^{*}\right) \\
& \cdot\left(\left(\frac{\partial}{\partial \phi_{1}} a_{k, 1}\right) M-a_{k, 2}\left(\frac{\partial}{\partial \phi_{1}}(Q(\Theta))^{*}\right)\right) \\
& +\left(a_{l, 1}\right)^{*}\left(\left(\frac{\partial^{2}}{\partial \phi_{1}^{2}} a_{k, 1}\right) M-a_{k, 2}\left(\frac{\partial^{2}}{\partial \phi_{1}^{2}}(Q(\Theta))^{*}\right)\right)
\end{aligned}
$$




$$
\begin{aligned}
& +\left(a_{l, 2}\right)^{*}\left(-\left(\frac{\partial^{2}}{\partial \phi_{1}^{2}} a_{k, 1}\right) Q(\Theta)\right. \\
& \left.-\left(\frac{\partial}{\partial \phi_{1}} a_{k, 1}\right)\left(\frac{\partial}{\partial \phi_{1}} Q(\Theta)\right)\right)+\left(a_{l, 2}\right)^{*} \\
& \cdot\left(-\left(\frac{\partial^{2}}{\partial \phi_{1}^{2}} a_{k, 1}\right) Q(\Theta)\right. \\
& \left.-\left(\frac{\partial}{\partial \phi_{1}} a_{k, 1}\right)\left(\frac{\partial}{\partial \phi_{1}} Q(\Theta)\right)\right), \\
& \frac{\partial}{\partial \phi_{2}} \frac{\partial}{\partial \phi_{1}} S_{k l}(\Theta)=\left(\frac{\partial}{\partial \phi_{1}}\left(a_{l, 1}\right)^{*}\right) \\
& \cdot\left(-\left(\frac{\partial}{\partial \phi_{2}} a_{k, 2}\right)(Q(\Theta))^{*}\right. \\
& \left.-a_{k, 2}\left(\frac{\partial}{\partial \phi_{2}}(Q(\Theta))^{*}\right)\right)+\left(a_{l, 1}\right)^{*} \\
& \cdot\left(-\left(\frac{\partial}{\partial \phi_{2}} a_{k, 2}\right)\left(\frac{\partial}{\partial \phi_{1}}(Q(\Theta))^{*}\right)\right. \\
& \left.-a_{k, 2}\left(\frac{\partial}{\partial \phi_{2}} \frac{\partial}{\partial \phi_{1}}(Q(\Theta))^{*}\right)\right)+\left(\frac{\partial}{\partial \phi_{2}}\left(a_{l, 2}\right)^{*}\right) \\
& \cdot\left(-\left(\frac{\partial}{\partial \phi_{1}} a_{k, 1}\right) Q(\Theta)-a_{k, 1}\left(\frac{\partial}{\partial \phi_{1}} Q(\Theta)\right)\right) \\
& +\left(a_{l, 2}\right)^{*}\left(-\left(\frac{\partial}{\partial \phi_{1}} a_{k, 1}\right)\left(\frac{\partial}{\partial \phi_{2}} Q(\Theta)\right)\right. \\
& \left.-a_{k, 1}\left(\frac{\partial}{\partial \phi_{2}} \frac{\partial}{\partial \phi_{1}} Q(\Theta)\right)\right), \\
& \frac{\partial^{2}}{\partial \phi_{2}^{2}} S_{k l}(\Theta)=\left(a_{l, 1}\right)^{*}\left(-\left(\frac{\partial^{2}}{\partial \phi_{2}^{2}} a_{k, 2}\right)(Q(\Theta))^{*}\right. \\
& \left.-\left(\frac{\partial}{\partial \phi_{2}} a_{k, 2}\right)\left(\frac{\partial}{\partial \phi_{2}}(Q(\Theta))^{*}\right)\right)+\left(a_{l, 1}\right)^{*} \\
& \cdot\left(-\left(\frac{\partial}{\partial \phi_{2}} a_{k, 2}\right)\left(\frac{\partial}{\partial \phi_{2}}(Q(\Theta))^{*}\right)\right. \\
& \left.-a_{k, 2}\left(\frac{\partial^{2}}{\partial \phi_{2}^{2}}(Q(\Theta))^{*}\right)\right)+\left(\frac{\partial^{2}}{\partial \phi_{2}^{2}}\left(a_{l, 2}\right)^{*}\right)\left(a_{k, 2} M\right. \\
& \left.-a_{k, 1} Q(\Theta)\right)+\left(\frac{\partial}{\partial \phi_{2}}\left(a_{l, 2}\right)^{*}\right)\left(\left(\frac{\partial}{\partial \phi_{2}} a_{k, 2}\right) M\right. \\
& \left.-a_{k, 1}\left(\frac{\partial}{\partial \phi_{2}} Q(\Theta)\right)\right)+\left(\frac{\partial}{\partial \phi_{2}}\left(a_{l, 2}\right)^{*}\right) \\
& \cdot\left(\left(\frac{\partial}{\partial \phi_{2}} a_{k, 2}\right) M-a_{k, 1}\left(\frac{\partial}{\partial \phi_{2}} Q(\Theta)\right)\right)+\left(a_{l, 2}\right)^{*} \\
& \cdot\left(\left(\frac{\partial^{2}}{\partial \phi_{2}^{2}} a_{k, 2}\right) M-a_{k, 1}\left(\frac{\partial^{2}}{\partial \phi_{2}^{2}} Q(\Theta)\right)\right) .
\end{aligned}
$$

\section{F. The Second-Order Partial Derivatives of $D(\Theta)$}

For use in Appendix G, the second-order partial derivatives of $D(\Theta)$ in $(20)$ with respect to each argument are expressed as

$$
\begin{aligned}
& \frac{\partial^{2}}{\partial \theta_{1}^{2}} D(\Theta)=-\sum_{k=1}^{M} \sum_{k^{\prime}=1}^{M}\left(\left(\frac{\partial^{2}}{\partial \theta_{1}^{2}}\left(a_{k, 1}\right)^{*}\right)\right. \\
& \cdot a_{k, 2} a_{k^{\prime}, 1}\left(a_{k^{\prime}, 2}\right)^{*}+\left(\frac{\partial}{\partial \theta_{1}}\left(a_{k, 1}\right)^{*}\right) a_{k, 2}\left(\frac{\partial}{\partial \theta_{1}} a_{k^{\prime}, 1}\right) \\
& \cdot\left(a_{k^{\prime}, 2}\right)^{*}+\left(\frac{\partial}{\partial \theta_{1}}\left(a_{k, 1}\right)^{*}\right) a_{k, 2}\left(\frac{\partial}{\partial \theta_{1}} a_{k^{\prime}, 1}\right)\left(a_{k^{\prime}, 2}\right)^{*} \\
& \left.+\left(a_{k, 1}\right)^{*} a_{k, 2}\left(\frac{\partial^{2}}{\partial \theta_{1}^{2}} a_{k^{\prime}, 1}\right)\left(a_{k^{\prime}, 2}\right)^{*}\right), \\
& \frac{\partial}{\partial \theta_{2}}\left(\frac{\partial}{\partial \theta_{1}} D(\Theta)\right)=-\sum_{k=1}^{M} \sum_{k^{\prime}=1}^{M}\left(\left(\frac{\partial}{\partial \theta_{1}}\left(a_{k, 1}\right)^{*}\right)\right. \\
& \cdot\left(\frac{\partial}{\partial \theta_{2}} a_{k, 2}\right) a_{k^{\prime}, 1}\left(a_{k^{\prime}, 2}\right)^{*}+\left(\frac{\partial}{\partial \theta_{1}}\left(a_{k, 1}\right)^{*}\right) \\
& \cdot a_{k, 2} a_{k^{\prime}, 1}\left(\frac{\partial}{\partial \theta_{2}}\left(a_{k^{\prime}, 2}\right)^{*}\right)+\left(a_{k, 1}\right)^{*}\left(\frac{\partial}{\partial \theta_{2}} a_{k, 2}\right) \\
& \cdot\left(\frac{\partial}{\partial \theta_{1}} a_{k^{\prime}, 1}\right)\left(a_{k^{\prime}, 2}\right)^{*}+\left(a_{k, 1}\right)^{*} a_{k, 2}\left(\frac{\partial}{\partial \theta_{1}} a_{k^{\prime}, 1}\right) \\
& \left.\cdot\left(\frac{\partial}{\partial \theta_{2}}\left(a_{k^{\prime}, 2}\right)^{*}\right)\right) \text {, } \\
& \frac{\partial}{\partial \phi_{1}}\left(\frac{\partial}{\partial \theta_{1}} D(\Theta)\right) \\
& =-\sum_{k=1}^{M} \sum_{k^{\prime}=1}^{M}\left(\left(\frac{\partial}{\partial \phi_{1}}\left(\frac{\partial}{\partial \theta_{1}}\left(a_{k, 1}\right)^{*}\right)\right)\right. \\
& \cdot a_{k, 2} a_{k^{\prime}, 1}\left(a_{k^{\prime}, 2}\right)^{*}+\left(\frac{\partial}{\partial \theta_{1}}\left(a_{k, 1}\right)^{*}\right) a_{k, 2}\left(\frac{\partial}{\partial \phi_{1}} a_{k^{\prime}, 1}\right) \\
& \cdot\left(a_{k^{\prime}, 2}\right)^{*}+\left(\frac{\partial}{\partial \phi_{1}}\left(a_{k, 1}\right)^{*}\right) a_{k, 2}\left(\frac{\partial}{\partial \theta_{1}} a_{k^{\prime}, 1}\right)\left(a_{k^{\prime}, 2}\right)^{*} \\
& \left.+\left(a_{k, 1}\right)^{*} a_{k, 2}\left(\frac{\partial}{\partial \phi_{1}}\left(\frac{\partial}{\partial \theta_{1}} a_{k^{\prime}, 1}\right)\right)\left(a_{k^{\prime}, 2}\right)^{*}\right), \\
& \frac{\partial}{\partial \phi_{2}}\left(\frac{\partial}{\partial \theta_{1}} D(\Theta)\right)=-\sum_{k=1}^{M} \sum_{k^{\prime}=1}^{M}\left(\left(\frac{\partial}{\partial \theta_{1}}\left(a_{k, 1}\right)^{*}\right)\right. \\
& \cdot\left(\frac{\partial}{\partial \phi_{2}} a_{k, 2}\right) a_{k^{\prime}, 1}\left(a_{k^{\prime}, 2}\right)^{*}+\left(\frac{\partial}{\partial \theta_{1}}\left(a_{k, 1}\right)^{*}\right) \\
& \cdot a_{k, 2} a_{k^{\prime}, 1}\left(\frac{\partial}{\partial \phi_{2}}\left(a_{k^{\prime}, 2}\right)^{*}\right)+\left(a_{k, 1}\right)^{*}\left(\frac{\partial}{\partial \phi_{2}} a_{k, 2}\right) \\
& \cdot\left(\frac{\partial}{\partial \theta_{1}} a_{k^{\prime}, 1}\right)\left(a_{k^{\prime}, 2}\right)^{*}+\left(a_{k, 1}\right)^{*} a_{k, 2}\left(\frac{\partial}{\partial \theta_{1}} a_{k^{\prime}, 1}\right)
\end{aligned}
$$




$$
\begin{aligned}
& \left.\cdot\left(\frac{\partial}{\partial \phi_{2}}\left(a_{k^{\prime}, 2}\right)^{*}\right)\right) \\
& \frac{\partial}{\partial \theta_{1}}\left(\frac{\partial}{\partial \theta_{2}} D(\Theta)\right)=\frac{\partial}{\partial \theta_{2}}\left(\frac{\partial}{\partial \theta_{1}} D(\Theta)\right), \\
& \frac{\partial^{2}}{\partial \theta_{2}^{2}} D(\Theta)=-\sum_{k=1}^{M} \sum_{k^{\prime}=1}^{M}\left(\left(a_{k, 1}\right)^{*}\left(\frac{\partial^{2}}{\partial \theta_{2}^{2}} a_{k, 2}\right)\right. \\
& \cdot a_{k^{\prime}, 1}\left(a_{k^{\prime}, 2}\right)^{*}+\left(a_{k, 1}\right)^{*}\left(\frac{\partial}{\partial \theta_{2}} a_{k, 2}\right) \\
& \cdot a_{k^{\prime}, 1}\left(\frac{\partial}{\partial \theta_{2}}\left(a_{k^{\prime}, 2}\right)^{*}\right)+\left(a_{k, 1}\right)^{*}\left(\frac{\partial}{\partial \theta_{2}} a_{k, 2}\right) \\
& \cdot a_{k^{\prime}, 1}\left(\frac{\partial}{\partial \theta_{2}}\left(a_{k^{\prime}, 2}\right)^{*}\right)+\left(a_{k, 1}\right)^{*} \\
& \left.\cdot a_{k, 2} a_{k^{\prime}, 1}\left(\frac{\partial^{2}}{\partial \theta_{2}^{2}}\left(a_{k^{\prime}, 2}\right)^{*}\right)\right) \\
& \frac{\partial}{\partial \phi_{1}}\left(\frac{\partial}{\partial \theta_{2}} D(\Theta)\right)=-\sum_{k=1}^{M} \sum_{k^{\prime}=1}^{M}\left(\left(\frac{\partial}{\partial \phi_{1}}\left(a_{k, 1}\right)^{*}\right)\right. \\
& \cdot\left(\frac{\partial}{\partial \theta_{2}} a_{k, 2}\right) a_{k^{\prime}, 1}\left(a_{k^{\prime}, 2}\right)^{*}+\left(a_{k, 1}\right)^{*}\left(\frac{\partial}{\partial \theta_{2}} a_{k, 2}\right) \\
& \cdot\left(\frac{\partial}{\partial \phi_{1}} a_{k^{\prime}, 1}\right)\left(a_{k^{\prime}, 2}\right)^{*}+\left(\frac{\partial}{\partial \phi_{1}}\left(a_{k, 1}\right)^{*}\right) \\
& \cdot a_{k, 2} a_{k^{\prime}, 1}\left(\frac{\partial}{\partial \theta_{2}}\left(a_{k^{\prime}, 2}\right)^{*}\right)+\left(a_{k, 1}\right)^{*} a_{k, 2}\left(\frac{\partial}{\partial \phi_{1}} a_{k^{\prime}, 1}\right) \\
& \left.\cdot\left(\frac{\partial}{\partial \theta_{2}}\left(a_{k^{\prime}, 2}\right)^{*}\right)\right) \\
& \frac{\partial}{\partial \phi_{2}}\left(\frac{\partial}{\partial \theta_{2}} D(\Theta)\right)=-\sum_{k=1}^{M} \sum_{k^{\prime}=1}^{M}\left(\left(a_{k, 1}\right)^{*}\right. \\
& \cdot\left(\frac{\partial}{\partial \phi_{2}}\left(\frac{\partial}{\partial \theta_{2}} a_{k, 2}\right)\right) a_{k^{\prime}, 1}\left(a_{k^{\prime}, 2}\right)^{*}+\left(a_{k, 1}\right)^{*} \\
& \cdot\left(\frac{\partial}{\partial \theta_{2}} a_{k, 2}\right) a_{k^{\prime}, 1}\left(\frac{\partial}{\partial \phi_{2}}\left(a_{k^{\prime}, 2}\right)^{*}\right)+\left(a_{k, 1}\right)^{*} \\
& \cdot\left(\frac{\partial}{\partial \phi_{2}} a_{k, 2}\right) a_{k^{\prime}, 1}\left(\frac{\partial}{\partial \theta_{2}}\left(a_{k^{\prime}, 2}\right)^{*}\right)+\left(a_{k, 1}\right)^{*} \\
& \left.\cdot a_{k, 2} a_{k^{\prime}, 1}\left(\frac{\partial}{\partial \phi_{2}}\left(\frac{\partial}{\partial \theta_{2}}\left(a_{k^{\prime}, 2}\right)^{*}\right)\right)\right) \\
& \frac{\partial}{\partial \theta_{1}}\left(\frac{\partial}{\partial \phi_{1}} D(\Theta)\right)=\frac{\partial}{\partial \phi_{1}}\left(\frac{\partial}{\partial \theta_{1}} D(\Theta)\right), \\
& \frac{\partial}{\partial \theta_{2}}\left(\frac{\partial}{\partial \phi_{2}} D(\Theta)\right)=\frac{\partial}{\partial \phi_{2}}\left(\frac{\partial}{\partial \theta_{2}} D(\Theta)\right),
\end{aligned}
$$

$$
\begin{aligned}
& \frac{\partial^{2}}{\partial \phi_{1}^{2}} D(\Theta)=-\sum_{k=1}^{M} \sum_{k^{\prime}=1}^{M}\left(\left(\frac{\partial^{2}}{\partial \phi_{1}^{2}}\left(a_{k, 1}\right)^{*}\right)\right. \\
& \cdot a_{k, 2} a_{k^{\prime}, 1}\left(a_{k^{\prime}, 2}\right)^{*}+\left(\frac{\partial}{\partial \phi_{1}}\left(a_{k, 1}\right)^{*}\right) a_{k, 2}\left(\frac{\partial}{\partial \phi_{1}} a_{k^{\prime}, 1}\right) \\
& \cdot\left(a_{k^{\prime}, 2}\right)^{*}+\left(\frac{\partial}{\partial \phi_{1}}\left(a_{k, 1}\right)^{*}\right) a_{k, 2}\left(\frac{\partial}{\partial \phi_{1}} a_{k^{\prime}, 1}\right)\left(a_{k^{\prime}, 2}\right)^{*} \\
& \left.+\left(a_{k, 1}\right)^{*} a_{k, 2}\left(\frac{\partial^{2}}{\partial \phi_{1}^{2}} a_{k^{\prime}, 1}\right)\left(a_{k^{\prime}, 2}\right)^{*}\right) \\
& \frac{\partial}{\partial \phi_{2}}\left(\frac{\partial}{\partial \phi_{1}} D(\Theta)\right)=-\sum_{k=1}^{M} \sum_{k^{\prime}=1}^{M}\left(\left(\frac{\partial}{\partial \phi_{1}}\left(a_{k, 1}\right)^{*}\right)\right. \\
& \cdot\left(\frac{\partial}{\partial \phi_{2}} a_{k, 2}\right) a_{k^{\prime}, 1}\left(a_{k^{\prime} .2}\right)^{*}+\left(\frac{\partial}{\partial \phi_{1}}\left(a_{k, 1}\right)^{*}\right) \\
& \cdot a_{k, 2} a_{k^{\prime}, 1}\left(\frac{\partial}{\partial \phi_{2}}\left(a_{k^{\prime}, 2}\right)^{*}\right)+\left(a_{k, 1}\right)^{*}\left(\frac{\partial}{\partial \phi_{2}} a_{k, 2}\right) \\
& \cdot\left(\frac{\partial}{\partial \phi_{1}} a_{k^{\prime}, 1}\right)\left(a_{k^{\prime}, 2}\right)^{*}+\left(a_{k, 1}\right)^{*} a_{k, 2}\left(\frac{\partial}{\partial \phi_{1}} a_{k^{\prime}, 1}\right) \\
& \left.\cdot\left(\frac{\partial}{\partial \phi_{2}}\left(a_{k^{\prime}, 2}\right)^{*}\right)\right) \\
& \frac{\partial}{\partial \theta_{1}}\left(\frac{\partial}{\partial \phi_{2}} D(\Theta)\right)=\frac{\partial}{\partial \phi_{2}}\left(\frac{\partial}{\partial \theta_{1}} D(\Theta)\right), \\
& \frac{\partial}{\partial \theta_{2}}\left(\frac{\partial}{\partial \phi_{2}} D(\Theta)\right)=\frac{\partial}{\partial \phi_{2}}\left(\frac{\partial}{\partial \theta_{2}} D(\Theta)\right), \\
& \frac{\partial}{\partial \phi_{1}}\left(\frac{\partial}{\partial \phi_{2}} D(\Theta)\right)=\frac{\partial}{\partial \phi_{2}}\left(\frac{\partial}{\partial \phi_{1}} D(\Theta)\right), \\
& \frac{\partial^{2}}{\partial \phi_{2}^{2}} D(\Theta)=\sum_{k=1}^{M} \sum_{k^{\prime}=1}^{M}\left(\left(a_{k, 1}\right)^{*}\left(\frac{\partial^{2}}{\partial \phi_{2}^{2}} a_{k, 2}\right) a_{k^{\prime}, 1}\left(a_{k^{\prime}, 2}\right)^{*}\right. \\
& +\left(a_{k, 1}\right)^{*}\left(\frac{\partial}{\partial \phi_{2}} a_{k, 2}\right) a_{k^{\prime}, 1}\left(\frac{\partial}{\partial \phi_{2}}\left(a_{k^{\prime}, 2}\right)^{*}\right)+\left(a_{k, 1}\right)^{*} \\
& \cdot\left(\frac{\partial}{\partial \phi_{2}} a_{k, 2}\right) a_{k^{\prime}, 1}\left(\frac{\partial}{\partial \phi_{2}}\left(a_{k^{\prime}, 2}\right)^{*}\right)+\left(a_{k, 1}\right)^{*} \\
& \left.\cdot a_{k, 2} a_{k^{\prime}, 1}\left(\frac{\partial^{2}}{\partial \phi_{2}^{2}}\left(a_{k^{\prime}, 2}\right)^{*}\right)\right) \text {. }
\end{aligned}
$$

\section{G. The First-Order Derivatives of $P_{k l, \theta_{1}}(\Theta)$, $P_{k l, \theta_{2}}(\Theta), P_{k l, \phi_{1}}(\Theta)$, and $P_{k l, \phi_{2}}(\Theta)$}

The first partial derivatives of $P_{k l, \theta_{1}}(\Theta), P_{k l, \theta_{2}}(\Theta), P_{k l, \phi_{1}}(\Theta)$, and $P_{k l, \phi_{2}}(\Theta)$ in (27) with respect to each argument can be expressed as

$$
\frac{\partial}{\partial \theta_{i}} P_{k l, \theta_{1}}(\Theta)=\frac{\left(\left(\left(\partial / \partial \theta_{i}\right)\left(\partial / \partial \theta_{1}\right) S_{k l}(\Theta)\right) D(\Theta)+\left(\left(\partial / \partial \theta_{1}\right) S_{k l}(\Theta)\right)\left(\left(\partial / \partial \theta_{i}\right) D(\Theta)\right)\right)(D(\Theta))^{2}}{(D(\Theta))^{4}}
$$




$$
\begin{aligned}
& -\frac{\left(\left(\left(\partial / \partial \theta_{i}\right) S_{k l}(\Theta)\right)\left(\left(\partial / \partial \theta_{1}\right) D(\Theta)\right)+S_{k l}(\Theta)\left(\left(\partial / \partial \theta_{i}\right)\left(\partial / \partial \theta_{1}\right) D(\Theta)\right)\right)(D(\Theta))^{2}}{(D(\Theta))^{4}} \\
& -\frac{\left(\left(\left(\partial / \partial \theta_{1}\right) S_{k l}(\Theta)\right) D(\Theta)-S_{k l}(\Theta)\left(\left(\partial / \partial \theta_{1}\right) D(\Theta)\right)\right)\left(\left(\partial / \partial \theta_{i}\right) D(\Theta)\right)^{2}}{(D(\Theta))^{4}} \quad i=1,2, \\
& \frac{\partial}{\partial \phi_{i}} P_{k l, \theta_{1}}(\Theta)=\frac{\left(\left(\left(\partial / \partial \phi_{i}\right)\left(\partial / \partial \theta_{1}\right) S_{k l}(\Theta)\right) D(\Theta)+\left(\left(\partial / \partial \theta_{1}\right) S_{k l}(\Theta)\right)\left(\left(\partial / \partial \phi_{i}\right) D(\Theta)\right)\right)(D(\Theta))^{2}}{(D(\Theta))^{4}} \\
& -\frac{\left(\left(\left(\partial / \partial \phi_{i}\right) S_{k l}(\Theta)\right)\left(\left(\partial / \partial \theta_{1}\right) D(\Theta)\right)+S_{k l}(\Theta)\left(\left(\partial / \partial \phi_{i}\right)\left(\partial / \partial \theta_{1}\right) D(\Theta)\right)\right)(D(\Theta))^{2}}{(D(\Theta))^{4}} \\
& -\frac{\left(\left(\left(\partial / \partial \theta_{1}\right) S_{k l}(\Theta)\right) D(\Theta)-S_{k l}(\Theta)\left(\left(\partial / \partial \theta_{1}\right) D(\Theta)\right)\right)\left(\left(\partial / \partial \phi_{i}\right) D(\Theta)\right)^{2}}{(D(\Theta))^{4}} \quad i=1,2, \\
& \frac{\partial}{\partial \theta_{1}} P_{k l, \theta_{2}}(\Theta)=\frac{\partial}{\partial \theta_{1}}\left(\frac{\partial}{\partial \theta_{2}} P_{k l}(\Theta)\right)=\frac{\partial}{\partial \theta_{2}}\left(\frac{\partial}{\partial \theta_{1}} P_{k l}(\Theta)\right)=\frac{\partial}{\partial \theta_{2}} P_{k l, \theta_{1}}(\Theta), \\
& \frac{\partial}{\partial \phi_{i}} P_{k l, \theta_{2}}(\Theta)=\frac{\left(\left(\left(\partial / \partial \phi_{i}\right)\left(\partial / \partial \theta_{2}\right) S_{k l}(\Theta)\right) D(\Theta)+\left(\left(\partial / \partial \theta_{2}\right) S_{k l}(\Theta)\right)\left(\left(\partial / \partial \phi_{i}\right) D(\Theta)\right)\right)(D(\Theta))^{2}}{(D(\Theta))^{4}} \\
& -\frac{\left(\left(\left(\partial / \partial \phi_{i}\right) S_{k l}(\Theta)\right)\left(\left(\partial / \partial \theta_{2}\right) D(\Theta)\right)+S_{k l}(\Theta)\left(\left(\partial / \partial \phi_{i}\right)\left(\partial / \partial \theta_{2}\right) D(\Theta)\right)\right)(D(\Theta))^{2}}{(D(\Theta))^{4}} \\
& -\frac{\left(\left(\left(\partial / \partial \theta_{2}\right) S_{k l}(\Theta)\right) D(\Theta)-S_{k l}(\Theta)\left(\left(\partial / \partial \theta_{2}\right) D(\Theta)\right)\right)\left(\left(\partial / \partial \phi_{i}\right) D(\Theta)\right)^{2}}{(D(\Theta))^{4}} \quad i=1,2, \\
& \frac{\partial}{\partial \theta_{1}} P_{k l, \phi_{1}}(\Theta)=\frac{\partial}{\partial \theta_{1}}\left(\frac{\partial}{\partial \phi_{1}} P_{k l}(\Theta)\right)=\frac{\partial}{\partial \phi_{1}}\left(\frac{\partial}{\partial \theta_{1}} P_{k l}(\Theta)\right)=\frac{\partial}{\partial \phi_{1}} P_{k l, \theta_{1}}(\Theta), \\
& \frac{\partial}{\partial \theta_{2}} P_{k l, \phi_{1}}(\Theta)=\frac{\partial}{\partial \theta_{2}}\left(\frac{\partial}{\partial \phi_{1}} P_{k l}(\Theta)\right)=\frac{\partial}{\partial \phi_{1}}\left(\frac{\partial}{\partial \theta_{2}} P_{k l}(\Theta)\right)=\frac{\partial}{\partial \phi_{1}} P_{k l, \theta_{2}}(\Theta), \\
& \frac{\partial}{\partial \phi_{i}} P_{k l, \phi_{1}}(\Theta)=\frac{\left(\left(\left(\partial / \partial \phi_{i}\right)\left(\partial / \partial \phi_{1}\right) S_{k l}(\Theta)\right) D(\Theta)+\left(\left(\partial / \partial \phi_{1}\right) S_{k l}(\Theta)\right)\left(\left(\partial / \partial \phi_{i}\right) D(\Theta)\right)\right)(D(\Theta))^{2}}{(D(\Theta))^{4}} \\
& -\frac{\left(\left(\left(\partial / \partial \phi_{i}\right) S_{k l}(\Theta)\right)\left(\left(\partial / \partial \phi_{1}\right) D(\Theta)\right)+S_{k l}(\Theta)\left(\left(\partial / \partial \phi_{i}\right)\left(\partial / \partial \phi_{1}\right) D(\Theta)\right)\right)(D(\Theta))^{2}}{(D(\Theta))^{4}} \\
& -\frac{\left(\left(\left(\partial / \partial \phi_{1}\right) S_{k l}(\Theta)\right) D(\Theta)-S_{k l}(\Theta)\left(\left(\partial / \partial \phi_{1}\right) D(\Theta)\right)\right)\left(\left(\partial / \partial \phi_{i}\right) D(\Theta)\right)^{2}}{(D(\Theta))^{4}} \quad i=1,2, \\
& \frac{\partial}{\partial \theta_{1}} P_{k l, \phi_{2}}(\Theta)=\frac{\partial}{\partial \theta_{1}}\left(\frac{\partial}{\partial \phi_{2}} P_{k l}(\Theta)\right)=\frac{\partial}{\partial \phi_{2}}\left(\frac{\partial}{\partial \theta_{1}} P_{k l}(\Theta)\right)=\frac{\partial}{\partial \phi_{2}} P_{k l, \theta_{1}}(\Theta), \\
& \frac{\partial}{\partial \theta_{2}} P_{k l, \phi_{2}}(\Theta)=\frac{\partial}{\partial \theta_{2}}\left(\frac{\partial}{\partial \phi_{2}} P_{k l}(\Theta)\right)=\frac{\partial}{\partial \phi_{2}}\left(\frac{\partial}{\partial \theta_{2}} P_{k l}(\Theta)\right)=\frac{\partial}{\partial \phi_{2}} P_{k l, \theta_{2}}(\Theta), \\
& \frac{\partial}{\partial \phi_{1}} P_{k l, \phi_{2}}(\Theta)=\frac{\partial}{\partial \phi_{1}}\left(\frac{\partial}{\partial \phi_{2}} P_{k l}(\Theta)\right)=\frac{\partial}{\partial \phi_{2}}\left(\frac{\partial}{\partial \phi_{1}} P_{k l}(\Theta)\right)=\frac{\partial}{\partial \phi_{2}} P_{k l, \phi_{1}}(\Theta) .
\end{aligned}
$$




\section{H. The First-Order Derivatives of $(D(\Theta))^{2}$}

For use in Appendix G, the first partial derivatives of $(D(\Theta))^{2}$ in (28) with respect to each argument are expressed as

$$
\begin{aligned}
\frac{\partial}{\partial \theta_{i}}(D(\Theta))^{2}= & -2 M^{2} \frac{\partial}{\partial \theta_{i}}|Q(\Theta)|^{2} \\
& +\left(\frac{\partial}{\partial \theta_{i}}|Q(\Theta)|^{4}\right) \quad i=1,2, \\
\frac{\partial}{\partial \phi_{i}}(D(\Theta))^{2}= & -2 M^{2} \frac{\partial}{\partial \phi_{i}}|Q(\Theta)|^{2} \\
& +\left(\frac{\partial}{\partial \phi_{i}}|Q(\Theta)|^{4}\right) \quad i=1,2 .
\end{aligned}
$$

\section{The First-Order Derivatives of $|Q(\Theta)|^{2}$}

For use in Appendix $\mathrm{H}$, the first-order partial derivatives of $|Q(\Theta)|^{2}$ in (21) with respect to each argument are expressed as

$$
\begin{aligned}
& \frac{\partial}{\partial \theta_{i}}(Q(\Theta))^{2} \\
& =\sum_{k=1}^{M} \sum_{k^{\prime}=1}^{M}\left(\left(\frac{\partial}{\partial \theta_{i}}\left(a_{k, 1}\right)^{*}\right) a_{k, 2} 2 a_{k^{\prime}, 1}\left(a_{k^{\prime}, 2}\right)^{*}\right. \\
& \left.\quad+\left(a_{k, 1}\right)^{*} a_{k, 2}\left(\frac{\partial}{\partial \theta_{i}} a_{k^{\prime}, 1}\right)\left(a_{k^{\prime}, 2}\right)^{*}\right) \quad i=1,2, \\
& \frac{\partial}{\partial \phi_{i}}(Q(\Theta))^{2} \\
& \quad=\sum_{k=1}^{M} \sum_{k^{\prime}=1}^{M}\left(\left(\frac{\partial}{\partial \phi_{i}}\left(a_{k, 1}\right)^{*}\right) a_{k, 2} 2 a_{k^{\prime}, 1}\left(a_{k^{\prime}, 2}\right)^{*}\right. \\
& \left.\quad+\left(a_{k, 1}\right)^{*} a_{k, 2}\left(\frac{\partial}{\partial \phi_{i}} a_{k^{\prime}, 1}\right)\left(a_{k^{\prime}, 2}\right)^{*}\right) \quad i=1,2 .
\end{aligned}
$$

\section{J. The First-Order Derivatives of $|Q(\Theta)|^{4}$}

For use in Appendix $\mathrm{H}$, the first-order partial derivatives of $|Q(\Theta)|^{4}$ in (29) with respect to each argument are expressed as

$$
\begin{aligned}
& \frac{\partial}{\partial \theta_{i}}(Q(\Theta))^{4} \\
& =\sum_{k=1}^{M} \sum_{k^{\prime}=1}^{M} \sum_{q=1}^{M} \sum_{q^{\prime}=1}^{M}\left(\left(\frac{\partial}{\partial \theta_{i}}\left(a_{k, 1}\right)^{*}\right) a_{k, 2} a_{k^{\prime}, 1}\left(a_{k, 2}\right)^{*}\left(a_{q, 1}\right)^{*} a_{q, 2} a_{q^{\prime}, 1}\left(a_{q^{\prime}, 2}\right)^{*}\right. \\
& +\left(a_{k, 1}\right)^{*} a_{k, 2}\left(\frac{\partial}{\partial \theta_{i}} a_{k^{\prime}, 1}\right)\left(a_{k, 2}\right)^{*}\left(a_{q, 1}\right)^{*} a_{q, 2} a_{q^{\prime}, 1}\left(a_{q^{\prime}, 2}\right)^{*} \\
& +\left(a_{k, 1}\right)^{*} a_{k, 2} a_{k^{\prime}, 1}\left(a_{k, 2}\right)^{*}\left(\frac{\partial}{\partial \theta_{i}}\left(a_{q, 1}\right)^{*}\right) a_{q, 2} a_{q^{\prime}, 1}\left(a_{q^{\prime}, 2}\right)^{*} \\
& \left.+\left(a_{k, 1}\right)^{*} a_{k, 2} a_{k^{\prime}, 1}\left(a_{k, 2}\right)^{*}\left(a_{q, 1}\right)^{*} a_{q, 2}\left(\frac{\partial}{\partial \theta_{i}}\left(a_{q, 1}\right)\right)\left(a_{q^{\prime}, 2}\right)^{*}\right) \quad i=1,2,
\end{aligned}
$$

$$
\frac{\partial}{\partial \phi_{i}}(Q(\Theta))^{4}
$$

$$
\begin{aligned}
& =\sum_{k=1}^{M} \sum_{k^{\prime}=1}^{M} \sum_{q=1}^{M} \sum_{q^{\prime}=1}^{M}\left(\left(\frac{\partial}{\partial \phi_{i}}\left(a_{k, 1}\right)^{*}\right) a_{k, 2} a_{k^{\prime}, 1}\left(a_{k, 2}\right)^{*}\left(a_{q, 1}\right)^{*} a_{q, 2} a_{q^{\prime}, 1}\left(a_{q^{\prime}, 2}\right)^{*}\right. \\
& +\left(a_{k, 1}\right)^{*} a_{k, 2}\left(\frac{\partial}{\partial \phi_{i}} a_{k^{\prime}, 1}\right)\left(a_{k, 2}\right)^{*}\left(a_{q, 1}\right)^{*} a_{q, 2} a_{q^{\prime}, 1}\left(a_{q^{\prime}, 2}\right)^{*} \\
& +\left(a_{k, 1}\right)^{*} a_{k, 2} a_{k^{\prime}, 1}\left(a_{k, 2}\right)^{*}\left(\frac{\partial}{\partial \phi_{i}}\left(a_{q, 1}\right)^{*}\right) a_{q, 2} a_{q^{\prime}, 1}\left(a_{q^{\prime}, 2}\right)^{*} \\
& \left.+\left(a_{k, 1}\right)^{*} a_{k, 2} a_{k^{\prime}, 1}\left(a_{k, 2}\right)^{*}\left(a_{q, 1}\right)^{*} a_{q, 2}\left(\frac{\partial}{\partial \phi_{i}}\left(a_{q, 1}\right)\right)\left(a_{q^{\prime}, 2}\right)^{*}\right) \quad i=1,2 .
\end{aligned}
$$

\section{K. The Second-Oder Derivatives of $a_{m}\left(\theta_{i}, \phi_{i}\right)$}

For use in Appendices E, F, and L, the second partial derivatives of $a_{m}\left(\theta_{i}, \phi_{i}\right)$ in (18) with respect to each argument are expressed as

$$
\begin{gathered}
\frac{\partial^{2}}{\partial \theta_{i}^{2}} a_{m}\left(\theta_{i}, \phi_{i}\right)=-j \frac{2 \pi r}{\lambda} \\
\cdot \cos \phi_{i}\left(\cos \left(\theta_{i}-\frac{2 \pi}{M}(m-1)\right) a_{m}\left(\theta_{i}, \phi_{i}\right)\right. \\
\left.-\sin \left(\theta_{i}-\frac{2 \pi}{M}(m-1)\right) \frac{\partial}{\partial \theta_{i}} a_{m}\left(\theta_{i}, \phi_{i}\right)\right), \\
\frac{\partial^{2}}{\partial \phi_{i}^{2}} a_{m}\left(\theta_{i}, \phi_{i}\right)=-j \frac{2 \pi r}{\lambda} \cos \left(\theta_{i}-\frac{2 \pi}{M}(m-1)\right) \\
\cdot\left(\cos \phi_{i} a_{m}\left(\theta_{i}, \phi_{i}\right)-\sin \phi_{i} \frac{\partial}{\partial \phi_{i}} a_{m}\left(\theta_{i}, \phi_{i}\right)\right), \\
\frac{\partial}{\partial \phi_{i}} \frac{\partial}{\partial \theta_{i}} a_{m}\left(\theta_{i}, \phi_{i}\right)=j \frac{2 \pi r}{\lambda} \sin \left(\theta_{i}-\frac{2 \pi}{M}(m-1)\right) \\
\quad\left(\sin \phi_{i} a_{m}\left(\theta_{i}, \phi_{i}\right)-\cos \phi_{i} \frac{\partial}{\partial \phi_{i}} a_{m}\left(\theta_{i}, \phi_{i}\right)\right) .
\end{gathered}
$$

\section{The Second-Order Derivatives of $Q(\Theta)$}

For use in Appendix F, the second partial derivatives of $Q(\Theta)$ in (17) with respect to each argument are expressed as

$$
\begin{array}{r}
\frac{\partial}{\partial \theta_{i}} \frac{\partial}{\partial \theta_{1}} Q(\Theta)=\sum_{k=1}^{M}\left(\left(\frac{\partial}{\partial \theta_{1}}\left(a_{k, 1}\right)^{*}\right)\left(\frac{\partial}{\partial \theta_{i}} a_{k, 2}\right)\right) \\
i=1,2, \\
\frac{\partial}{\partial \phi_{i}} \frac{\partial}{\partial \theta_{1}} Q(\Theta)=\sum_{k=1}^{M}\left(\left(\frac{\partial}{\partial \phi_{1}}\left(a_{k, 1}\right)^{*}\right)\left(\frac{\partial}{\partial \theta_{i}} a_{k, 2}\right)\right) \\
i=1,2,
\end{array}
$$




$$
\begin{aligned}
& \frac{\partial}{\partial \theta_{1}}\left(\frac{\partial}{\partial \theta_{2}} Q(\Theta)\right)=\frac{\partial}{\partial \theta_{2}}\left(\frac{\partial}{\partial \theta_{1}} Q(\Theta)\right), \\
& \frac{\partial^{2}}{\partial \theta_{2}^{2}} Q(\Theta)=\sum_{k=1}^{M}\left(\left(a_{k, 1}\right)^{*}\left(\frac{\partial^{2}}{\partial \theta_{2}^{2}} a_{k, 2}\right)\right), \\
& \frac{\partial}{\partial \phi_{i}} \frac{\partial}{\partial \theta_{1}} Q(\Theta)=\sum_{k=1}^{M}\left(\left(\frac{\partial}{\partial \phi_{i}}\left(a_{k, 1}\right)^{*}\right)\left(\frac{\partial}{\partial \theta_{2}} a_{k, 2}\right)\right) \\
& \frac{\partial}{\partial \theta_{i}}\left(\frac{\partial}{\partial \phi_{1}} Q(\Theta)\right)=\frac{\partial}{\partial \phi_{1}}\left(\frac{\partial}{\partial \theta_{i}} Q(\Theta)\right) \quad i=1,2, \\
& \frac{\partial}{\partial \phi_{i}}\left(\frac{\partial}{\partial \phi_{1}} Q(\Theta)\right)=\sum_{k=1}^{M}\left(\left(\frac{\partial}{\partial \phi_{1}}\left(a_{k, 1}\right)^{*}\right)\left(\frac{\partial}{\partial \phi_{i}} a_{k, 2}\right)\right) \\
& \frac{\partial}{\partial \theta_{i}}\left(\frac{\partial}{\partial \phi_{2}} Q(\Theta)\right)=\frac{\partial}{\partial \phi_{2}}\left(\frac{\partial}{\partial \theta_{i}} Q(\Theta)\right) \quad i=1,2, \\
& i=1,2,
\end{aligned}
$$

$$
\begin{aligned}
\frac{\partial}{\partial \phi_{1}}\left(\frac{\partial}{\partial \phi_{2}} Q(\Theta)\right) & =\frac{\partial}{\partial \phi_{2}}\left(\frac{\partial}{\partial \phi_{1}} Q(\Theta)\right), \\
\frac{\partial^{2}}{\partial \phi_{2}^{2}} Q(\Theta) & =\sum_{k=1}^{M}\left(\left(a_{k, 1}\right)^{*}\left(\frac{\partial^{2}}{\partial \phi_{2}^{2}} a_{k, 2}\right)\right) .
\end{aligned}
$$

\section{Second-Order Central Moment of Zero-Mean Complex Gaussian Random Variable with Variance $\sigma^{2}$}

Depending on how $a, b, d$, and $e$ are related, we define four cases.

Case 1. $a=b$ and $d=e$.

Case 2. $a \neq b$ and $d=e$.

Case 3. $a=b$ and $d \neq e$.

Case 4. $a \neq b$ and $d \neq e$.

(i) $E\left[n_{a}\left(t_{d}\right) n_{b}^{*}\left(t_{e}\right)\right]$ :

$$
\begin{aligned}
E & {\left[n_{a}\left(t_{d}\right) n_{b}^{*}\left(t_{e}\right)\right]=E\left[\left(\operatorname{Re}\left[n_{a}\left(t_{d}\right)\right]+j \operatorname{Im}\left[n_{a}\left(t_{d}\right)\right]\right)\left(\operatorname{Re}\left[n_{b}\left(t_{e}\right)\right]-j \operatorname{Im}\left[n_{b}\left(t_{e}\right)\right]\right)\right] } \\
& =E\left[\operatorname{Re}\left[n_{a}\left(t_{d}\right)\right] \operatorname{Re}\left[n_{b}\left(t_{e}\right)\right]-j \operatorname{Re}\left[n_{a}\left(t_{d}\right)\right] \operatorname{Im}\left[n_{b}\left(t_{e}\right)\right]+j \operatorname{Im}\left[n_{a}\left(t_{d}\right)\right] \operatorname{Re}\left[n_{b}\left(t_{e}\right)\right]+\operatorname{Im}\left[n_{a}\left(t_{d}\right)\right] \operatorname{Im}\left[n_{b}\left(t_{e}\right)\right]\right] .
\end{aligned}
$$

For Case $1, E\left[n_{a}\left(t_{d}\right) n_{b}^{*}\left(t_{e}\right)\right]$ is given by

$$
\begin{aligned}
E\left[n_{a}\left(t_{d}\right) n_{b}^{*}\left(t_{e}\right)\right]=E\left[n_{a}\left(t_{d}\right) n_{a}^{*}\left(t_{d}\right)\right] \\
=E\left[\operatorname{Re}\left[n_{a}\left(t_{d}\right)\right] \operatorname{Re}\left[n_{a}\left(t_{d}\right)\right]\right] \\
\quad+E\left[\operatorname{Im}\left[n_{a}\left(t_{d}\right)\right] \operatorname{Im}\left[n_{a}\left(t_{d}\right)\right]\right] \\
\quad-j E\left[\operatorname{Re}\left[n_{a}\left(t_{d}\right)\right]\right] E\left[\operatorname{Im}\left[n_{a}\left(t_{d}\right)\right]\right] \\
\quad+j E\left[\operatorname{Im}\left[n_{a}\left(t_{d}\right)\right]\right] E\left[\operatorname{Re}\left[n_{a}\left(t_{d}\right)\right]\right] \\
=\frac{\sigma^{2}}{2}+\frac{\sigma^{2}}{2}-0+0=\sigma^{2} .
\end{aligned}
$$

Similarly, it can be shown that $E\left[n_{a}\left(t_{d}\right) n_{b}^{*}\left(t_{e}\right)\right]$ is identically zero for Cases 2-4:

$$
E\left[n_{a}\left(t_{d}\right) n_{b}^{*}\left(t_{e}\right)\right]=0 \quad \text { for Cases } 2-4 \text {. }
$$

Note that, in deriving (M.1)-(M.3), we used the fact that the real part and the imaginary part of noise are independent and identically distributed with $N(0$, $\left.\sigma^{2} / 2\right)$.

(ii) $E\left[n_{a}^{*}\left(t_{d}\right) n_{b}^{*}\left(t_{e}\right)\right]$ :

Using the same algebraic manipulation used in evaluating $E\left[n_{a}\left(t_{d}\right) n_{b}^{*}\left(t_{e}\right)\right]$, it can be shown that $E\left[n_{a}^{*}\left(t_{d}\right) n_{b}^{*}\left(t_{e}\right)\right]$ is equal to zero for Cases $1-4$ :

$E\left[n_{a}^{*}\left(t_{d}\right) n_{b}^{*}\left(t_{e}\right)\right]=0$ for Cases $1-4$. (iii) $E\left[n_{a}\left(t_{d}\right) n_{b}\left(t_{e}\right)\right]$ :

Using the same algebraic manipulation used in evaluating $E\left[n_{a}\left(t_{d}\right) n_{b}^{*}\left(t_{e}\right)\right]$, it can be shown that $E\left[n_{a}\left(t_{d}\right) n_{b}\left(t_{e}\right)\right]$ is equal to zero for Cases 1-4:

$$
E\left[n_{a}\left(t_{d}\right) n_{b}\left(t_{e}\right)\right]=0 \text { for Cases } 1-4 \text {. }
$$

\section{N. Third-Order Central Moment of Zero-Mean Complex Gaussian Random Variable with Variance $\sigma^{2}$}

We define ten cases depending on how $a, b, c, d$, and $e$ are related.

Case 1. $a=b$ and $b=c$ and $e=d$.

Case 2. $a=b$ and $b \neq c$ and $e=d$.

Case 3. $a \neq b$ and $b=c$ and $e=d$.

Case 4. $a=c$ and $c \neq b$ and $e=d$.

Case 5. $a \neq b$ and $b \neq c$ and $e=d$.

Case 6. $a=b$ and $b=c$ and $e \neq d$.

Case 7. $a=b$ and $b \neq c$ and $e \neq d$. 
Case 8. $a \neq b$ and $b=c$ and $e \neq d$.

Case 9. $a=c$ and $c \neq b$ and $e \neq d$.

Case 10. $a \neq b$ and $b \neq c$ and $e \neq d$.

(i) $E\left[n_{a}\left(t_{d}\right) n_{b}^{*}\left(t_{e}\right) n_{c}\left(t_{e}\right)\right]:$

$$
\begin{aligned}
E[ & \left.n_{a}\left(t_{d}\right) n_{b}^{*}\left(t_{e}\right) n_{c}\left(t_{e}\right)\right] \\
& =E\left[\left(\operatorname{Re}\left[n_{a}\left(t_{d}\right)\right]+j \operatorname{Im}\left[n_{a}\left(t_{d}\right)\right]\right)\right. \\
& \cdot\left(\operatorname{Re}\left[n_{b}\left(t_{e}\right)\right]-j \operatorname{Im}\left[n_{b}\left(t_{e}\right)\right]\right) \\
& \left.\cdot\left(\operatorname{Re}\left[n_{c}\left(t_{e}\right)\right]+j \operatorname{Im}\left[n_{c}\left(t_{e}\right)\right]\right)\right]=E\left[\operatorname{Re}\left[n_{a}\left(t_{d}\right)\right]\right. \\
& \left.\cdot \operatorname{Re}\left[n_{b}\left(t_{e}\right)\right] \operatorname{Re}\left[n_{c}\left(t_{e}\right)\right]\right]+j E\left[\operatorname{Re}\left[n_{a}\left(t_{d}\right)\right]\right. \\
& \left.\cdot \operatorname{Re}\left[n_{b}\left(t_{e}\right)\right] \operatorname{Im}\left[n_{c}\left(t_{e}\right)\right]\right]-j E\left[\operatorname{Re}\left[n_{a}\left(t_{d}\right)\right]\right. \\
& \left.\cdot \operatorname{Im}\left[n_{b}\left(t_{e}\right)\right] \operatorname{Re}\left[n_{c}\left(t_{e}\right)\right]\right]+j E\left[\operatorname{Im}\left[n_{a}\left(t_{d}\right)\right]\right. \\
& \left.\cdot \operatorname{Re}\left[n_{b}\left(t_{e}\right)\right] \operatorname{Re}\left[n_{c}\left(t_{e}\right)\right]\right]+E\left[\operatorname{Re}\left[n_{a}\left(t_{d}\right)\right]\right. \\
& \left.\cdot \operatorname{Im}\left[n_{b}\left(t_{e}\right)\right] \operatorname{Im}\left[n_{c}\left(t_{e}\right)\right]\right]-E\left[\operatorname{Im}\left[n_{a}\left(t_{d}\right)\right]\right. \\
& \left.\cdot \operatorname{Re}\left[n_{b}\left(t_{e}\right)\right] \operatorname{Im}\left[n_{c}\left(t_{e}\right)\right]\right]+E\left[\operatorname{Im}\left[n_{a}\left(t_{d}\right)\right]\right. \\
& \left.\cdot \operatorname{Im}\left[n_{b}\left(t_{e}\right)\right] \operatorname{Re}\left[n_{c}\left(t_{e}\right)\right]\right]+j E\left[\operatorname{Im}\left[n_{a}\left(t_{d}\right)\right]\right. \\
& \left.\cdot \operatorname{Im}\left[n_{b}\left(t_{e}\right)\right] \operatorname{Im}\left[n_{c}\left(t_{e}\right)\right]\right] .
\end{aligned}
$$$$
E\left[n_{a}\left(t_{d}\right) n_{b}^{*}\left(t_{e}\right) n_{c}\left(t_{e}\right)\right] \text { for Case } 1 \text { is identically zero: }
$$$$
E\left[n_{a}\left(t_{d}\right) n_{b}^{*}\left(t_{e}\right) n_{c}\left(t_{e}\right)\right]
$$$$
=E\left[n_{a}\left(t_{d}\right) n_{a}^{*}\left(t_{d}\right) n_{a}\left(t_{d}\right]\right)
$$$$
=E\left[\operatorname{Re}\left[n_{a}\left(t_{d}\right)\right] \operatorname{Re}\left[n_{a}\left(t_{d}\right)\right] \operatorname{Re}\left[n_{a}\left(t_{d}\right)\right]\right]
$$$$
+j E\left[\operatorname{Re}\left[n_{a}\left(t_{d}\right)\right] \operatorname{Re}\left[n_{a}\left(t_{d}\right)\right]\right] E\left[\operatorname{Im}\left[n_{a}\left(t_{d}\right)\right]\right]
$$$$
-j E\left[\operatorname{Re}\left[n_{a}\left(t_{d}\right)\right] \operatorname{Re}\left[n_{a}\left(t_{d}\right)\right]\right] E\left[\operatorname{Im}\left[n_{a}\left(t_{d}\right)\right]\right]
$$$$
+j E\left[\operatorname{Im}\left[n_{a}\left(t_{d}\right)\right]\right] E\left[\operatorname{Re}\left[n_{a}\left(t_{d}\right)\right] \operatorname{Re}\left[n_{a}\left(t_{d}\right)\right]\right]
$$$$
+E\left[\operatorname{Re}\left[n_{a}\left(t_{d}\right)\right]\right] E\left[\operatorname{Im}\left[n_{a}\left(t_{d}\right)\right] \operatorname{Im}\left[n_{a}\left(t_{d}\right)\right]\right]
$$$$
-E\left[\operatorname{Im}\left[n_{a}\left(t_{d}\right)\right] \operatorname{Im}\left[n_{a}\left(t_{d}\right)\right]\right] E\left[\operatorname{Re}\left[n_{a}\left(t_{d}\right)\right]\right]
$$$$
+E\left[\operatorname{Im}\left[n_{a}\left(t_{d}\right)\right] \operatorname{Im}\left[n_{a}\left(t_{d}\right)\right]\right] E\left[\operatorname{Re}\left[n_{a}\left(t_{d}\right)\right]\right]
$$$$
+j E\left[\operatorname{Im}\left[n_{a}\left(t_{d}\right)\right] \operatorname{Im}\left[n_{a}\left(t_{d}\right)\right] \operatorname{Im}\left[n_{a}\left(t_{d}\right)\right]\right]
$$$$
=0+j\left(\frac{\sigma^{2}}{2}\right) \times 0-j\left(\frac{\sigma^{2}}{2}\right) \times 0+j\left(\frac{\sigma^{2}}{2}\right) \times 0
$$$$
+\left(\frac{\sigma^{2}}{2}\right) \times 0-\left(\frac{\sigma^{2}}{2}\right) \times 0+\left(\frac{\sigma^{2}}{2}\right) \times 0+j \times 0
$$$$
=0 \text {. }
$$

(ii) $E\left[n_{a}^{*}\left(t_{d}\right) n_{b}^{*}\left(t_{e}\right) n_{c}\left(t_{e}\right)\right]$ :

$$
\begin{aligned}
E[ & \left.n_{a}^{*}\left(t_{d}\right) n_{b}^{*}\left(t_{e}\right) n_{c}\left(t_{e}\right)\right] \\
& =E\left[\left(\operatorname{Re}\left[n_{a}\left(t_{d}\right)\right]-j \operatorname{Im}\left[n_{a}\left(t_{d}\right)\right]\right)\right. \\
& \cdot\left(\operatorname{Re}\left[n_{b}\left(t_{e}\right)\right]-j \operatorname{Im}\left[n_{b}\left(t_{e}\right)\right]\right) \\
& \left.\cdot\left(\operatorname{Re}\left[n_{c}\left(t_{e}\right)\right]+j \operatorname{Im}\left[n_{c}\left(t_{e}\right)\right]\right)\right]=E\left[\operatorname{Re}\left[n_{a}\left(t_{d}\right)\right]\right. \\
& \left.\cdot \operatorname{Re}\left[n_{b}\left(t_{e}\right)\right] \operatorname{Re}\left[n_{c}\left(t_{e}\right)\right]\right]+j E\left[\operatorname{Re}\left[n_{a}\left(t_{d}\right)\right]\right. \\
& \left.\cdot \operatorname{Re}\left[n_{b}\left(t_{e}\right)\right] \operatorname{Im}\left[n_{c}\left(t_{e}\right)\right]\right]-j E\left[\operatorname{Re}\left[n_{a}\left(t_{d}\right)\right]\right. \\
& \left.\cdot \operatorname{Im}\left[n_{b}\left(t_{e}\right)\right] \operatorname{Re}\left[n_{c}\left(t_{e}\right)\right]\right]-j E\left[\operatorname{Im}\left[n_{a}\left(t_{d}\right)\right]\right. \\
& \left.\cdot \operatorname{Re}\left[n_{b}\left(t_{e}\right)\right] \operatorname{Re}\left[n_{c}\left(t_{e}\right)\right]\right]+E\left[\operatorname{Re}\left[n_{a}\left(t_{d}\right)\right]\right. \\
& \left.\cdot \operatorname{Im}\left[n_{b}\left(t_{e}\right)\right] \operatorname{Im}\left[n_{c}\left(t_{e}\right)\right]\right]+E\left[\operatorname{Im}\left[n_{a}\left(t_{d}\right)\right]\right. \\
& \left.\cdot \operatorname{Re}\left[n_{b}\left(t_{e}\right)\right] \operatorname{Im}\left[n_{c}\left(t_{e}\right)\right]\right]-E\left[\operatorname{Im}\left[n_{a}\left(t_{d}\right)\right]\right. \\
& \left.\cdot \operatorname{Im}\left[n_{b}\left(t_{e}\right)\right] \operatorname{Re}\left[n_{c}\left(t_{e}\right)\right]\right]-j E\left[\operatorname{Im}\left[n_{a}\left(t_{d}\right)\right]\right. \\
& \left.\cdot \operatorname{Im}\left[n_{b}\left(t_{e}\right)\right] \operatorname{Im}\left[n_{c}\left(t_{e}\right)\right]\right] .
\end{aligned}
$$

In a similar way to get (N.4), we get

$E\left[n_{a}^{*}\left(t_{d}\right) n_{b}^{*}\left(t_{e}\right) n_{c}\left(t_{e}\right)\right]=0$ for Cases $1-10$

\section{O. Fourth-Order Central Moment of Zero-Mean Complex Gaussian Random Variable with Variance $\sigma^{2}$}

$$
\begin{aligned}
E & {\left[n_{l}\left(t_{i}\right) n_{k}^{*}\left(t_{i}\right) n_{l^{\prime}}^{*}\left(t_{i^{\prime}}\right) n_{k^{\prime}}\left(t_{i^{\prime}}\right)\right] } \\
& =E\left[\left(\operatorname{Re}\left[n_{l}\left(t_{i}\right)\right]+j \operatorname{Im}\left[n_{l}\left(t_{i}\right)\right]\right)\right. \\
& \cdot\left(\operatorname{Re}\left[n_{k}\left(t_{i}\right)\right]-j \operatorname{Im}\left[n_{k}\left(t_{i}\right)\right]\right) \\
& \cdot\left(\operatorname{Re}\left[n_{l^{\prime}}\left(t_{i^{\prime}}\right)\right]-j \operatorname{Im}\left[n_{l^{\prime}}\left(t_{i^{\prime}}\right)\right]\right) \\
& \left.\cdot\left(\operatorname{Re}\left[n_{k^{\prime}}\left(t_{i^{\prime}}\right)\right]+j \operatorname{Im}\left[n_{k^{\prime}}\left(t_{i^{\prime}}\right)\right]\right)\right] .
\end{aligned}
$$

(N.6) 
(a) $i=i^{\prime}$.

For $i=i^{\prime},(\mathrm{O} .1)$ can be written as

$$
\begin{aligned}
E & {\left[n_{l}\left(t_{i}\right) n_{k}^{*}\left(t_{i}\right) n_{l^{\prime}}^{*}\left(t_{i}\right) n_{k^{\prime}}\left(t_{i}\right)\right]=E\left[\operatorname{Re}\left[n_{l}\left(t_{i}\right)\right]\right.} \\
& \left.\cdot \operatorname{Re}\left[n_{k}\left(t_{i}\right)\right] \operatorname{Re}\left[n_{l^{\prime}}\left(t_{i}\right)\right] \operatorname{Re}\left[n_{k^{\prime}}\left(t_{i}\right)\right]\right] \\
& +E\left[\operatorname{Re}\left[n_{l}\left(t_{i}\right)\right] \operatorname{Re}\left[n_{k}\left(t_{i}\right)\right]\right] E\left[\operatorname{Im}\left[n_{l^{\prime}}\left(t_{i}\right)\right]\right. \\
\cdot & \left.\operatorname{Im}\left[n_{k^{\prime}}\left(t_{i}\right)\right]\right]-E\left[\operatorname{Re}\left[n_{l}\left(t_{i}\right)\right] \operatorname{Re}\left[n_{k^{\prime}}\left(t_{i}\right)\right]\right] \\
\cdot & E\left[\operatorname{Im}\left[n_{k}\left(t_{i}\right)\right] \operatorname{Im}\left[n_{l^{\prime}}\left(t_{i}\right)\right]\right]+E\left[\operatorname{Im}\left[n_{l}\left(t_{i}\right)\right]\right. \\
& \left.\cdot \operatorname{Im}\left[n_{k}\left(t_{i}\right)\right]\right] E\left[\operatorname{Re}\left[n_{l^{\prime}}\left(t_{i}\right)\right] \operatorname{Re}\left[n_{k^{\prime}}\left(t_{i}\right)\right]\right]
\end{aligned}
$$

$+E\left[\operatorname{Re}\left[n_{k}\left(t_{i}\right)\right] \operatorname{Re}\left[n_{k^{\prime}}\left(t_{i}\right)\right]\right] E\left[\operatorname{Im}\left[n_{l}\left(t_{i}\right)\right]\right.$

$\left.\cdot \operatorname{Im}\left[n_{l^{\prime}}\left(t_{i}\right)\right]\right]-E\left[\operatorname{Re}\left[n_{k}\left(t_{i}\right)\right] \operatorname{Re}\left[n_{l^{\prime}}\left(t_{i}\right)\right]\right]$

$\cdot E\left[\operatorname{Im}\left[n_{l}\left(t_{i}\right)\right] \operatorname{Im}\left[n_{k^{\prime}}\left(t_{i}\right)\right]\right]+E\left[\operatorname{Re}\left[n_{l}\left(t_{i}\right)\right]\right.$

$\left.\cdot \operatorname{Re}\left[n_{l^{\prime}}\left(t_{i}\right)\right]\right] E\left[\operatorname{Im}\left[n_{k}\left(t_{i}\right)\right] \operatorname{Im}\left[n_{k^{\prime}}\left(t_{i}\right)\right]\right]$

$+E\left[\operatorname{Im}\left[n_{l}\left(t_{i}\right)\right] \operatorname{Im}\left[n_{k}\left(t_{i}\right)\right] \operatorname{Im}\left[n_{l^{\prime}}\left(t_{i}\right)\right]\right.$

$\left.\cdot \operatorname{Im}\left[n_{k^{\prime}}\left(t_{i}\right)\right]\right]$.

$E\left[\operatorname{Re}\left[n_{l}\left(t_{i}\right)\right] \operatorname{Re}\left[n_{k}\left(t_{i}\right)\right] \operatorname{Re}\left[n_{l^{\prime}}\left(t_{i}\right)\right] \operatorname{Re}\left[n_{k^{\prime}}\left(t_{i}\right)\right]\right]$

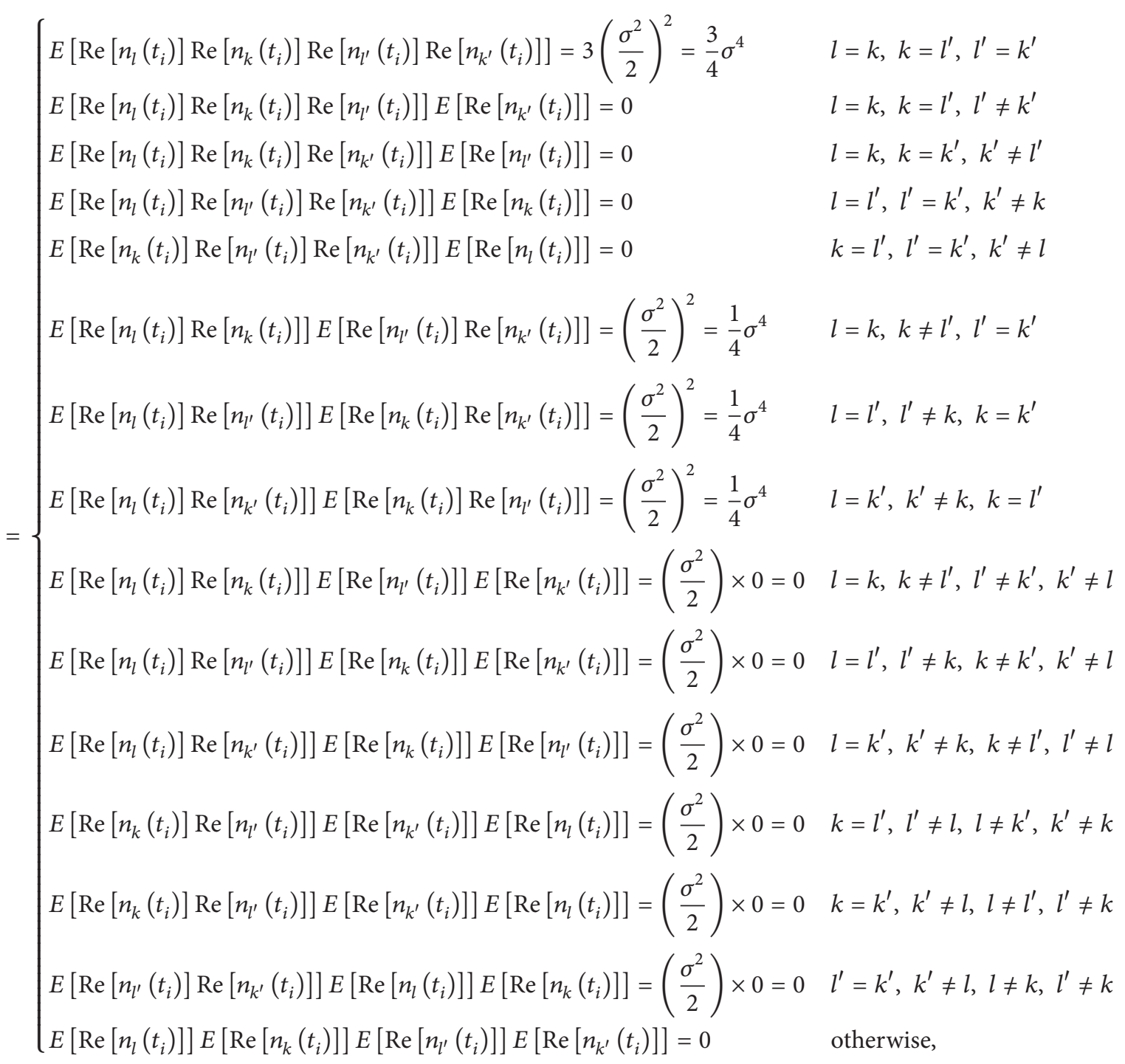


$E\left[\operatorname{Re}\left[n_{l}\left(t_{i}\right)\right] \operatorname{Re}\left[n_{k}\left(t_{i}\right)\right]\right] E\left[\operatorname{Im}\left[n_{l^{\prime}}\left(t_{i}\right)\right] \operatorname{Im}\left[n_{k^{\prime}}\left(t_{i}\right)\right]\right]$

$$
= \begin{cases}E\left[\operatorname{Re}\left[n_{l}\left(t_{i}\right)\right] \operatorname{Re}\left[n_{k}\left(t_{i}\right)\right]\right] E\left[\operatorname{Im}\left[n_{l^{\prime}}\left(t_{i}\right)\right] \operatorname{Im}\left[n_{k^{\prime}}\left(t_{i}\right)\right]\right]=\frac{1}{4} \sigma^{4} & l=k, k=l^{\prime}, l^{\prime}=k^{\prime} \\ E\left[\operatorname{Re}\left[n_{l}\left(t_{i}\right)\right] \operatorname{Re}\left[n_{k}\left(t_{i}\right)\right]\right] E\left[\operatorname{Im}\left[n_{l^{\prime}}\left(t_{i}\right)\right] \operatorname{Im}\left[n_{k^{\prime}}\left(t_{i}\right)\right]\right]=\frac{1}{4} \sigma^{4} & l=k, k \neq l^{\prime}, l^{\prime}=k^{\prime} \\ E\left[\operatorname{Re}\left[n_{l}\left(t_{i}\right)\right] \operatorname{Re}\left[n_{k}\left(t_{i}\right)\right]\right] E\left[\operatorname{Im}\left[n_{l^{\prime}}\left(t_{i}\right)\right] \operatorname{Im}\left[n_{k^{\prime}}\left(t_{i}\right)\right]\right]=0 & \text { otherwise, }\end{cases}
$$

$E\left[\operatorname{Re}\left[n_{l}\left(t_{i}\right)\right] \operatorname{Re}\left[n_{k^{\prime}}\left(t_{i}\right)\right]\right] E\left[\operatorname{Im}\left[n_{k}\left(t_{i}\right)\right] \operatorname{Im}\left[n_{l^{\prime}}\left(t_{i}\right)\right]\right]$

$$
= \begin{cases}E\left[\operatorname{Re}\left[n_{l}\left(t_{i}\right)\right] \operatorname{Re}\left[n_{k^{\prime}}\left(t_{i}\right)\right]\right] E\left[\operatorname{Im}\left[n_{k}\left(t_{i}\right)\right] \operatorname{Im}\left[n_{l^{\prime}}\left(t_{i}\right)\right]\right]=\frac{1}{4} \sigma^{4} & l=k, k=l^{\prime}, l^{\prime}=k^{\prime} \\ E\left[\operatorname{Re}\left[n_{l}\left(t_{i}\right)\right] \operatorname{Re}\left[n_{k^{\prime}}\left(t_{i}\right)\right]\right] E\left[\operatorname{Im}\left[n_{k}\left(t_{i}\right)\right] \operatorname{Im}\left[n_{l^{\prime}}\left(t_{i}\right)\right]\right]=\frac{1}{4} \sigma^{4} & l=k^{\prime}, k^{\prime} \neq k, k=l^{\prime} \\ E\left[\operatorname{Re}\left[n_{l}\left(t_{i}\right)\right] \operatorname{Re}\left[n_{k^{\prime}}\left(t_{i}\right)\right]\right] E\left[\operatorname{Im}\left[n_{k}\left(t_{i}\right)\right] \operatorname{Im}\left[n_{l^{\prime}}\left(t_{i}\right)\right]\right]=0 & \text { otherwise, }\end{cases}
$$

$E\left[\operatorname{Im}\left[n_{l}\left(t_{i}\right)\right] \operatorname{Im}\left[n_{k}\left(t_{i}\right)\right]\right] E\left[\operatorname{Re}\left[n_{l^{\prime}}\left(t_{i}\right)\right] \operatorname{Re}\left[n_{k^{\prime}}\left(t_{i}\right)\right]\right]$

$$
= \begin{cases}E\left[\operatorname{Im}\left[n_{l}\left(t_{i}\right)\right] \operatorname{Im}\left[n_{k}\left(t_{i}\right)\right]\right] E\left[\operatorname{Re}\left[n_{l^{\prime}}\left(t_{i}\right)\right] \operatorname{Re}\left[n_{k^{\prime}}\left(t_{i}\right)\right]\right]=\frac{1}{4} \sigma^{4} & l=k, k=l^{\prime}, l^{\prime}=k^{\prime} \\ E\left[\operatorname{Im}\left[n_{l}\left(t_{i}\right)\right] \operatorname{Im}\left[n_{k}\left(t_{i}\right)\right]\right] E\left[\operatorname{Re}\left[n_{l^{\prime}}\left(t_{i}\right)\right] \operatorname{Re}\left[n_{k^{\prime}}\left(t_{i}\right)\right]\right]=\frac{1}{4} \sigma^{4} & l=k, k \neq l^{\prime}, l^{\prime}=k^{\prime} \\ E\left[\operatorname{Im}\left[n_{l}\left(t_{i}\right)\right] \operatorname{Im}\left[n_{k}\left(t_{i}\right)\right]\right] E\left[\operatorname{Re}\left[n_{l^{\prime}}\left(t_{i}\right)\right] \operatorname{Re}\left[n_{k^{\prime}}\left(t_{i}\right)\right]\right]=0 & \text { otherwise, }\end{cases}
$$

$E\left[\operatorname{Re}\left[n_{k}\left(t_{i}\right)\right] \operatorname{Re}\left[n_{k^{\prime}}\left(t_{i}\right)\right]\right] E\left[\operatorname{Im}\left[n_{l}\left(t_{i}\right)\right] \operatorname{Im}\left[n_{l^{\prime}}\left(t_{i}\right)\right]\right]$

$$
= \begin{cases}E\left[\operatorname{Re}\left[n_{k}\left(t_{i}\right)\right] \operatorname{Re}\left[n_{k^{\prime}}\left(t_{i}\right)\right]\right] E\left[\operatorname{Im}\left[n_{l}\left(t_{i}\right)\right] \operatorname{Im}\left[n_{l^{\prime}}\left(t_{i}\right)\right]\right]=\frac{1}{4} \sigma^{4} & l=k, k=l^{\prime}, l^{\prime}=k^{\prime} \\ E\left[\operatorname{Re}\left[n_{k}\left(t_{i}\right)\right] \operatorname{Re}\left[n_{k^{\prime}}\left(t_{i}\right)\right]\right] E\left[\operatorname{Im}\left[n_{l}\left(t_{i}\right)\right] \operatorname{Im}\left[n_{l^{\prime}}\left(t_{i}\right)\right]\right]=\frac{1}{4} \sigma^{4} & l=l^{\prime}, l^{\prime} \neq k, k=k^{\prime} \\ E\left[\operatorname{Re}\left[n_{k}\left(t_{i}\right)\right] \operatorname{Re}\left[n_{k^{\prime}}\left(t_{i}\right)\right]\right] E\left[\operatorname{Im}\left[n_{l}\left(t_{i}\right)\right] \operatorname{Im}\left[n_{l^{\prime}}\left(t_{i}\right)\right]\right]=0 & \text { otherwise, }\end{cases}
$$

$E\left[\operatorname{Re}\left[n_{k}\left(t_{i}\right)\right] \operatorname{Re}\left[n_{l^{\prime}}\left(t_{i}\right)\right]\right] E\left[\operatorname{Im}\left[n_{l}\left(t_{i}\right)\right] \operatorname{Im}\left[n_{k^{\prime}}\left(t_{i}\right)\right]\right]$

$$
= \begin{cases}E\left[\operatorname{Re}\left[n_{k}\left(t_{i}\right)\right] \operatorname{Re}\left[n_{l^{\prime}}\left(t_{i}\right)\right]\right] E\left[\operatorname{Im}\left[n_{l}\left(t_{i}\right)\right] \operatorname{Im}\left[n_{k^{\prime}}\left(t_{i}\right)\right]\right]=\frac{1}{4} \sigma^{4} & l=k, k=l^{\prime}, l^{\prime}=k^{\prime} \\ E\left[\operatorname{Re}\left[n_{k}\left(t_{i}\right)\right] \operatorname{Re}\left[n_{l^{\prime}}\left(t_{i}\right)\right]\right] E\left[\operatorname{Im}\left[n_{l}\left(t_{i}\right)\right] \operatorname{Im}\left[n_{k^{\prime}}\left(t_{i}\right)\right]\right]=\frac{1}{4} \sigma^{4} & l=k^{\prime}, k^{\prime} \neq k, k=l^{\prime} \\ E\left[\operatorname{Re}\left[n_{k}\left(t_{i}\right)\right] \operatorname{Re}\left[n_{l^{\prime}}\left(t_{i}\right)\right]\right] E\left[\operatorname{Im}\left[n_{l}\left(t_{i}\right)\right] \operatorname{Im}\left[n_{k^{\prime}}\left(t_{i}\right)\right]\right]=0 & \text { otherwise, }\end{cases}
$$

$E\left[\operatorname{Re}\left[n_{l}\left(t_{i}\right)\right] \operatorname{Re}\left[n_{l^{\prime}}\left(t_{i}\right)\right]\right] E\left[\operatorname{Im}\left[n_{k}\left(t_{i}\right)\right] \operatorname{Im}\left[n_{k^{\prime}}\left(t_{i}\right)\right]\right]$

$$
= \begin{cases}E\left[\operatorname{Re}\left[n_{l}\left(t_{i}\right)\right] \operatorname{Re}\left[n_{l^{\prime}}\left(t_{i}\right)\right]\right] E\left[\operatorname{Im}\left[n_{k}\left(t_{i}\right)\right] \operatorname{Im}\left[n_{k^{\prime}}\left(t_{i}\right)\right]\right]=\frac{1}{4} \sigma^{4} & l=k, k=l^{\prime}, l^{\prime}=k^{\prime} \\ E\left[\operatorname{Re}\left[n_{l}\left(t_{i}\right)\right] \operatorname{Re}\left[n_{l^{\prime}}\left(t_{i}\right)\right]\right] E\left[\operatorname{Im}\left[n_{k}\left(t_{i}\right)\right] \operatorname{Im}\left[n_{k^{\prime}}\left(t_{i}\right)\right]\right]=\frac{1}{4} \sigma^{4} & l=l^{\prime}, l^{\prime} \neq k, k=k^{\prime} \\ E\left[\operatorname{Re}\left[n_{l}\left(t_{i}\right)\right] \operatorname{Re}\left[n_{l^{\prime}}\left(t_{i}\right)\right]\right] E\left[\operatorname{Im}\left[n_{k}\left(t_{i}\right)\right] \operatorname{Im}\left[n_{k^{\prime}}\left(t_{i}\right)\right]\right]=0 & \text { otherwise, }\end{cases}
$$

$E\left[\operatorname{Im}\left[n_{l}\left(t_{i}\right)\right] \operatorname{Im}\left[n_{k}\left(t_{i}\right)\right] \operatorname{Im}\left[n_{l^{\prime}}\left(t_{i}\right)\right] \operatorname{Im}\left[n_{k^{\prime}}\left(t_{i}\right)\right]\right]$

$$
= \begin{cases}E\left[\operatorname{Im}\left[n_{l}\left(t_{i}\right)\right] \operatorname{Im}\left[n_{k}\left(t_{i}\right)\right] \operatorname{Im}\left[n_{l^{\prime}}\left(t_{i}\right)\right] \operatorname{Im}\left[n_{k^{\prime}}\left(t_{i}\right)\right]\right]=\frac{3}{4} \sigma^{4} & l=k, k=l^{\prime}, l^{\prime}=k^{\prime} \\ E\left[\operatorname{Im}\left[n_{l}\left(t_{i}\right)\right] \operatorname{Im}\left[n_{k}\left(t_{i}\right)\right]\right] E\left[\operatorname{Im}\left[n_{l^{\prime}}\left(t_{i}\right)\right] \operatorname{Im}\left[n_{k^{\prime}}\left(t_{i}\right)\right]\right]=\frac{1}{4} \sigma^{4} & l=k, k \neq l^{\prime}, l^{\prime}=k^{\prime} \\ E\left[\operatorname{Im}\left[n_{l}\left(t_{i}\right)\right] \operatorname{Im}\left[n_{l^{\prime}}\left(t_{i}\right)\right]\right] E\left[\operatorname{Im}\left[n_{k}\left(t_{i}\right)\right] \operatorname{Im}\left[n_{k^{\prime}}\left(t_{i}\right)\right]\right]=\frac{1}{4} \sigma^{4} & l=l^{\prime}, l^{\prime} \neq k, k=k^{\prime} \\ E\left[\operatorname{Im}\left[n_{l}\left(t_{i}\right)\right] \operatorname{Im}\left[n_{k^{\prime}}\left(t_{i}\right)\right]\right] E\left[\operatorname{Im}\left[n_{k}\left(t_{i}\right)\right] \operatorname{Im}\left[n_{l^{\prime}}\left(t_{i}\right)\right]\right]=\frac{1}{4} \sigma^{4} & l=k^{\prime}, k^{\prime} \neq k, k=l^{\prime} \\ E\left[\operatorname{Im}\left[n_{l}\left(t_{i}\right)\right] \operatorname{Im}\left[n_{k}\left(t_{i}\right)\right] \operatorname{Im}\left[n_{l^{\prime}}\left(t_{i}\right)\right] \operatorname{Im}\left[n_{k^{\prime}}\left(t_{i}\right)\right]\right]=0 & \text { otherwise. }\end{cases}
$$


From (O.3)-(O.10), $E\left[n_{l}\left(t_{i}\right) n_{k}^{*}\left(t_{i}\right) n_{l^{\prime}}^{*}\left(t_{i}\right) n_{k^{\prime}}\left(t_{i}\right)\right]$ in (O.2) is given by

$$
\begin{aligned}
& E\left[n_{l}\left(t_{i}\right) n_{k}^{*}\left(t_{i}\right) n_{l^{\prime}}^{*}\left(t_{i}\right) n_{k^{\prime}}\left(t_{i}\right)\right] \\
& \quad= \begin{cases}\frac{3}{4} \sigma^{4}+\frac{1}{4} \sigma^{4}-\frac{1}{4} \sigma^{4}+\frac{1}{4} \sigma^{4}+\frac{1}{4} \sigma^{4}-\frac{1}{4} \sigma^{4}+\frac{1}{4} \sigma^{4}+\frac{3}{4} \sigma^{4}=2 \sigma^{4} & l=k, k=l^{\prime}, l^{\prime}=k^{\prime} \\
\frac{1}{4} \sigma^{4}+\frac{1}{4} \sigma^{4}+\frac{1}{4} \sigma^{4}+\frac{1}{4} \sigma^{4}=\sigma^{4} & l=k, k \neq l^{\prime}, l^{\prime}=k^{\prime} \\
\frac{1}{4} \sigma^{4}+\frac{1}{4} \sigma^{4}+\frac{1}{4} \sigma^{4}+\frac{1}{4} \sigma^{4}=\sigma^{4} & l=l^{\prime}, l^{\prime} \neq k, k=k^{\prime} \\
0 & \text { otherwise. }\end{cases}
\end{aligned}
$$

(b) $i \neq i^{\prime}$ :

$E\left[n_{l}\left(t_{i}\right) n_{k}^{*}\left(t_{i}\right) n_{l^{\prime}}^{*}\left(t_{i^{\prime}}\right) n_{k^{\prime}}\left(t_{i^{\prime}}\right)\right]=E\left[\operatorname{Re}\left[n_{l}\left(t_{i}\right)\right]\right.$

$\left.\cdot \operatorname{Re}\left[n_{k}\left(t_{i}\right)\right] \operatorname{Re}\left[n_{l^{\prime}}\left(t_{i^{\prime}}\right)\right] \operatorname{Re}\left[n_{k^{\prime}}\left(t_{i^{\prime}}\right)\right]\right]$

$+E\left[\operatorname{Re}\left[n_{l}\left(t_{i}\right)\right] \operatorname{Re}\left[n_{k}\left(t_{i}\right)\right] \operatorname{Im}\left[n_{l^{\prime}}\left(t_{i^{\prime}}\right)\right]\right.$

$\left.\cdot \operatorname{Im}\left[n_{k^{\prime}}\left(t_{i^{\prime}}\right)\right]\right]+E\left[\operatorname{Im}\left[n_{l}\left(t_{i}\right)\right] \operatorname{Im}\left[n_{k}\left(t_{i}\right)\right]\right.$

$\left.\cdot \operatorname{Re}\left[n_{l^{\prime}}\left(t_{i^{\prime}}\right)\right] \operatorname{Re}\left[n_{k^{\prime}}\left(t_{i^{\prime}}\right)\right]\right]+E\left[\operatorname{Im}\left[n_{l}\left(t_{i}\right)\right]\right.$

$\left.\cdot \operatorname{Im}\left[n_{k}\left(t_{\mathrm{i}}\right)\right] \operatorname{Im}\left[n_{l^{\prime}}\left(t_{i^{\prime}}\right)\right] \operatorname{Im}\left[n_{k^{\prime}}\left(t_{i^{\prime}}\right)\right]\right]$.

Using a similar way to get (O.11), for $i \neq i^{\prime}$, $E\left[n_{l}\left(t_{i}\right) n_{k}^{*}\left(t_{i}\right) n_{l^{\prime}}^{*}\left(t_{i^{\prime}}\right) n_{k^{\prime}}\left(t_{i^{\prime}}\right)\right]$ is given by

$$
\begin{aligned}
& E\left[n_{l}\left(t_{i}\right) n_{k}^{*}\left(t_{i}\right) n_{l^{\prime}}^{*}\left(t_{i^{\prime}}\right) n_{k^{\prime}}\left(t_{i^{\prime}}\right)\right] \\
& = \begin{cases}\frac{1}{4} \sigma^{4}+\frac{1}{4} \sigma^{4}+\frac{1}{4} \sigma^{4}+\frac{1}{4} \sigma^{4}=\sigma^{4} & l=k, k=l^{\prime}, l^{\prime}=k^{\prime} \\
\frac{1}{4} \sigma^{4}+\frac{1}{4} \sigma^{4}+\frac{1}{4} \sigma^{4}+\frac{1}{4} \sigma^{4}=\sigma^{4} & l=k, k \neq l^{\prime}, l^{\prime}=k^{\prime} \\
0 & \text { otherwise. }\end{cases}
\end{aligned}
$$

\section{P. Derivation of $E\left[\delta R_{l k} \delta R_{l^{\prime} k^{\prime}}^{*}\right]$}

$$
\begin{aligned}
\widehat{R}_{l k}^{\prime} & =\frac{1}{L} \sum_{i=1}^{L}\left(x_{l}\left(t_{i}\right) x_{k}^{*}\left(t_{i}\right)+x_{l}\left(t_{i}\right) n_{k}^{*}\left(t_{i}\right)\right. \\
& \left.+n_{l}\left(t_{i}\right) x_{k}^{*}\left(t_{i}\right)+n_{l}\left(t_{i}\right) n_{k}^{*}\left(t_{i}\right)\right), \\
\widehat{R}_{l k} & =\frac{1}{L} \sum_{i=1}^{L}\left(x_{l}\left(t_{i}\right) x_{k}^{*}\left(t_{i}\right)\right), \\
\delta R_{l k} & =\frac{1}{L} \sum_{i=1}^{L}\left(x_{l}\left(t_{i}\right) n_{k}^{*}\left(t_{i}\right)+n_{l}\left(t_{i}\right) x_{k}^{*}\left(t_{i}\right)\right. \\
& \left.+n_{l}\left(t_{i}\right) n_{k}^{*}\left(t_{i}\right)\right) .
\end{aligned}
$$

From (P.3), $E\left[\delta R_{l k} \delta R_{l^{\prime} k^{\prime}}^{*}\right]$ is given by

$$
\begin{aligned}
E[ & \left.\delta R_{l k} \delta R_{l^{\prime} k^{\prime}}^{*}\right] \\
& =\frac{1}{L^{2}}\left(\sum _ { i = i _ { i ^ { \prime } = 1 } } ^ { L } \sum _ { l } ^ { L } \left(x_{l}\left(t_{i}\right) x_{l^{\prime}}^{*}\left(t_{i^{\prime}}\right) E\left(n_{k}^{*}\left(t_{i}\right) n_{k^{\prime}}\left(t_{i^{\prime}}\right)\right)\right.\right. \\
& +x_{l}\left(t_{i}\right) x_{k^{\prime}}\left(t_{i^{\prime}}\right) E\left(n_{k}^{*}\left(t_{i}\right) n_{l^{\prime}}^{*}\left(t_{i^{\prime}}\right)\right) \\
& +x_{l}\left(t_{i}\right) E\left(n_{k}^{*}\left(t_{i}\right) n_{l^{\prime}}^{*}\left(t_{i^{\prime}}\right) n_{k^{\prime}}\left(t_{i^{\prime}}\right)\right) \\
& +x_{k}^{*}\left(t_{i}\right) x_{l^{\prime}}^{*}\left(t_{i^{\prime}}\right) E\left(n_{l}\left(t_{i}\right) n_{k^{\prime}}\left(t_{i^{\prime}}\right)\right) \\
& +x_{k}^{*}\left(t_{i}\right) x_{k^{\prime}}\left(t_{i^{\prime}}\right) E\left(n_{l}\left(t_{i}\right) n_{l^{\prime}}^{*}\left(t_{i^{\prime}}\right)\right) \\
& +x_{k}^{*}\left(t_{i}\right) E\left(n_{l}\left(t_{i}\right) n_{l^{\prime}}^{*}\left(t_{i^{\prime}}\right) n_{k^{\prime}}\left(t_{i^{\prime}}\right)\right) \\
& +x_{l^{\prime}}^{*}\left(t_{i^{\prime}}\right) E\left(n_{l}\left(t_{i}\right) n_{k}^{*}\left(t_{i}\right) n_{k^{\prime}}\left(t_{i^{\prime}}\right)\right) \\
& +x_{k^{\prime}}\left(t_{i^{\prime}}\right) E\left(n_{l}\left(t_{i}\right) n_{k}^{*}\left(t_{i}\right) n_{l^{\prime}}^{*}\left(t_{i^{\prime}}\right)\right) \\
& \left.\left.+E\left(n_{l}\left(t_{i}\right) n_{k}^{*}\left(t_{i}\right) n_{l^{\prime}}^{*}\left(t_{i^{\prime}}\right) n_{k^{\prime}}\left(t_{i^{\prime}}\right)\right)\right)\right)
\end{aligned}
$$

where the last equality follows since the noiseless signals are not stochastic.

In Appendices $\mathrm{M}$ and $\mathrm{N}$, it is shown that the followings are identically zero:

$$
\begin{aligned}
E\left(n_{k}\left(t_{i}\right) n_{l^{\prime}}\left(t_{i^{\prime}}\right)\right) & =0, \\
E\left(n_{k}\left(t_{i}\right) n_{l}^{*}\left(t_{i}\right) n_{l^{\prime}}\left(t_{i^{\prime}}\right)\right) & =0, \\
E\left(n_{k}\left(t_{i}\right) n_{k^{\prime}}^{*}\left(t_{i^{\prime}}\right) n_{l^{\prime}}\left(t_{i^{\prime}}\right)\right) & =0, \\
E\left(n_{l}^{*}\left(t_{i}\right) n_{k^{\prime}}^{*}\left(t_{i^{\prime}}\right)\right) & =0, \\
E\left(n_{k}\left(t_{i}\right) n_{l}^{*}\left(t_{i}\right) n_{k^{\prime}}^{*}\left(t_{i^{\prime}}\right)\right) & =0, \\
E\left(n_{l}^{*}\left(t_{i}\right) n_{k^{\prime}}^{*}\left(t_{i^{\prime}}\right) n_{l^{\prime}}\left(t_{i^{\prime}}\right)\right) & =0 .
\end{aligned}
$$


Using (P.5) in (P.4), (P.4) is simplified to $E\left[\delta R_{l k} \delta R_{l^{\prime} k^{\prime}}^{*}\right]$,

$$
\frac{1}{L^{2}}\left(\sum_{i=1}^{L} \sum_{i^{\prime}=1}^{L}\left(x_{l}\left(t_{i}\right) x_{l^{\prime}}^{*}\left(t_{i^{\prime}}\right) E\left(n_{k}^{*}\left(t_{i}\right) n_{k^{\prime}}\left(t_{i^{\prime}}\right)\right)+x_{k}^{*}\left(t_{i}\right) x_{k^{\prime}}\left(t_{i^{\prime}}\right) E\left(n_{l}\left(t_{i}\right) n_{l^{\prime}}^{*}\left(t_{i^{\prime}}\right)\right)+E\left(n_{l}\left(t_{i}\right) n_{k}^{*}\left(t_{i}\right) n_{l^{\prime}}^{*}\left(t_{i^{\prime}}\right) n_{k^{\prime}}\left(t_{i^{\prime}}\right)\right)\right)\right)
$$

where the second moments and the fourth moment in (P.6) are derived in Appendices $\mathrm{M}$ and $\mathrm{O}$, respectively. Finally,

$$
E\left[(\delta R)_{l k}(\delta R)_{l^{\prime} k^{\prime}}^{*}\right] \text { is given by }
$$

$$
E\left[(\delta R)_{l k}(\delta R)_{l^{\prime} k^{\prime}}^{*}\right]= \begin{cases}\frac{1}{L^{2}}\left(\sigma^{2} \sum_{i=1}^{L}\left(x_{l}\left(t_{i}\right) x_{l^{\prime}}^{*}\left(t_{i}\right)+x_{k}^{*}\left(t_{i}\right) x_{k^{\prime}}\left(t_{i}\right)\right)+\left(L^{2}+L\right) \sigma^{4}\right) & \left(l=k, k=l^{\prime}, l^{\prime}=k^{\prime}\right) \\ \frac{1}{L^{2}}\left(\sigma^{2} \sum_{i=1}^{L}\left(x_{k}^{*}\left(t_{i}\right) x_{k^{\prime}}\left(t_{i}\right)\right)\right) & \left(l=k, k=l^{\prime}, l^{\prime} \neq k^{\prime}\right) \\ \frac{1}{L^{2}}\left(\sigma^{2} \sum_{i=1}^{L}\left(x_{l}\left(t_{i}\right) x_{l^{\prime}}^{*}\left(t_{i}\right)\right)\right) & \left(l=l^{\prime}, l^{\prime}=k^{\prime}, k^{\prime} \neq k\right) \\ \frac{1}{L^{2}}\left(\sigma^{2} \sum_{i=1}^{L}\left(x_{k}^{*}\left(t_{i}\right) x_{k^{\prime}}\left(t_{i}\right)\right)\right) & \left(l=k^{\prime}, k^{\prime}=l^{\prime}, l^{\prime} \neq k\right) \\ \frac{1}{L^{2}}\left(\sigma^{2} \sum_{i=1}^{L}\left(x_{l}\left(t_{i}\right) x_{l^{\prime}}^{*}\left(t_{i}\right)\right)\right) & \left(l=k, k=k^{\prime}, k^{\prime} \neq l^{\prime}\right) \\ \frac{1}{L^{2}}\left(\sigma^{4}\right) & \left(l=k, k \neq l^{\prime}, l^{\prime}=k^{\prime}\right) \\ \frac{1}{L^{2}}\left(\sigma^{2} \sum_{i=1}^{L}\left(x_{l}\left(t_{i}\right) x_{l^{\prime}}^{*}\left(t_{i}\right)+x_{k}^{*}\left(t_{i}\right) x_{k^{\prime}}\left(t_{i}\right)\right)+L \sigma^{4}\right) \\ \frac{1}{L^{2}}\left(\sigma^{2} \sum_{i=1}^{L}\left(x_{k}^{*}\left(t_{i}\right) x_{k^{\prime}}\left(t_{i}\right)\right)\right) \\ \frac{1}{L^{2}}\left(\sigma^{2} \sum_{i=1}^{L}\left(x_{l}\left(t_{i}\right) x_{l^{\prime}}^{*}\left(t_{i}\right)\right)\right) & \left(l=l^{\prime}, l^{\prime} \neq k, k=k^{\prime}\right) \\ & \left(l=l^{\prime}, l^{\prime} \neq k, k \neq k^{\prime}\right) \\ & \left(k=k^{\prime}, k^{\prime} \neq l, l \neq l^{\prime}\right) .\end{cases}
$$

\section{Competing Interests}

The authors declare that they have no competing interests.

\section{References}

[1] Z.-M. Liu, Z.-T. Huang, Y.-Y. Zhou, and J. Liu, "Direction-ofarrival estimation of noncircular signals via sparse representation," IEEE Transactions on Aerospace and Electronic Systems, vol. 48, no. 3, pp. 2690-2698, 2012.

[2] B. Liao and S.-C. Chan, "Direction-of-arrival estimation in subarrays-based linear sparse arrays with gain/phase uncertainties," IEEE Transactions on Aerospace and Electronic Systems, vol. 49, no. 4, pp. 2268-2280, 2013.
[3] T. N. Ferreira, S. L. Netto, and P. S. R. Diniz, "Directionof-arrival estimation using a low-complexity covariance-based approach," IEEE Transactions on Aerospace and Electronic Systems, vol. 48, no. 3, pp. 1924-1934, 2012.

[4] Y. Song, "Decoupled 2D direction-of-arrival estimation using partly calibrated vector hydrophones," IEEE Transactions on Aerospace and Electronic Systems, vol. 49, no. 4, pp. 2470-2477, 2013.

[5] Y. Song and K. T. Wong, "A lower bound of direction-ofarrival estimation for an acoustic vector sensor subject to sensor breakdown," IEEE Transactions on Aerospace and Electronic Systems, vol. 48, no. 4, pp. 3703-3708, 2012.

[6] P. Stoica and A. Nehorai, "MUSIC, maximum likelihood, and Cramer-Rao bound," IEEE Transactions on Acoustics, Speech, and Signal Processing, vol. 37, no. 5, pp. 720-741, 1989. 
[7] P. Stoica and A. Nehorai, "MUSIC, maximum likelihood, and Cramer-Rao bound: further results and comparisons," IEEE Transactions on Acoustics, Speech, and Signal Processing, vol. 38, no. 12, pp. 2140-2150, 1990.

[8] P. Stoica and A. Nehorai, "Performance study of conditional and unconditional direction-of-arrival estimation," IEEE Transactions on Acoustics, Speech, and Signal Processing, vol. 38, no. 10, pp. 1783-1795, 1990.

[9] B. G. Quinn and P. J. Kootsookos, "Threshold behavior of the maximum likelihood estimator of frequency," IEEE Transactions on Signal Processing, vol. 42, no. 11, pp. 3291-3294, 1994.

[10] F. Athley, "Threshold region performance of maximum likelihood direction of arrival estimators," IEEE Transactions on Signal Processing, vol. 53, no. 4, pp. 1359-1373, 2005.

[11] P. Forster, P. Larzabal, and E. Boyer, "Threshold performance analysis of maximum likelihood DOA estimation," IEEE Transactions on Signal Processing, vol. 52, no. 11, pp. 3183-3191, 2004.

[12] F. Athley, "Performance analysis of DOA estimation in the threshold region," in Proceedings of the 2002 IEEE International Conference on Acoustic, Speech, and Signal Processing (ICASSP '02), vol. 3, pp. 3017-3020, Orlando, Fla, USA, May 2002.

[13] B. Ottersten, M. Viberg, P. Stoica, and A. Nehorai, "Exact and large sample maximum likelihood techniques for parameter estimation and detection in array processing," in Radar Array Processing, vol. 25 of Springer Series in Information Sciences, pp. 99-151, Springer, Berlin, Germany, 1993.

[14] H. C. So, Y. T. Chan, K. C. Ho, and Y. Chen, "Simple formulae for bias and mean square error computation [DSP Tips and Tricks]," IEEE Signal Processing Magazine, vol. 30, no. 4, pp. 162165, 2013.

[15] H. Anton and C. Rorres, Elementary Linear Algebra, John Wiley \& Sons, New York, NY, USA, 10th edition, 2011.

[16] Z. Chen, G. Gokeda, and Y. Yu, Introduction to Direction-ofArrival Estimation, Artech House, 2010. 


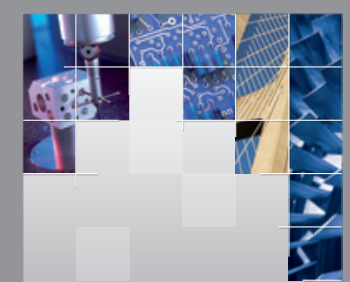

\section{Enfincering}
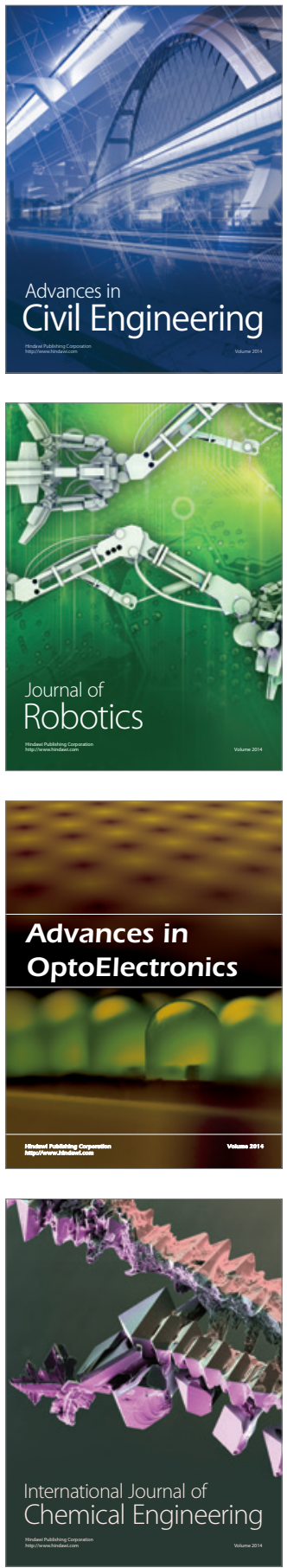

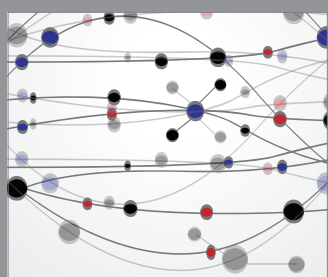

The Scientific World Journal

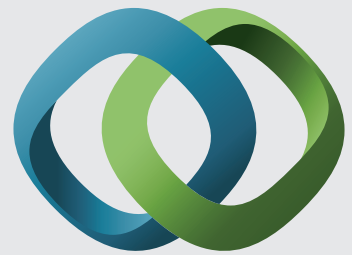

\section{Hindawi}

Submit your manuscripts at

https://www.hindawi.com
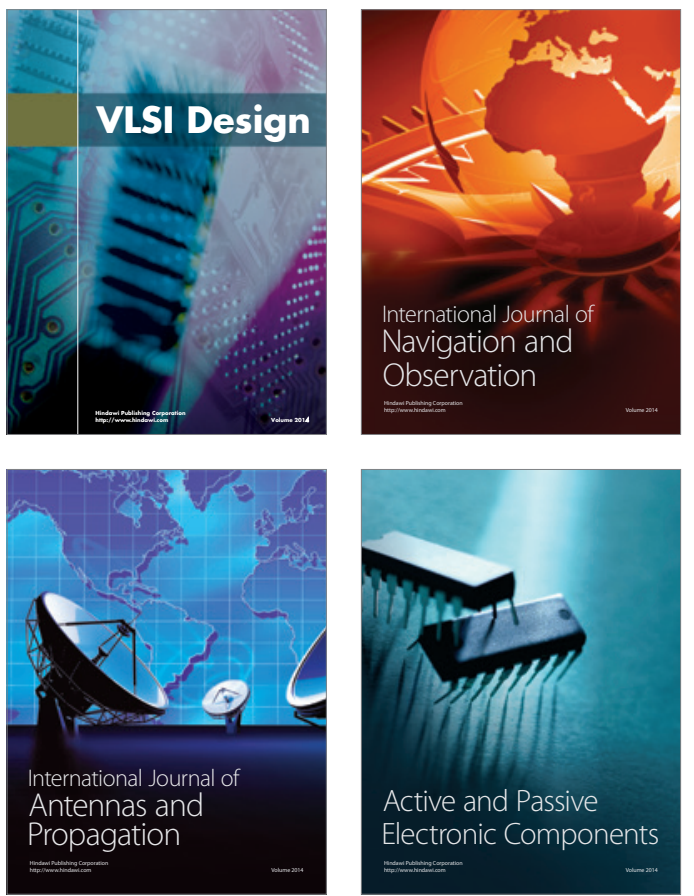
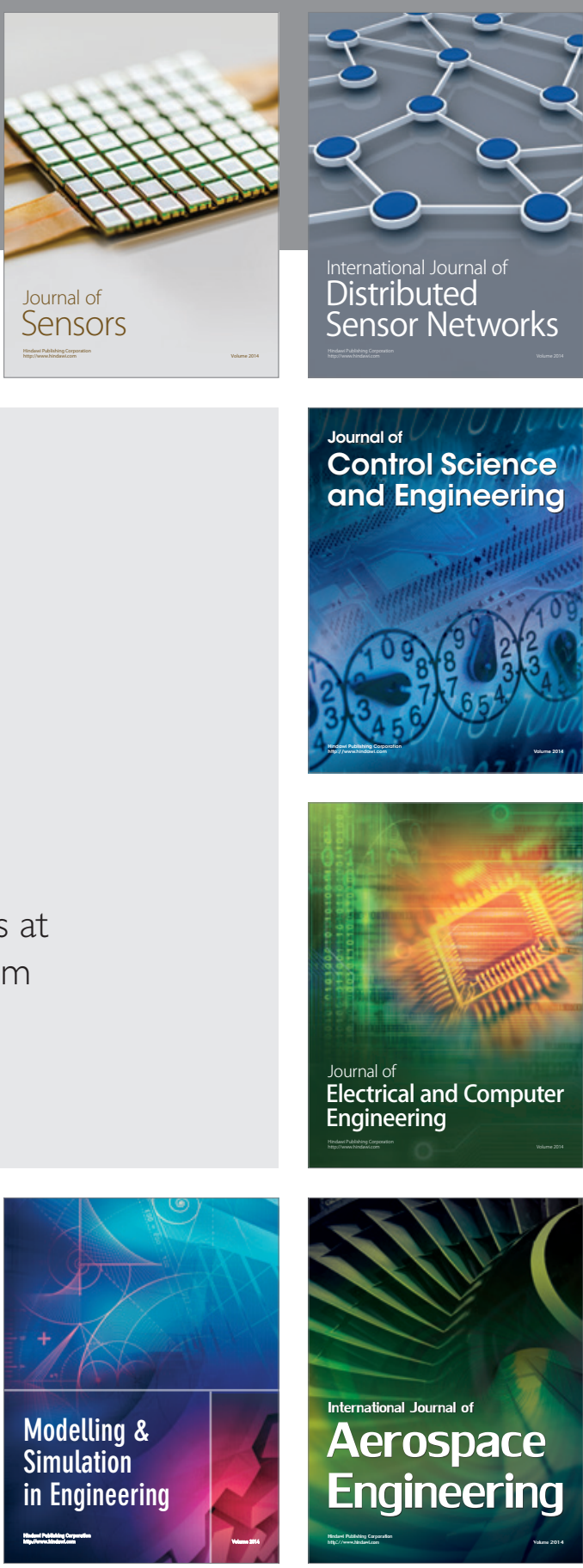

International Journal of

Distributed

Sensor Networks

$-$

Joumal of

Control Science

and Engineering
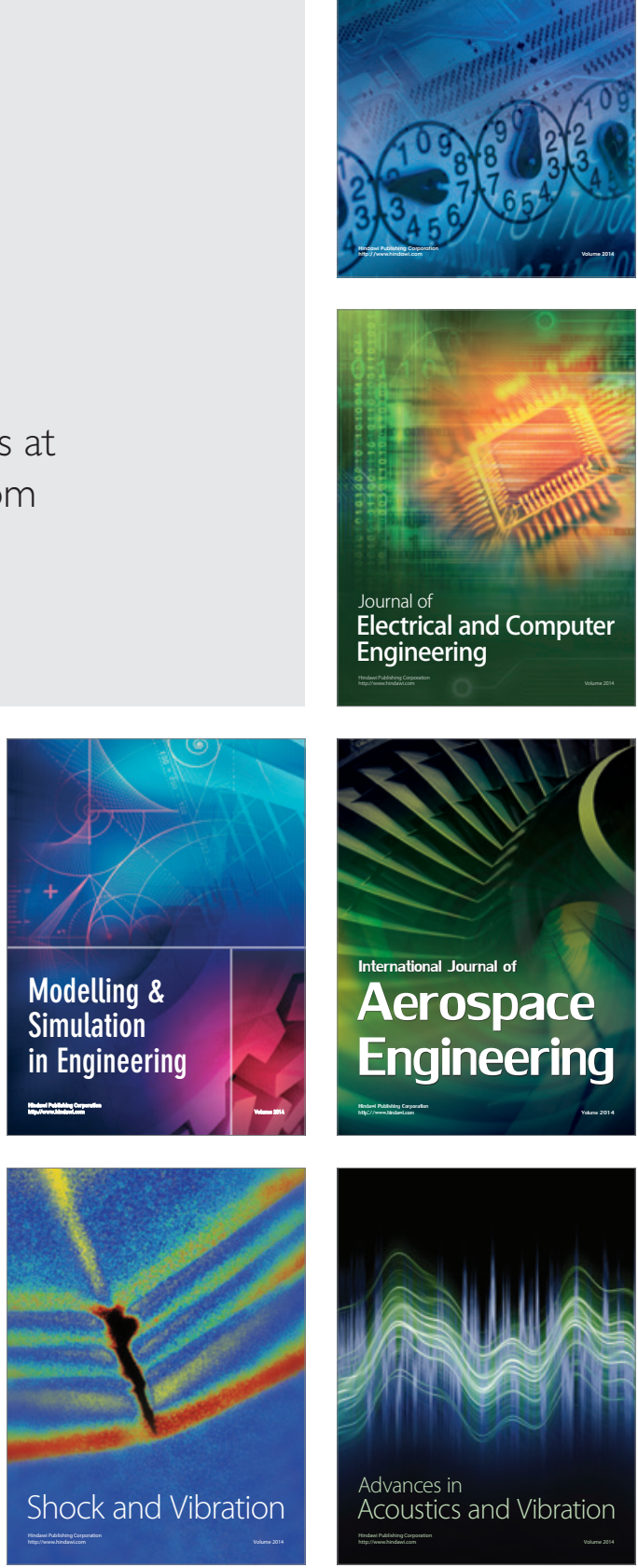